\title{
A Simplified Proof of Desingularization and Applications
}

\section{Ana María Bravo, Santiago Encinas and Orlando Villamayor U.}

\begin{abstract}
This paper contains a short and simplified proof of desingularization over fields of characteristic zero, together with various applications to other problems in algebraic geometry (among others, the study of the behavior of desingularization of families of embedded schemes, and a formulation of desingularization which is stronger than Hironaka's). Our proof avoids the use of the Hilbert-Samuel function and Hironaka's notion of normal flatness: First we define a procedure for principalization of ideals (i. e. a procedure to make an ideal invertible), and then we show that desingularization of a closed subscheme $X$ is achieved by using the procedure of principalization for the ideal $\mathcal{I}(X)$ associated to the embedded scheme $X$. The paper intends to be an introduction to the subject, focused on the motivation of ideas used in this new approach, and particularly on applications, some of which do not follow from Hironaka's proof.
\end{abstract}

\section{Part I. Introduction}

\section{Introduction}

We present a unified proof, for the case of schemes over a field of characteristic zero and of compact analytic spaces, of two central theorems in algebraic geometry: The resolution of singularities of schemes, and the principalization of ideals (see Section 2 for precise statements of our results). The existence of resolution of singularities is one of the most important results in the area, due to its large number of applications, while principalization of ideals is related to the classical problem of elimination of base points of a linear system.

2000 Mathematics Subject Classification: $14 \mathrm{E} 15$.

Keywords: Resolution of singularities, desingularization. 
Resolution of singularities of schemes. Given a reduced scheme $X$ of finite type over a field of characteristic zero, find a proper and birational morphism

$$
\pi: X^{\prime} \rightarrow X
$$

such that $X^{\prime}$ is non-singular and $\pi$ induces an isomorphism over the regular points of $X$.

Principalization. Given a non zero sheaf of ideals $J$ in non-singular variety $W$, find a proper and birational morphism,

$$
\pi: W^{\prime} \rightarrow W
$$

such that $W^{\prime}$ is non-singular and $J_{W^{\prime}}$ is locally principal.

The purpose of this paper is threefold: We present a simplified proof of both theorems, already indicated in [18], see also [19] (this is done in Parts II and V of this manuscript); we summarize some applications of our approach, together with new results (see Part III); and finally, in Part IV, we motivate the main ideas behind the invariants that we use in this proof of desingularization (this part is purely expository). Our very short proof of desingularization presented in 5.8, makes use of the algorithm developed essentially in Part V (and recently implemented in MAPLE). This provides a proof of desingularization that does not exceed thirty pages.

What makes this work different from other approaches to desingularization?

In his monumental work ([23]), Hironaka gave an existential proof of resolution of singularities, and, in so doing, he also provided an existential proof for principalization of ideals. Hironaka's approach is based in two main ideas: Reduction to the hypersurface case, and induction on the dimension. As for the reduction to the hypersurface case, he shows that given a variety $X$, it is possible to describe it as an intersection of a finite number of hypersurfaces $\left\{H_{i}\right\}_{i \in I}$, in such a way that the intersection of the worst singularities of the hypersurfaces $\left\{H_{i}\right\}_{i \in I}$ is the set of the worst singularities of $X$. Then he proves, using an existential argument, that there is a sequence of blowing-ups such that the singularities of the hypersurfaces are better, and hence the singularities of $X$ are better too.

To find the hypersurfaces $H_{i}$, Hironaka considers a stratification of $X$ by means of the Hilbert-Samuel function. The worst singularities of $X$ correspond to the stratum of points where this function is maximum. He then provides an existential argument to show that by blowing-up at smooth centers contained in the maximum stratum of the Hilbert-Samuel function, the maximum drops (the containment condition over the centers is usually referred 
as normal flatness). Once this maximum drops, new hypersurfaces $H_{i}$ have to be chosen. All this requires the use of standard bases, algorithms of division and the notion of strict transform of ideals (see [26], [32] and [20] for more information on Hironaka's line of proof, and also Section 7, where the notion of strict transform of an ideal is defined).

During the eighties and nineties, the first constructive proofs of resolution of singularities appeared: [7], [34] (and [35]), [17] (see also [8]). However, all of them were based on Hironaka's approach. These proofs provided a general algorithm of desingularization indicating where to blow-up in order to eliminate the singularities in a step by step procedure. The idea is to define invariants of singular points, and to show that these invariants improve when blowing up the set of worst points. Embedded desingularization is then achieved by repeatedly blowing up the set of worst points. All algorithmic procedures mentioned above, make use, as Hironaka did in his original work, of the Hilbert Samuel function and the strict transform of ideals. From an algorithmic point of view, the notion of strict transform of an ideal is quite complicated, except in the case of a hypersurface.

What makes this present proof different from the previous ones is that we show that embedded desingularization can be achieved avoiding the use of Hilbert Samuel functions and the control of strict transforms of ideals. Instead of using a standard basis we take any set of generators of an ideal, and we do not need to change these generators in the procedure of desingularization.

Our strategy is based in the fact that embedded desingularization turns out to be a straightforward corollary of a simpler result: The Algorithmic Resolution of Basic Objects. While related to Hironaka's idealistic exponents (see [24]), the notion of basic object is different and easier to work with. Our approach follows from the study of local properties of the algorithmic resolution process for basic objects developed in [35] and [18] and also included in this paper. In fact the algorithm of desingularization in [35] was also obtained by a suitable application of this same algorithm of resolution of basic objects but in a different manner, and our proof of equivariance (compatibility with group actions) is also similar to the proof in that paper. Furthermore, our new proof coincides with that from [35] in the hypersurface case, so explicit examples for algorithmic desingularization of hypersurfaces can be found in [35, Section 8] and in [36] (Hilbert-Samuel functions were not required in Hironaka's proof in the hypersurface case).

Among other applications, this algorithm of resolution of basic objects has been implemented as a computer program by G. Bodnár and J. Schicho. As a consequence these authors have developed a program which resolves singularities of hypersurfaces (see [10] and [11]). The proof of resolution 
of singularities that we give in 5.8 shows that that this implemented algorithm of resolution of basic objects, as it stands, also applies for embedded desingularization of any scheme, even if not a hypersurface (see Section 11).

This new approach to resolution of singularities also allows us to go beyond Hironaka's desingularization: Based on the ideas developed here, in [13] we present a stronger formulation of desingularization (see Section 7 for a precise statement of this result, as well as additional applications). See also Part III for more applications.

Finally, there are other results in desingularization within this new framework and related to this one: For instance, Matsuki's lucid presentation of the ideas behind the algorithms of desingularization in [28], which is based in the work developed in [19]; or [15], where an algorithm of principalization and of desingularization is stated from a different point of view, using a new presentation of the local invariant.

We also should mention the papers [2], [3] and [12], where a completely different proof of resolution of singularities it is given, leading to the so called weak desingularization of varieties. As opposed to the algorithms mentioned before, in the weak desingularization approach regular points of the variety may be modified in the desingularization process. More generally, see [22] for an interesting introduction to the problem of desingularization.

As indicated above, this manuscript is a self contained presentation which intends to provide an introduction to the subject, particularly focused in the motivation of the main ideas, and hopefully a reference for future applications. To this end we have organized the paper so that the reader can easily get into the statement of the main results and some of their applications without having to go through the technicalities of the proofs.

More precisely, the paper is organized as follows: In the next section, the last of Part I, we state the theorems of resolution of singularities and principalization of ideals that we are going to prove.

Part II is devoted to presenting the theory of basic objects: This notion is introduced in Section 3, and those of equivariance, and of algorithmic resolution of basic objects, in Sections 4 and 5. After presenting the properties of this implemented algorithm of resolution of basic objects, in 5.8 we give a short proof of desingularization for equidimensional schemes (in [13] we provide a proof which works in the general case). In Section 6 we introduce the two main equivariant functions involved in the construction of the algorithms of resolution.

In Part III we present applications of our results: In Section 7 the notions of weak and strict transforms of ideals are discussed, and a stronger version of Hironaka's Theorem is stated; in Section 8 we discuss the extension of our results to a wider class of schemes (which are not necessarily of finite 
type over a field); Sections 9 and 10 deal respectively with resolution of locally embedded varieties and resolution in families of varieties; finally, in Section 11 there are some indications on how the algorithm of desingularization can be implemented as a computer program which works for arbitrary varieties (and not only in the hypersurface case).

Part IV is devoted to giving a flavor of how the algorithm of resolution of basic objects works. The algorithm is described in Part V.

Acknowledgments. Part of the material contained in this manuscript has been presented in a series of seminars at the University of Purdue, during the Spring in 2001. We profited from discussions with Gabrielov, Matsuki, Moh and Nobile.

\section{Formulation of the Theorems}

The main point in the statement of the problems of resolution of singularities and principalization of ideals is the construction of a birational morphism of regular schemes $\pi: W^{\prime} \rightarrow W$. This morphism will be defined as a composition of monoidal transformations at closed and regular centers. If $e_{1}: W_{1} \longrightarrow W$ is a monoidal transformation with center $Z$, then the exceptional locus $H_{1} \subset W_{1}$ is a regular hypersurface, and if

$$
W_{2} \stackrel{e_{2}}{\longrightarrow} W_{1} \stackrel{e_{1}}{\longrightarrow} W
$$

is a composition of monoidal transformations with centers $Z \subset W$ and $Z_{1} \subset W_{1}$, then the exceptional locus of $e_{1} \circ e_{2}: W_{2} \longrightarrow W$ is the union of the strict transform of $H_{1}$ with the exceptional locus $H_{2}$ of $e_{2}: W_{2} \longrightarrow W_{1}$. Both Theorems 2.4 and 2.5 require the exceptional locus of $\pi: W^{\prime} \longrightarrow W$ to have normal crossings (see Definition 2.1 below), and to achieve this condition we will have to impose some constraints on the regular centers $Z \subset W$ and $Z_{1} \subset W_{1}$. With this purpose we introduce the notions of pairs and transformation of pairs which are suitable for the formulation of both Embedded Desingularization and Principalization of Ideals.

Definition 2.1. Let $W$ be a regular scheme and let $Y_{1}, \ldots, Y_{k} \subset W$ be a set of closed subschemes. We say that $Y_{1} \cup \ldots \cup Y_{k}$ have normal crossings at a point $\xi \in W$ if there exists a regular system of parameters $\left\{x_{1}, \ldots, x_{d}\right\} \subset$ $\mathcal{O}_{W, \xi}$, such that for each $i \in\{1, \ldots, k\}$, either $\mathcal{I}\left(Y_{i}\right)_{\xi}=\mathcal{O}_{W, \xi}$, or

$$
\mathcal{I}\left(Y_{i}\right)_{\xi}=\left\langle x_{i_{1}}, \ldots, x_{i_{s_{i}}}\right\rangle
$$

for some $x_{i_{1}}, \ldots, x_{i_{s_{i}}} \in\left\{x_{1}, \ldots, x_{d}\right\}$. We say that $Y_{1} \cup \ldots \cup Y_{k}$ have normal crossings in $W$ if they have normal crossings at any point of $W$. 
Definition 2.2. Let $W$ be a pure dimensional scheme, smooth over a field $\mathbf{k}$ of characteristic zero, and let $E=\left\{H_{1}, \ldots, H_{r}\right\}$ be a set of smooth hypersurfaces in $W$ with normal crossings. The couple $(W, E)$ is said to be a pair.

2.3. Transformation of pairs $([18,2.10]$, [35, 1.1]) A regular closed subscheme $Y \subset W$ is said to be permissible for the pair $(W, E)$ if $Y$ has normal crossings with $E$, (i.e. if $Y$ has normal crossings with $E=\left\{H_{1}, \ldots, H_{r}\right\}$ ). If $Y \subset W$ is permissible for a pair $(W, E)$, we define a transformation of pairs in the following way: Consider the blowing-up with center $Y$,

$$
W \stackrel{\Pi}{\longleftarrow} W_{1},
$$

and define $E_{1}=\left\{H_{1}^{\prime}, \ldots, H_{r}^{\prime}, H_{r+1}^{\prime}\right\}$, where $H_{i}^{\prime}$ denotes the strict transform of $H_{i}$, and $H_{r+1}^{\prime}$ denotes $\Pi^{-1}(Y)$, the exceptional hypersurface in $W_{1}$. Note that $W_{1}$ is smooth and that $E_{1}$ has normal crossings. We say that $(W, E) \longleftarrow\left(W_{1}, E_{1}\right)$ is a transformation of the pair $(W, E)$.

Now we state the two theorems that we prove:

Theorem 2.4 (Embedded resolution of singularities). Let $\left(W_{0}, E_{0}=\emptyset\right)$ be a pair and let $X_{0} \subset W_{0}$ be a closed equidimensional subscheme defined by $\mathcal{I}\left(X_{0}\right) \subset \mathcal{O}_{W_{0}}$. Assume that the open set $\operatorname{Reg}(X)$ of regular points of $X$ is dense in $X$. Then there exists a finite sequence of transformations of pairs

$$
\left(W_{0}, E_{0}=\emptyset\right) \longleftarrow \ldots \longleftarrow\left(W_{r}, E_{r}\right),
$$

inducing a proper birational morphism $\Pi_{r}: W_{r} \longrightarrow W_{0}$, such that if $E_{r}=$ $\left\{H_{1}, \ldots, H_{r}\right\}$ is the exceptional locus of $\Pi_{r}$ then:

(i) The strict transform of $X$ in $W_{r}, X_{r}$, is regular in $W_{r}$, and

$$
W_{r} \backslash \bigcup_{i=1}^{r} H_{i} \simeq W_{0}-\operatorname{Sing}(X) \text {. }
$$

In particular,

$$
\operatorname{Reg}(X) \cong \Pi_{r}^{-1}(\operatorname{Reg}(X)) \subset X_{r} \quad \text { via } \quad \Pi_{r} .
$$

(ii) The scheme $X_{r}$ has normal crossings with $E_{r}=\bigcup_{i=1}^{r} H_{i}$.

(iii) (Equivariance) If a group acting on $W$ is also acting on $X_{0} \subset W_{0}$, then the action can be lifted to one on $X_{r} \subset W_{r}$. 
Theorem 2.5 (Embedded Principalization of ideals). Let $\left(W_{0}, E_{0}\right)$ be a pair and let $I \subset \mathcal{O}_{W_{0}}$ be a non zero sheaf of ideals. Then there exists an embedded principalization of $I$, i.e. there is a finite sequence of transformations of pairs

$$
\left(W_{0}, E_{0}=\emptyset\right)=(W, E) \longleftarrow \ldots \longleftarrow\left(W_{r}, E_{r}\right),
$$

at smooth centers $Y_{i} \subset W_{i}$, such that:

(i) The morphism $W_{r} \rightarrow W$ defines an isomorphism over the open subset

$$
W \backslash V(I) .
$$

(ii) The ideal $\mathrm{IO}_{W_{r}}$ is invertible and supported on a divisor with normal crossings, i.e.,

$$
\mathcal{L}=I \mathcal{O}_{W_{r}}=\mathcal{I}\left(H_{1}\right)^{c_{1}} \cdot \ldots \cdot \mathcal{I}\left(H_{s}\right)^{c_{s}}
$$

where

$$
E^{\prime}=\left\{H_{1}, H_{2}, \ldots, H_{s}\right\}
$$

are regular hypersurfaces with normal crossings, $c_{i} \geq 1$ for $i=1, \ldots, s$, and

$$
E^{\prime}=E_{r}
$$

if $V(I)$ has no components of codimension 1.

(iii) (Equivariance) If a group $G$ acts on $W_{0}$ and on the ideal $I$, then it acts on each center $Y_{i}$ for $i=0, \ldots, r-1$, and the action can be lifted at each step of the resolution.

Remark 2.6. In particular, if $W=\mathbb{A}_{k}^{n}$, or any toric variety, $J$ is a monomial ideal and

$$
\left(W_{0}, E_{0}=\emptyset\right)=(W, E) \longleftarrow \ldots \longleftarrow\left(W_{r}, E_{r}\right),
$$

is an embedded principalization of $J$, obtained by blowing up at smooth centers $Y_{i} \subset W_{i}$ as indicated in Theorem 2.5, then all the ideals $\mathcal{I}\left(Y_{i}\right)$ are monomial ideals, because the sequence of transformations is equivariant, and the torus acts on $W$ and $J$, and hence on the centers $Y_{i}$, for $i=0,1, \ldots, r-1$.

The proofs of Theorems 2.4 and 2.5 will be given in 5.8 and 5.9 , where it will be shown that both theorems are a direct consequence of the existence of an algorithm of resolution of basic objects. Basic objects and algorithms of resolution of basic objects are discussed in the upcoming sections. 


\section{Part II. Basic objects}

\section{Basic objects}

In this section we recall the definitions of basic objects and resolution of basic objects (Definitions 3.1 and 3.8, see also [18]). Both Embedded Desingularization and Strong Principalization of Ideals can be obtained from a resolution of suitably defined basic objects.

Definition 3.1. A basic object is a triple that consists of a pair $(W, E)$, an ideal $J \subset \mathcal{O}_{W}$ such that $(J)_{\xi} \neq 0$ for any $\xi \in W$, and a positive integer $b$. It is denoted by $(W,(J, b), E)$. If the dimension of $W$ is $d$, then $(W,(J, b), E)$ is said to be a d-dimensional basic object.

Definition 3.2. The singular locus of a basic object is the closed subset of $W$,

$$
\operatorname{Sing}(J, b)=\left\{\xi \in W \mid \nu_{J}(\xi) \geq b\right\} \subset W,
$$

where $\nu_{J}(\xi)$ denotes the order of the ideal $J$ at the local regular ring $\mathcal{O}_{W, \xi}$. Sometimes we will use the notation

$$
\begin{gathered}
(W,(J, b), E) \\
\cup \\
\operatorname{Sing}(J, b)
\end{gathered}
$$

to refer to the basic object $(W,(J, b), E)$ together with the closed subset $\operatorname{Sing}(J, b)$, meaning that $\operatorname{Sing}(J, b) \subset W$.

Example 3.3. If $X \subset W$ is a hypersurface and $J$ is its defining ideal, then $\operatorname{Sing}(J, b)$ is the set of points of $X$ where the multiplicity is greater than or equal to $b$.

Example 3.4. If $J \subset \mathcal{O}_{W}$ is an arbitrary non-zero sheaf of ideals, then $\operatorname{Sing}(J, b)$ is the set of points of $W$ where the order of $J$ is greater than or equal to $b$.

Definition 3.5. A regular closed subscheme $Y \subset W$ is permissible for $(W,(J, b), E)$ if $Y$ is permissible for the pair $(W, E)$ and $Y \subset \operatorname{Sing}(J, b)$.

3.6. Permissible transformations of basic objects. Let $(W,(J, b), E=$ $\left.\left\{H_{1}, \ldots, H_{r}\right\}\right)$ be a basic object and let $Y \subset \operatorname{Sing}(J, b)$ be a permissible center. Consider $W \longleftarrow W_{1}$ the monoidal transformation with center $Y$. This induces a transformation of pairs,

$$
\left(W, E=\left\{H_{1}, \ldots, H_{r}\right\}\right) \longleftarrow\left(W_{1}, E_{1}=\left\{H_{1}, \ldots, H_{r}, H_{r+1}\right\}\right),
$$

where $H_{r+1} \subset W_{1}$ is the exceptional divisor (see Definition 2.2). 
Now, since $Y \subset \operatorname{Sing}(J, b)$,

$$
J \mathcal{O}_{W_{1}}=\mathcal{I}\left(H_{r+1}\right)^{b} J_{1},
$$

for some $J_{1} \subset \mathcal{O}_{W_{1}}$. Then we define

$$
(W,(J, b), E) \longleftarrow\left(W_{1},\left(J_{1}, b\right), E_{1}\right)
$$

as the permissible transformation of the basic object $(W,(J, b), E)$.

Remark 3.7. In general, given a sequence of transformations of basic objects

$$
\begin{aligned}
& \left(W_{0},\left(J_{0}, b\right), E_{0}\right) \longleftarrow\left(W_{1},\left(J_{1}, b\right), E_{1}\right) \longleftarrow \ldots \longleftarrow\left(W_{k},\left(J_{k}, b\right), E_{k}\right) \\
& \cup \cup \cup \\
& \operatorname{Sing}\left(J_{0}, b\right) \quad \operatorname{Sing}\left(J_{1}, b\right) \quad \ldots \quad \operatorname{Sing}\left(J_{k}, b\right)
\end{aligned}
$$

at centers $Y_{i} \subset \operatorname{Sing}\left(J_{i}, b\right), i=0,1, \ldots k-1$, we obtain expressions

$$
J_{0} \mathcal{O}_{W_{i}}=\mathcal{I}\left(H_{r+1}\right)^{c_{r+1}} \cdot \ldots \cdot \mathcal{I}\left(H_{r+i}\right)^{c_{r+i}} J_{i}
$$

Note here that $c_{r+1}=\cdots=c_{r+i}=b$ if none of the centers $Y_{i}$ are included in any of the exceptional divisors $H_{j}$.

Definition 3.8. A finite sequence transformation of basic objects as (3.7.1) is a resolution of $\left(W_{0},\left(J_{0}, b\right), E_{0}\right)$ if $\operatorname{Sing}\left(J_{k}, b\right)=\emptyset$.

\section{Remark 3.9. Note that:}

1. If sequence (3.7.1) is a resolution of the basic object $\left(W_{0},\left(J_{0}, b\right), E_{0}\right)$, then $W_{k} \longrightarrow W_{0}$ defines an isomorphism over $W_{0} \backslash V\left(J_{0}\right)$, and $J_{0} \mathcal{O}_{W_{k}}=$ $\mathcal{M}_{k} J_{k}$, where $\mathcal{M}_{k}$ is an invertible sheaf of ideals, and $J_{k}$ has no points of order $\geq b$ in $W_{k}$.

2. The ideal $J_{k}$ is not the strict transform of $J_{0}$, an ideal which is far more complicated to define (see Section 7 for a discussion on this matter). However it is so in some particular cases. In fact, if $X_{0} \subset W_{0}$ is a closed smooth subscheme, and $J_{0}=I(X)$, then $\operatorname{Sing}\left(J_{0}, 1\right)=X$, and given any sequence of transformations of basic objects

$$
\left(W_{0},\left(J_{0}, 1\right), E_{0}\right) \longleftarrow\left(W_{1},\left(J_{1}, 1\right), E_{1}\right) \longleftarrow \ldots \longleftarrow\left(W_{k},\left(J_{k}, 1\right), E_{k}\right)
$$

the ideal $J_{k}$ is the ideal of a smooth subscheme $X_{k} \subset W_{k}$, which is the strict transform of $X \subset W_{0}$. 
Theorem 3.10. [35, Theorem 7.3] [18, Theorem 7.13] Given a basic object $(W,(J, b), E)$ where $W$ is smooth over a field of characteristic zero, there is a resolution, i.e. there is a finite sequence of monoidal transformations at permissible centers $Y_{i} \subset W_{i}$,

$(W,(J, b), E)=\left(W_{0},\left(J_{0}, b\right), E_{0}\right) \leftarrow\left(W_{1},\left(J_{1}, b\right), E_{1}\right) \leftarrow \ldots \leftarrow\left(W_{k},\left(J_{k}, b\right), E_{k}\right)$, such that $\operatorname{Sing}\left(J_{k}, b\right)=\emptyset$.

Remark 3.11. Note that:

(i) Theorem 3.10 is existential. It claims that given $(W,(J, b), E)$ there exists a resolution. However we shall give a constructive proof (the same as in [35, Theorem 7.3], [18, Theorem 7.13]), so that given $(W,(J, b), E)$ we will define one particular resolution.

(ii) We will show in 5.8 and 5.9 that a constructive proof of Theorem 3.10 will lead us to simple constructive proofs of Theorems 2.4 and 2.5.

A central point in our constructive proof of Theorem 3.10, and hence of Theorems 2.4 and 2.5, relies on the fact that all the invariants involved in the algorithmic resolution of a basic object $(W,(J, b), E)$, will be defined in terms of the closed sets of the form $F=\operatorname{Sing}(J, b)$. The definition of these invariants will be addressed in Sections 6 and 17. As it turns out, the invariants that we are aiming to use do not behave well if only transformations as the ones of Definition 3.6 were allowed (see Section 4 for equivariance, and Remark 6.9 where this fact is illustrated). This forces us to enlarge the class of transformations of basic objects by including the projections.

3.12. Projections. Let $(W,(J, b), E)$ be a basic object. We will define a new notion of transformation of pairs and of basic objects as follows: Set

$$
W_{1}=W \times \mathbb{A}_{k}^{1}
$$

and consider the natural projection $p: W_{1} \longrightarrow W$. Then define

$$
J_{1}=J \mathcal{O}_{W_{1}} \text { and } E_{1}=p^{-1}(E) .
$$

This gives us a transformation of pairs

$$
(W, E) \longleftarrow\left(W_{1}, E_{1}\right),
$$

and a transformation of basic objects,

$$
(W,(J, b), E) \longleftarrow\left(W_{1},\left(J_{1}, b\right), E_{1}\right) .
$$

In this case the closed subset in $W_{1}$ is $F_{1}=\operatorname{Sing}\left(J_{1}, b\right)=p^{-1}(\operatorname{Sing}(J, b))$. 
In what follows, unless otherwise specified, whenever we mention a transformation or a sequence of transformations of basic objects, we will refer to both the permissible transformations introduced in Definition 3.6 and the projections that we have just defined. In both cases we will use the same notation,

$$
\begin{aligned}
& \left(W_{0},\left(J_{0}, b\right), E_{0}\right) \longleftarrow\left(W_{1},\left(J_{1}, b\right), E_{1}\right) \longleftarrow \ldots \longleftarrow\left(W_{k},\left(J_{k}, b\right), E_{k}\right) \\
& \cup \quad \cup \quad \cup \\
& \operatorname{Sing}\left(J_{0}, b\right) \quad \operatorname{Sing}\left(J_{1}, b\right) \quad \ldots \quad \operatorname{Sing}\left(J_{k}, b\right),
\end{aligned}
$$

and we will assume that both kinds of transformations can be used in the same sequence.

\section{Equivariance}

An important outcome of constructive desingularization is the lifting of any group action on a subscheme $X \subset W$, all the way up to the desingularization. Since our desingularization theorem will follow from a suitably defined resolution of basic objects, in this section we present and discuss the notion of equivariance in the context of basic objects. We should point out that we will work with isomorphisms $\Theta: W \rightarrow W^{\prime}$ which may be not necessarily defined over the base field $\mathbf{k}$ over which the smooth schemes $W$ and $W^{\prime}$ are defined.

Definition 4.1. An isomorphism of pairs

$$
\Theta:\left(W, E=\left\{H_{1}, \ldots, H_{k}\right\}\right) \longrightarrow\left(W^{\prime}, E^{\prime}=\left\{H_{1}^{\prime}, \ldots, H_{k}^{\prime}\right\}\right)
$$

is an isomorphism $\Theta: W \longrightarrow W^{\prime}$ such that $\Theta\left(H_{i}\right)=H_{i}^{\prime}$ for all $i=1, \ldots, k$.

Lemma 4.2. Let $\Theta:(W, E) \longrightarrow\left(W^{\prime}, E^{\prime}\right)$ be an isomorphism of pairs, let $Y$ be a permissible center for $(W, E)$ and set $Y^{\prime}=\Theta(Y)$. Consider the transformations of pairs with centers $Y$,

$$
(W, E) \longleftarrow\left(W_{1}, E_{1}\right),
$$

and $Y^{\prime}$,

$$
\left(W^{\prime}, E^{\prime}\right) \longleftarrow\left(W_{1}^{\prime}, E_{1}^{\prime}\right) .
$$

Then there is a natural lifting of the isomorphism $\Theta:(W, E) \longrightarrow\left(W^{\prime}, E^{\prime}\right)$ to an isomorphism

$$
\Theta_{1}:\left(W_{1}, E_{1}\right) \longrightarrow\left(W_{1}^{\prime}, E_{1}^{\prime}\right)
$$

such that the diagram

$$
\begin{array}{ccc}
(W, E) & \longleftarrow & \left(W_{1}, E_{1}\right) \\
\Theta \downarrow & & \downarrow \Theta_{1} \\
\left(W^{\prime}, E^{\prime}\right) & \longleftarrow & \left(W_{1}^{\prime}, E_{1}^{\prime}\right)
\end{array}
$$

commutes. 
Proof: The map $\Theta:(W, E) \longrightarrow\left(W^{\prime}, E^{\prime}\right)$ induces an isomorphism of the corresponding structural sheaves $\mathcal{O}_{W}$ and $\mathcal{O}_{W^{\prime}}$, and extends naturally to an isomorphism

$$
\hat{\Theta}: W \times \mathbb{A}_{\mathbf{k}^{1}} \longrightarrow W^{\prime} \times \mathbb{A}_{\mathbf{k}^{1}},
$$

i.e. an isomorphism between $\mathcal{O}_{W}[Z]$ and $\mathcal{O}_{W^{\prime}}\left[Z^{\prime}\right]$, where $Z$ and $Z^{\prime}$ are indeterminates and $\Theta(Z)=Z^{\prime}$.

Since $\Theta(Y)=Y^{\prime}$, the sheaf of ideals $\mathcal{I}(Y) \subset \mathcal{O}_{W}$ is mapped to $\mathcal{I}\left(Y^{\prime}\right) \subset \mathcal{O}_{W^{\prime}}$. As a consequence, $\Theta$ induces an isomorphism $\mathcal{O}_{W}[I(Y) Z] \simeq \mathcal{O}_{W^{\prime}}\left[I\left(Y^{\prime}\right) Z^{\prime}\right]$ which preserves the grading. Therefore we get an isomorphism

$$
W_{1}=\operatorname{Proj}\left(\mathcal{O}_{W}[I(Y) Z]\right) \simeq W_{1}^{\prime}=\operatorname{Proj}\left(\mathcal{O}_{W^{\prime}}\left[I\left(Y^{\prime}\right) Z^{\prime}\right]\right) .
$$

We finally check that this isomorphism maps the exceptional hypersurface of one to that of the other, defining $\Theta_{1}:\left(W_{1}, E_{1}\right) \longrightarrow\left(W_{1}^{\prime}, E_{1}^{\prime}\right)$.

Remark 4.3. Let $\Theta:(W, E) \rightarrow\left(W^{\prime}, E^{\prime}\right)$ be an isomorphism of pairs. Set $W_{1}=W \times \mathbb{A}_{k}^{1}, W_{1}^{\prime}=W^{\prime} \times \mathbb{A}_{k}^{1}$, both as in 3.12; and set $\Theta_{1}=\Theta \times \operatorname{Id}_{\mathbb{A}_{k}^{1}}$. Note that $\Theta_{1}:\left(W_{1}, E_{1}\right) \rightarrow\left(W_{1}^{\prime}, E_{1}^{\prime}\right)$ is an isomorphism of pairs.

Remark 4.4. Fix an isomorphism $\Theta:(W, E) \longrightarrow\left(W^{\prime}, E^{\prime}\right)$ and a sequence

$$
(W, E) \longleftarrow\left(W_{1}, E_{1}\right) \longleftarrow \ldots \longleftarrow\left(W_{k}, E_{k}\right)
$$

where each transformations of pairs is either as in 2.3 or as in 3.12. Then Lemma 4.2 and Remark 4.3 assert that $\Theta: W \longrightarrow W^{\prime}$ defines a unique sequence of transformation of pairs,

$$
\left(W^{\prime}, E^{\prime}\right) \longleftarrow\left(W_{1}^{\prime}, E_{1}^{\prime}\right) \longleftarrow \ldots \longleftarrow\left(W_{k}^{\prime}, E_{k}^{\prime}\right)
$$

together with isomorphisms $\Theta_{i}:\left(W_{i}, E_{i}\right) \longrightarrow\left(W_{i}^{\prime}, E_{i}^{\prime}\right)$, for $i=1, \ldots, k$.

Remark 4.5. Let $\mathcal{B}=(W,(J, b), E)$ be a basic object. If $U \subset W$ is an open set, then we set the restriction of the basic object to be $\left.\mathcal{B}\right|_{U}=$ $\left(U,\left(\left.J\right|_{U}, b\right), E_{U}\right)$, where $\left.J\right|_{U}$ is the restriction of the sheaf of ideal to $U$ and $E_{U}=\{H \cap U \mid H \in E\}$.

Definition 4.6. Let $(W,(J, b), E)$ and $\left(W^{\prime},\left(J^{\prime}, b^{\prime}\right), E^{\prime}\right)$ be two basic objects and let

$$
\Theta:(W, E) \rightarrow\left(W^{\prime}, E^{\prime}\right)
$$

be an isomorphism of pairs. We will say that $\Theta$ induces an isomorphism of basic objects,

$$
\Theta:(W,(J, b), E) \rightarrow\left(W^{\prime},\left(J^{\prime}, b^{\prime}\right), E^{\prime}\right),
$$

if the following conditions hold: 
(i) The isomorphism $\Theta: W \rightarrow W^{\prime}$ induces an isomorphism of the closed subsets defined by the basic objects

$$
\Theta: F=\operatorname{Sing}(J, b) \cong F^{\prime}=\operatorname{Sing}\left(J^{\prime}, b^{\prime}\right) .
$$

(ii) Given a sequence of transformations of basic objects as in 3.6 or 3.12, $(4.6 .1)$

$$
(W,(J, b), E) \longleftarrow\left(W_{1},\left(J_{1}, b\right), E_{1}\right) \longleftarrow \ldots \longleftarrow\left(W_{k},\left(J_{k}, b\right), E_{k}\right),
$$

together with the corresponding sequence of transformation of pairs

$$
(W, E) \longleftarrow\left(W_{1}, E_{1}\right) \longleftarrow \ldots \longleftarrow\left(W_{k}, E_{k}\right)
$$

then:

(a) The corresponding induced sequence, as in Remark 4.4,

$$
\left(W^{\prime}, E^{\prime}\right) \longleftarrow\left(W_{1}^{\prime}, E_{1}^{\prime}\right) \longleftarrow \ldots \longleftarrow\left(W_{k}^{\prime}, E_{k}^{\prime}\right)
$$

defines a sequence of transformation of basic objects,

$$
\left(W^{\prime},\left(J^{\prime}, b^{\prime}\right), E^{\prime}\right) \leftarrow\left(W_{1}^{\prime},\left(J_{1}^{\prime}, b^{\prime}\right), E_{1}^{\prime}\right) \leftarrow \cdots \leftarrow\left(W_{k}^{\prime},\left(J_{k}^{\prime}, b^{\prime}\right), E_{k}^{\prime}\right)
$$

(b) The isomorphisms $\Theta_{i}:\left(W_{i}, E_{i}\right) \rightarrow\left(W_{i}^{\prime}, E_{i}^{\prime}\right)$ defined in remark 4.4, induce an isomorphism of the closed subsets defined by the basic objects,

$$
\Theta_{i}: \operatorname{Sing}\left(J_{i}, b\right) \cong \operatorname{Sing}\left(J_{i}^{\prime}, b^{\prime}\right) \quad \text { for all } i=1, \ldots, k .
$$

(iii) For any open set $U$, set $U^{\prime}=\Theta(U)$ and consider the restrictions $\left(U,\left(\left.J\right|_{U}, b\right), E_{U}\right)$ and $\left(U^{\prime},\left(\left.J^{\prime}\right|_{U^{\prime}}, b^{\prime}\right), E_{U}^{\prime}\right)$. We require that properties (i) and (ii) hold for these restrictions.

Example 4.7. Let $\Theta:(W, E) \rightarrow\left(W^{\prime}, E^{\prime}\right)$ be an isomorphism of pairs, fix a basic object $(W,(J, b), E)$ and let $J^{\prime}$ be the image of $J$ in $\mathcal{O}_{W}^{\prime}$ under $\Theta$, i.e. $J^{\prime}=\Theta(J) \subset \mathcal{O}_{W^{\prime}}$. Then we claim that

$$
\Theta:(W,(J, b), E) \rightarrow\left(W^{\prime},\left(J^{\prime}, b\right), E^{\prime}\right)
$$

is an isomorphism of basic objects: Clearly $\Theta: W \rightarrow W^{\prime}$ maps $\operatorname{Sing}(J, b)$ isomorphically into $\operatorname{Sing}\left(J^{\prime}, b\right)$. To check that condition (ii) of Definition 4.6 holds, note that if

$$
(W,(J, b), E) \longleftarrow\left(W_{1},\left(J_{1}, b\right), E_{1}\right)
$$

is a transformation of basic objects as the ones defined in 3.12, then the corresponding transformation of basic objects over $\left(W^{\prime},\left(J^{\prime}, b\right), E^{\prime}\right)$,

$$
\left(W^{\prime},\left(J^{\prime}, b\right), E^{\prime}\right) \longleftarrow\left(W_{1}^{\prime},\left(J_{1}^{\prime}, b\right), E_{1}^{\prime}\right)
$$


is such that the there is a natural way to define an isomorphism

$$
\Theta_{1}: W_{1} \rightarrow W_{1}^{\prime}
$$

which maps $\operatorname{Sing}\left(J_{1}, b\right)$ isomorphically into $\operatorname{Sing}\left(J_{1}^{\prime}, b\right)$. In fact, $\Theta_{1}$ maps $J_{1}=J \mathcal{O}_{W_{1}}$ into $J_{1}^{\prime}=J^{\prime} \mathcal{O}_{W_{1}^{\prime}}$.

Now if

$$
(W,(J, b), E) \longleftarrow\left(W_{1},\left(J_{1}, b\right), E_{1}\right)
$$

is a transformation of basic objects with center $Y$ as in Definition 3.6, and if

$$
\left(W^{\prime},\left(J^{\prime}, b\right), E^{\prime}\right) \longleftarrow\left(W_{1}^{\prime},\left(J_{1}^{\prime}, b\right), E_{1}^{\prime}\right)
$$

is a transformation of basic objects with center $Y^{\prime}=\Theta(Y)$, then by Lemma 4.2 we obtain an isomorphism of pairs

$$
\Theta_{1}:\left(W_{1}, E_{1}\right) \rightarrow\left(W_{1}^{\prime}, E_{1}^{\prime}\right)
$$

Since $\Theta_{1}: W_{1} \cong W_{1}^{\prime}$ is compatible with $\Theta: W \cong W^{\prime}$ it follows that

$$
\Theta_{1}\left(J \mathcal{O}_{W_{1}}\right)=J^{\prime} \mathcal{O}_{W_{1}^{\prime}}
$$

In particular, by (3.6.1), $\Theta_{1}\left(J_{1}\right)=J_{1}^{\prime}$, and hence $\Theta_{1}\left(\operatorname{Sing}\left(J_{1}, b\right)\right)=\operatorname{Sing}\left(J_{1}^{\prime}, b\right)$.

Example 4.8. The identity map induces an isomorphism of the basic objects,

$$
\text { Id }:(W,(J, b), E) \rightarrow\left(W,\left(J^{2}, 2 b\right), E\right),
$$

(see also Example 12.6).

Remark 4.9. Note that if two basic objects are isomorphic, then there might be may different isomorphisms between them. We are interested in resolutions which are compatible with any of these isomorphisms. This is the philosophy behind the next definition.

Definition 4.10. Let $(W,(J, b), E)$ be a basic object. We will say that a sequence of transformations of basic objects,

$$
(W,(J, b), E) \longleftarrow\left(W_{1},\left(J_{1}, b\right), E_{1}\right) \longleftarrow \ldots \longleftarrow\left(W_{k},\left(J_{k}, b\right), E_{1}\right)
$$

is equivariant, if for any isomorphic basic object $\left(W^{\prime},\left(J^{\prime}, b\right), E^{\prime}\right)$, the induced sequence of transformations defined as in (4.6.2),

$$
\left(W^{\prime},\left(J^{\prime}, b^{\prime}\right), E^{\prime}\right) \longleftarrow\left(W_{1}^{\prime},\left(J_{1}^{\prime}, b^{\prime}\right), E_{1}^{\prime}\right) \longleftarrow \ldots \longleftarrow\left(W_{k}^{\prime},\left(J_{k}^{\prime}, b^{\prime}\right), E_{1}^{\prime}\right),
$$

is independent of the isomorphism $\Theta:(W,(J, b), E) \rightarrow\left(W^{\prime},\left(J^{\prime}, b^{\prime}\right), E^{\prime}\right)$ that we take. 
4.11. What does equivariance mean? In Section 5 we will introduce the notion of algorithm of resolution of basic objects. The algorithm will provide, for each basic object, a resolution which is equivariant, i.e., let

$$
\begin{aligned}
& \left(W_{0},\left(J_{0}, b\right), E_{0}\right) \longleftarrow\left(W_{1},\left(J_{1}, b\right), E_{1}\right) \longleftarrow \ldots \longleftarrow\left(W_{k},\left(J_{k}, b\right), E_{k}\right) \\
& \cup \quad \cup \quad \cup \\
& \operatorname{Sing}\left(J_{0}, b\right) \quad \operatorname{Sing}\left(J_{1}, b\right) \quad \ldots \quad \operatorname{Sing}\left(J_{k}, b\right)=\emptyset
\end{aligned}
$$

be the resolution of $\mathcal{B}=\left(W_{0},\left(J_{0}, b\right), E_{0}\right)$ provided by the algorithm, and let

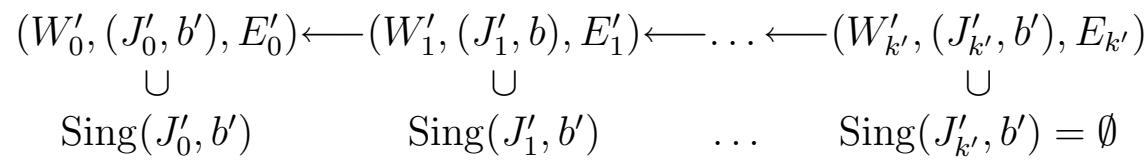

be that of $\mathcal{B}^{\prime}=\left(W_{0}^{\prime},\left(J_{0}^{\prime}, b^{\prime}\right), E^{\prime}\right)$. We require that if $\mathcal{B}$ and $\mathcal{B}^{\prime}$ are isomorphic, i.e. if

$$
\Theta:\left(W_{0},\left(J_{0}, b\right), E_{0}\right) \rightarrow\left(W_{0}^{\prime},\left(J_{0}^{\prime}, b\right), E_{0}^{\prime}\right)
$$

is an isomorphism, then sequence (4.10.1) induced by (4.11.1) is precisely the sequence (4.11.2), and hence the isomorphism can be lifted along the resolutions (4.11.1) and (4.11.2).

Since the resolutions are equivariant, $\Theta$ can be lifted, and furthermore, any other isomorphism $\Gamma: \mathcal{B} \rightarrow \mathcal{B}^{\prime}$ can also be lifted. An algorithm with this property is said to be an equivariant algorithm. This property, and others, will arise, in a very simple manner, by defining the resolution in terms of upper semi-continuous functions with suitable properties.

\section{The algorithmic proof of Theorems 2.5 and 2.4}

In this section we explain what we mean by a constructive or algorithmic proof of Theorem 3.10. This constructive proof will be done in terms of an algorithm of resolution of basic objects (see Definition 5.5). In order to define such an algorithm we first introduce a totally ordered set, and then we attach to each basic object an upper semi-continuous function with values on this ordered set. These upper semi-continuous functions will define a resolution for each basic object $(W,(J, b), E)$, whenever $W$ is defined over a field of characteristic zero. We will refer to this resolution as the resolution defined by the algorithm. At the end of the section we will indicate why an algorithm of resolution of basic objects as the one described in Definition 5.5 already provides a proof of Theorems 2.4 and 2.5 (see 5.8 and 5.9). 
Definition 5.1. Let $X$ be a topological space, let $(T, \geq)$ be a totally ordered set, and let $g: X \longrightarrow T$ be an upper semi-continuous function. Assume that $g$ takes only finitely many values. Then the largest value achieved by $g$ will be denoted by

$\max g$.

Clearly the set

$$
\underline{\operatorname{Max}} g=\{x \in X: g(x)=\max g\}
$$

is a closed subset of $X$.

Definition 5.2. Let $T$ be a totally ordered set. Assume that for any basic object $\mathcal{B}=(W,(J, b), E)$ there is an upper semi-continuous function

$$
f_{\mathcal{B}}: \operatorname{Sing}(J, b) \rightarrow T
$$

associated to it. This is what we will call a family of functions with values on $T$. Given a sequence of transformations of basic objects,

$$
\left(W_{k},\left(J_{k}, b\right), E_{k}\right) \longleftarrow \ldots \longleftarrow\left(W_{1},\left(J_{1}, b\right), E_{1}\right) \longleftarrow\left(W_{0},\left(J_{0}, b\right), E_{0}\right),
$$

we will denote by $f_{i}$ the corresponding function

$$
f_{\mathcal{B}_{i}}: \operatorname{Sing}\left(J_{i}, b\right) \rightarrow T
$$

associated to $\mathcal{B}_{i}=\left(W_{i},\left(J_{i}, b\right), E_{i}\right)$.

Definition 5.3. A family of functions is said to be equivariant if for any isomorphism of basic objects

$$
\Theta: \mathcal{B}=(W,(J, b), E) \rightarrow \mathcal{B}^{\prime}=\left(W^{\prime},\left(J^{\prime}, b\right), E^{\prime}\right),
$$

we have that

$$
f_{\mathcal{B}^{\prime}} \circ \Theta=f_{\mathcal{B}} .
$$

Remark 5.4. A family of functions with values on $T$ attaches to each basic object a unique function. An isomorphism $\Theta$ as in Definition 5.3 defines an isomorphism $W \cong W^{\prime}$ mapping the domain of $f_{\mathcal{B}}$ into the domain of $f_{\mathcal{B}^{\prime}}$, and in particular, mapping

$$
\operatorname{Sing}(J, b) \subset W
$$

isomorphically into

$$
\operatorname{Sing}\left(J^{\prime}, b\right) \subset W^{\prime}
$$

so formula (5.3.1) makes sense. 
Definition 5.5. An algorithm of resolution of d-dimensional basic objects consists of:

(A) A totally ordered set $\left(I_{d}, \leq\right)$.

(B) A family of functions with values on $\left(I_{d}, \leq\right)$,

$$
f_{\mathcal{B}}: \operatorname{Sing}(J, b) \rightarrow I_{d}
$$

which satisfies the following properties:

(a) The closed subset

$\underline{\operatorname{Max}} f_{\mathcal{B}}$

is a smooth permissible center for $(W,(J, b), E)$, and it therefore defines a transformation of basic objects

$$
(W,(J, b), E) \longleftarrow\left(W_{1},\left(J_{1}, b\right), E_{1}\right) .
$$

Hence, given a basic object $\left(W_{0},\left(J_{0}, b\right), E_{0}\right)$, by this property we always get a finite sequence of transformations of basic objects

$$
\begin{aligned}
& \left(W_{0},\left(J_{0}, b\right), E_{0}\right) \longleftarrow\left(W_{1},\left(J_{1}, b\right), E_{1}\right) \longleftarrow \ldots \longleftarrow\left(W_{k},\left(J_{k}, b\right), E_{k}\right) \\
& \cup \cup \cup \\
& \operatorname{Sing}\left(J_{0}, b\right) \quad \operatorname{Sing}\left(J_{1}, b\right) \quad \ldots \quad \operatorname{Sing}\left(J_{k}, b\right),
\end{aligned}
$$

with centers $\underline{\operatorname{Max}} f_{i} \subset \operatorname{Sing}\left(J_{i}, b\right)$.

(b) For any sequence as (5.5.1) we have that

$$
\max f_{0}>\max f_{1}>\cdots>\max f_{k} .
$$

(c) For any sequence as (5.5.1) there is an index $N$, depending on $\left(W_{0},\left(J_{0}, b\right), E_{0}\right)$, such that

$$
\operatorname{Sing}\left(J_{N}, b\right)=\emptyset \text {. }
$$

(d) If $X_{0}$ is a regular pure dimensional subscheme of dimension $r$ then there is a value $a(r) \in I_{d}$ so that if $J_{0}=\mathcal{I}\left(X_{0}\right), b=1$ and $E_{0}=\emptyset$, then the function $f_{0}$ is constant and equal to $a(r)$.

(e) If $\xi \in \operatorname{Sing}\left(J_{i}, b\right)$ and $\xi \notin \underline{\operatorname{Max}} f_{i}$ for $i=0, \ldots, k-1$, then $f_{i}(\xi)=$ $f_{i+1}\left(\xi^{\prime}\right)$ via the natural identification of the point $\xi$ with a point $\xi^{\prime}$ of $\operatorname{Sing}\left(J_{i+1}, b\right)$.

(f) The family of functions $f_{\mathcal{B}}$ is equivariant: If

$$
\Theta:(W,(J, b), E) \longrightarrow\left(W^{\prime},\left(J^{\prime}, b^{\prime}\right) E^{\prime}\right)
$$

is an isomorphism of basic objects, then the functions given by the algorithm

$$
f: \operatorname{Sing}(J, b) \rightarrow T \quad \text { and } \quad f^{\prime}: \operatorname{Sing}\left(J^{\prime}, b\right) \rightarrow T
$$

are such that $f^{\prime} \circ \Theta=f$. 


\subsection{Some comments on the properties of the algorithm.}

(1) Property (c) says that for $i=0,1, \ldots, k$, the functions

$$
f_{i}: \operatorname{Sing}\left(J_{i}, b\right) \rightarrow I_{d}
$$

define a resolution of the basic object $\left(W_{0},\left(J_{0}, b\right), E_{0}\right)$. We will refer to it as the resolution defined by the algorithm.

(2) Compatibility with open restrictions: Let $U \subset W$ be an open subset and let

$$
(W,(J, b), E)_{U}=\left(U,\left(\left.J\right|_{U}, b\right), E_{U}\right)
$$

be the restriction to $U$ of $(W,(J, b), E)$. Property (e) asserts that the restriction to $U$ of the resolution of $(W,(J, b), E)$ defined by the algorithm, coincides with the resolution of the basic object $(W,(J, b), E)_{U}$ defined by the algorithm.

(3) Let

$$
\Theta_{0}:\left(W_{0},\left(J_{0}, b\right), E_{0}\right) \rightarrow\left(W_{0}^{\prime},\left(J_{0}^{\prime}, b^{\prime}\right), E_{0}^{\prime}\right)
$$

be an isomorphism of basic objects. Now consider on the one hand the resolution of $\left(W_{0},\left(J_{0}, b\right), E_{0}\right)$,

$$
\begin{aligned}
& \left(W_{0},\left(J_{0}, b\right), E_{0}\right) \longleftarrow\left(W_{1},\left(J_{1}, b\right), E_{1}\right) \longleftarrow \ldots \longleftarrow\left(W_{k},\left(J_{k}, b\right), E_{k}\right) \\
& \cup \cup \cup \\
& \operatorname{Sing}\left(J_{0}, b\right) \quad \operatorname{Sing}\left(J_{1}, b\right) \quad \ldots \quad \operatorname{Sing}\left(J_{k}, b\right)=\emptyset,
\end{aligned}
$$

defined by the upper semi-continuous functions

$$
f_{i}: \operatorname{Sing}\left(J_{i}, b\right) \rightarrow I_{d},
$$

and on the other hand the resolution of $\left(W_{0}^{\prime},\left(J_{0}^{\prime}, b^{\prime}\right), E_{0}^{\prime}\right)$,

$$
\begin{aligned}
& \left(W_{0}^{\prime},\left(J_{0}^{\prime}, b^{\prime}\right), E_{0}^{\prime}\right) \longleftarrow\left(W_{1}^{\prime},\left(J_{1}^{\prime}, b^{\prime}\right), E_{1}^{\prime}\right) \longleftarrow \ldots \longleftarrow\left(W_{k}^{\prime},\left(J_{k}^{\prime}, b\right), E_{k}^{\prime}\right) \\
& \operatorname{Sing}\left(J_{0}^{\prime}, b^{\prime}\right) \quad \operatorname{Sing}\left(J_{1}^{\prime}, b^{\prime}\right) \quad \ldots \quad \operatorname{Sing}\left(J_{k}^{\prime}, b^{\prime}\right)=\emptyset,
\end{aligned}
$$

defined by the upper semi-continuous functions

$$
f_{i}^{\prime}: \operatorname{Sing}\left(J_{i}, b\right) \rightarrow I_{d}
$$

Then condition (f) asserts that

$$
f_{i}(x)=f_{i}^{\prime}\left(\Theta_{i}(x)\right)
$$


for all $x \in \operatorname{Sing}\left(J_{i}, b\right)$ and in particular

$$
\Theta_{i}: \underline{\operatorname{Max}} f_{i} \rightarrow \underline{\operatorname{Max}} f_{i}
$$

is an isomorphism. Note that this guarantees that

$$
\Theta_{i}:\left(W_{i},\left(J_{i}, b\right), E_{i}\right) \rightarrow\left(W_{i}^{\prime},\left(J_{i}^{\prime}, b^{\prime}\right), E_{i}^{\prime}\right)
$$

lifts to an isomorphism

$$
\Theta_{i+1}:\left(W_{i+1},\left(J_{i+1}, b\right), E_{i+1}\right) \longrightarrow\left(W_{i+1}^{\prime},\left(J_{i+1}^{\prime}, b^{\prime}\right) E_{i+1}^{\prime}\right) .
$$

Therefore sequences (5.6.2) and (5.6.3) are linked as (4.6.2) is to (4.6.1). Furthermore, the same holds for any isomorphism $\Gamma: \mathcal{B} \rightarrow \mathcal{B}^{\prime}$.

Note that the previous isomorphism $\Theta_{i}$ in (5.6.4) links functions on two basic objects, both with the same index $i$.

Remark 5.7. The construction of the $i$-th function

$$
f_{i}: \operatorname{Sing}\left(J_{i}, b\right) \rightarrow I_{d}
$$

defined by the algorithm depends on the previous functions $f_{j}: \operatorname{Sing}\left(J_{j}, b\right) \rightarrow$ $I_{d}$, for $j \in\{0,1, \ldots, i-1\}$. This is not explicitly said in the definition of algorithm, but this is only a technical fact. Note that in any case the previous isomorphism $\Theta_{i}$ in (5.6.4) links functions on two basic objects, both with the same index $i$.

5.8. Proof of Theorem 2.4. With the same notation as in Theorem 2.4, consider the basic object

$$
\left(W_{0},\left(J_{0}, 1\right), E_{0}\right),
$$

where $W_{0}=W, J_{0}=\mathcal{I}(X)$ and $E_{0}=\emptyset$. Clearly $X=\operatorname{Sing}\left(J_{0}, 1\right)$.

By Definition $5.5(\mathrm{~d})$ and $5.6(2)$, the function

$$
f_{0}: \operatorname{Sing}\left(J_{0}, 1\right) \rightarrow\left(I_{d}, \leq\right)
$$

is constant on the restriction of $\left(W_{0},\left(J_{0}, 1\right), E_{0}\right)$ to $U=W \backslash \operatorname{Sing}(X)$. Let $a(d)$ denote this constant value along the points in $U \cap X$.

By Definition $5.5(\mathrm{c})$, we know that the algorithm provides a resolution of the basic object $\left(W_{0},\left(J_{0}, 1\right), E_{0}\right)$ by means of a finite sequence of blow-ups

$$
\left(W_{0},\left(J_{0}, 1\right), E_{0}\right) \longleftarrow\left(W_{1},\left(J_{1}, 1\right), E_{1}\right) \longleftarrow \ldots \longleftarrow\left(W_{N},\left(J_{N}, 1\right), E_{N}\right),
$$

at permissible centers $Y_{i} \subset \operatorname{Sing}\left(J_{i}, b\right)$ for $i=0,1, \ldots, N-1$. Therefore, there must be an index $k \in\{0,1, \ldots, N\}$ such that $\max f_{k}=a(d)$, and by Definition 5.5 (b), there is a unique index $k$ with this condition. 
Now $U$ can be identified with an open set, say $U$ again, of $W_{k}$ (note that the centers of the transformations in sequence (5.8.1) are defined by $\operatorname{Max} f_{i}$ and $\max f_{i}>a(d)$ for $i<k$ ). If $X_{k}$ denotes the strict transform of $X$ in $W_{k}$,

$$
X_{k} \cap U=X \cap U=\underline{\operatorname{Max}} f_{k} \cap U .
$$

Since $X \cap U=\operatorname{Reg}(X)$ is dense in $X$, it follows that $X_{k}$ is the union of some of the components of $\underline{\operatorname{Max}} f_{k}$, and hence it is regular and has normal crossings with the exceptional components by Definition 5.5 (a). This proves (i) and (ii) of Theorem 2.4.

Now it only remains to show that the resolution of singularities of $X$ that we have achieved is equivariant. An argument similar to the one in the proof of Lemma 4.2 shows that if a group $G$ acts on $W_{0}$, and $\Theta\left(Y_{0}\right)=Y_{0}$ for all $\Theta \in G$, then the group $G$ acts on the ideal $\mathcal{I}(Y) \subset \mathcal{O}_{W}$, and hence on the blow-up $W_{1}$. So in order to lift the action of $G$ to $W_{k}$, it suffices to lift the action of all the elements of the group $\Theta \in G$, step by step, to an isomorphism on $W_{k}$. By assumption $\Theta\left(X_{0}\right)=X_{0}$ so we can argue as in Example 4.7 to show that each element $\Theta \in G$ defines an isomorphism of the basic object $\left(W_{0},\left(J_{0}, 1\right), E_{0}\right)$ into itself. Hence part (iii) of Theorem 2.4 follows from $5.6(3)$.

5.9. Proof of Theorem 2.5. With the notation of Theorem 2.5, it is enough to consider the resolution of the basic object $(W,(I, 1), E)$ provided by Theorem 3.10 (see also Remark 3.9 (1)).

To show that the embedded principalization is equivariant we argue as in 5.8, where now each element $\Theta \in G$ defines an isomorphism of the basic object $\left(W_{0},\left(J_{0}, 1\right), E_{0}\right)=(W,(I, 1), E)$ into itself.

\section{The two main families of equivariant functions}

In Section 5 we have introduced the notion of resolution of basic objects, and we have shown how such a resolution can be applied to give a constructive proof of Theorems 2.4 and 2.5. A resolution of basic objects is built by defining a suitable family of equivariant functions $f_{B}$. In this section we will introduce the two main invariants used to construct these functions.

6.1. The function ord. Let $B=(W,(J, b), E)$ be a $d$-dimensional basic object. Then define the function

$$
\begin{aligned}
\operatorname{ord}_{B}^{d}: \operatorname{Sing}(J, b) & \rightarrow \mathbb{Q} \\
x & \longrightarrow \frac{\nu_{J}(x)}{b},
\end{aligned}
$$

where $\nu_{J}(x)$ denotes the order of the ideal $J \mathcal{O}_{W, x}$ at $\mathcal{O}_{W, x}$. Note that $\operatorname{ord}_{B}^{d}$ is an upper semi-continuous function with values in $\mathbb{Q}$. We will use the notation ord when the basic object is understood. 
6.2. The function $n$. Let $B=(W,(J, b), E)$ be a $d$-dimensional basic object, and let $E=\left\{H_{1}, \ldots, H_{l}\right\}$. Define

$$
\begin{aligned}
n_{B}^{d}: \operatorname{Sing}(J, b) & \rightarrow \mathbb{N} \\
x & \longrightarrow n(x)=\sharp\left\{H_{i} \subset E: x \in H_{i}\right\} .
\end{aligned}
$$

Note that $n_{B}^{d}$ is an upper semi-continuous function with values in $\mathbb{N}$. We will use the notation $n$ when the basic object is understood.

Now we want to study some of the properties of these functions, for instance, equivariance. To do so, we first need to introduce some auxiliary definitions:

6.3. The class of extendable transformations. Given a basic object $(W,(J, b), E)$ and an open set $U \subset W$, consider the restriction of $(W,(J, b), E)$ to $U,\left(U,(A, b), E_{U}\right)$.

Each sequence of transformations of basic objects over $(W,(J, b), E)$,

$$
(W,(J, b), E) \longleftarrow\left(W_{1},\left(J_{1}, b\right), E_{1}\right) \longleftarrow \ldots \longleftarrow\left(W_{k},\left(J_{k}, b\right), E_{k}\right),
$$

induces a sequence of transformations of basic objects over $\left(U,(A, b), E_{U}\right)$,

$$
\left(U,(A, b), E_{U}\right) \longleftarrow\left(U_{1},\left(A_{1}, b\right), E_{U_{1}}\right) \longleftarrow \ldots \longleftarrow\left(U_{k},\left(A_{k}, b\right), E_{U_{k}}\right),
$$

where each $U_{i}$ is an open subset of $W_{i}$, and $\left(U_{i},\left(A_{i}, b\right), E_{U_{i}}\right)$ is the restriction of $\left(W_{i},\left(J_{i}, b\right), E_{i}\right)$ to $U_{i}$. In fact, sequence (6.3.2) can be defined by setting $U_{i}$ as the pull-back of $U \subset W$ via $W \longleftarrow W_{i}$ for each index $i$ in sequence (6.3.1), and by taking the corresponding restriction to $U_{i}$. It is natural to ask if the converse holds:

Given a sequence as (6.3.2), is there a sequence as (6.3.1) which is a natural extension of the first?.

The answer is no. In fact if we consider a transformation on $\left(U,(A, b), E_{U}\right)$ as in Definition 3.6 with a smooth center $Y \subset U$, it might occur that the closure of $Y$ in $W, \bar{Y} \subset W$, is no longer smooth. However, if we consider transformations over $\left(U,(A, b), E_{U}\right)$ as the ones introduced in 3.12, then the extension can be obtained in a very natural way.

Definition 6.4. Let $B=(W,(J, b), E)$ be a basic object and let $x_{0} \in \operatorname{Sing}(J, b)$ be a point. We say that sequence (6.3.1) is $x_{0}$-extendable if and only if the following condition holds:

"Whenever $\left(W_{i},\left(J_{i}, b\right), E_{i}\right) \longleftarrow\left(W_{i+1},\left(J_{i+1}, b\right), E_{i+1}\right)$ is a permissible transformation with center $Y_{i} \subset W_{i}$ as in 3.6, the center $Y_{i}$ is mapped to $x_{0} \in W$ via $W_{i} \rightarrow W$."

We will denote by $C_{x_{0}}(B)$ the class of all $x_{0}$-extendable sequences over $(W,(J, b), E)$. 
Remark 6.5. The main property of $x_{0}$-extendable sequences can be stated as follows: Let $\left(U,(A, b), E_{U}\right)$ be the restriction of $(W,(J, b), E)$ to $U$. Assume now that $x_{0} \in \operatorname{Sing}(A, b)$, and that the sequence (6.3.2) is $x_{0}$-extendable. Then condition of Definition 6.4 guarantees that we can always extend sequence (6.3.2) to a sequence (6.3.1) so that the former is a restriction of the later.

In particular note that if sequence (6.3.1) is $x_{0}$-extendable, setting $U=$ $W \backslash\left\{x_{0}\right\}$, the restricted sequence (6.3.2) induces by composition the transformation,

$$
\left(U,(A, b), E_{U}\right) \longleftarrow\left(U_{k},\left(A_{k}, b\right),\left(E_{k}\right)_{U}\right)
$$

which is either the identity map, or a composition of projections as in 3.12.

Remark 6.6. Let $\Theta: B=(W,(J, b), E) \rightarrow B^{\prime}=\left(W^{\prime},\left(J^{\prime}, b^{\prime}\right), E^{\prime}\right)$ be an isomorphism of basic objects. Fix $x_{0} \in \operatorname{Sing}(J, b)$ and set $x_{0}^{\prime}=\Theta_{0}\left(x_{0}\right)$. It is easy to check that $\Theta$ defines a natural bijection

$$
\alpha_{\Theta}: C_{x_{0}}(B) \rightarrow C_{x_{0}^{\prime}}\left(B^{\prime}\right) .
$$

Lemma 6.7. The upper semi-continuous functions $\operatorname{ord}_{B}^{d}$ and $n_{B}^{d}$ verify properties (e) and ( $f$ ) of Definition 5.5.

Proof: Clearly if $\left(U,(A, b), E_{U}\right)$ is the restriction of $\mathcal{B}=(W,(J, b), E)$ to an open set $U$, and $x_{0} \in \operatorname{Sing}(A, b)$, the values of both functions at $x_{0}$ are independent of $U$. This shows that both $\operatorname{ord}_{\mathcal{B}}^{d}$ and $n_{\mathcal{B}}^{d}$ satisfy property (e) of Definition 5.5.

The property of equivariance of the family of functions $n_{B}^{d}$ follows from the way isomorphisms of pairs and basic objects are defined (see Definitions 4.1 and 4.6).

The property of equivariance of the functions ord is a key point in our development, and the proof is based on a very elementary and enlightening idea which we present below (see Appendix 21 for a short and formal proof of this fact):

Let $B=\left(W_{0},\left(J_{0}, b\right), E_{0}\right)$ be a basic object and consider a sequence of transformations of basic objects,

$$
\underset{\cup}{\left(W_{0}, E_{0}\right)} \longleftarrow \quad \underset{\cup}{\left(W_{1}, E_{1}\right)} \longleftarrow \cdots \underset{ }{\cup} \underset{F_{0}=\operatorname{Sing}\left(J_{0}, b\right)}{F_{1}=\operatorname{Sing}\left(J_{1}, b\right)} \quad \cdots \quad F_{k}=\underset{\operatorname{Sing}\left(J_{k}, b\right) .}{\cup}
$$

Now fix a point $x_{0} \in \operatorname{Sing}\left(J_{0}, b\right) \subset W_{0}$. Note that the morphisms $W_{k} \rightarrow W_{0}$ maps $\operatorname{Sing}\left(J_{i}, b\right) \rightarrow \operatorname{Sing}\left(J_{0}, b\right)$, and hence defines a fiber $\operatorname{Sing}\left(J_{i}, b\right)_{x_{0}} \subset$ $\operatorname{Sing}\left(J_{i}, b\right)$ over $x_{0}$. The point is that the rational number $\operatorname{ord}^{d}\left(x_{0}\right)$ can be defined in terms of: 
1. The dimension of $W$, say $d$.

2. The dimension of the fibers $\operatorname{Sing}\left(J_{i}, b\right)_{x_{0}}$, whenever sequence (6.7.1) runs over the $x_{0}$-extendable sequences (sequences in $C_{x_{0}}(B)$ ).

This already proves that the family of functions $\operatorname{ord}^{d}$ is equivariant: Fix an isomorphism $\Theta: B=(W,(J, b), E) \rightarrow B^{\prime}=\left(W^{\prime},\left(J^{\prime}, b^{\prime}\right), E^{\prime}\right)$, and let $x_{0}^{\prime}=\Theta_{0}\left(x_{0}\right) \in \operatorname{Sing}\left(J^{\prime}, b^{\prime}\right)$. By Definition 4.6 (ii) a sequence of transformations over $(W,(J, b), E)$ defines, via $\Theta$, a sequence over $\left(W^{\prime},\left(J^{\prime}, b^{\prime}\right), E^{\prime}\right)$. By Remark 6.6, this correspondence maps $x_{0}$-extendable sequences into $x_{0}^{\prime}$ extendable sequences of transformations defining the bijection $\alpha_{\Theta}: C_{x_{0}}(B) \rightarrow$ $C_{x_{0}^{\prime}}\left(B^{\prime}\right)$ (see Remark 6.6). Each isomorphism $\Theta_{i}: \operatorname{Sing}\left(J_{i}, b\right) \cong \operatorname{Sing}\left(J_{i}^{\prime}, b^{\prime}\right)$ maps fibers to fibers,

$$
\operatorname{Sing}\left(J_{i}, b\right)_{x_{0}} \cong \operatorname{Sing}\left(J_{i}^{\prime}, b^{\prime}\right)_{x_{0}^{\prime}}
$$

so both fibers have the same dimensions, and hence the rational numbers $\operatorname{ord}_{B}^{d}\left(x_{0}\right)$ will coincide with $\operatorname{ord}_{B^{\prime}}^{d}\left(x_{0}^{\prime}\right)$.

Remark 6.8. Since the functions $f_{i}(x)$ which appear in Definition 5.5 will be defined in terms of the functions $\operatorname{ord}_{B}^{d}$ and $n_{B}^{d}$, they will ultimately inherit the properties of equivariance required in Definition 5.5 (e) and compatibility with open restrictions in $5.6(2)$.

Remark 6.9. The notion of transformation of basic object defined in 3.12 is introduced precisely in order to ensure that $\operatorname{ord}_{B}^{d}$ is an equivariant family of functions. As an example to illustrate this fact, let

$$
W=\mathbb{A}_{k}^{2}=\operatorname{Spec}(k[x, y]),
$$

and set $J=<x, y>$. If transformations as the ones described in 3.12 were not introduced, then the identity would induce an isomorphism of basic objects

$$
I d: B=\left(W,\left(J^{5}, 4\right), \emptyset\right) \cong B^{\prime}=\left(W,\left(J^{5}, 5\right), \emptyset\right) .
$$

Note that for both basic objects the singular locus is the origin $(0,0)$, and that both are resolvable by one quadratic transformation. Note also that those would be the only transformations of basic objects if only transformations as the ones introduced in 3.6 were allowed. Now

$$
\operatorname{ord}_{B}^{2}((0,0))=5 / 4 \quad \text { and } \quad \operatorname{ord}_{B^{\prime}}^{2}((0,0))=1,
$$

so the family of functions $\operatorname{ord}_{B}^{d}$ would not be compatible with the identity. The law of transformation introduced in 3.12 enlarges the class of all possible transformations, and therefore makes the notion of isomorphism of basic objects stronger. In the case of our example, it turns out that the identity map is not an isomorphism of basic objects when adding 3.12 as possible law of transformation. 


\section{Part III. Applications}

\section{Weak and strict transforms of ideals: Strong Factor- izing Desingularization}

In this section we discuss the difference between the notions of weak and strict transforms of ideals. As a related matter we establish Theorem 7.3 which says that given a variety we can resolve its singularities, and in addition require a very natural algebraic condition on the lifting of the ideal defining the variety (see Theorem 7.3 (iii)). We finish this section by presenting some applications of this result.

Definition 7.1. Let $J \subset \mathcal{O}_{W}$ be a non-zero sheaf of ideals, and let

$$
W \leftarrow W_{1} \leftarrow \ldots \leftarrow W_{k}
$$

be a sequence of blowing-ups at regular centers. Then for each $i \in\{1, \ldots, k\}$ there is an intrinsic factorization

$$
J \mathcal{O}_{W_{i}}=\mathcal{L}_{i} \bar{J}_{i}
$$

where:

(a) The sheaf of ideals $\mathcal{L}_{i}$ is locally principal and supported on the exceptional locus.

(b) The sheaf of ideals $\bar{J}_{i}$ has no height one exceptional components: All codimension one components of $V\left(\bar{J}_{i}\right)$ are strict transforms of the codimension one components of $V(J)$.

With this notation, for $i \in\{1, \ldots, k\}, \bar{J}_{i}$ is the weak transform of $J$ in $W_{i}$, $J_{i}=J \mathcal{O}_{W_{i}}$ is the total transform of $J$ in $W_{i}$, and the strict transform of $J$ in $W_{i}, \widetilde{J}_{i}$, is defined by

$$
\widetilde{J}_{i}=\bigcup_{j=1}^{\infty}\left(\widetilde{J}_{i-1} \mathcal{O}_{W_{j}}:_{\mathcal{O}_{W_{i}}} \mathcal{I}\left(H_{i}\right)^{j}\right),
$$

where $H_{i}$ denotes the exceptional divisor in $W_{i-1} \leftarrow W_{i}$. Therefore we have the inclusions,

$$
J_{i} \subset \bar{J}_{i} \subset \widetilde{J}_{i}
$$

for $i=1, \ldots, r$. 
In general these are strict inclusions, i.e., the notions of weak and strict transforms of ideals are different: For instance, let $X \subset W$ be a reduced subscheme and let $\pi_{r}: W_{r} \longrightarrow W$ be an embedded desingularization of $X$. Then

$$
\mathcal{I}(X) \mathcal{O}_{W_{r}}=\mathcal{L}_{r} \cdot \bar{J}_{r}
$$

and unless $X$ is a hypersurface, usually $\bar{J}_{r}$ does not coincide with $\mathcal{I}\left(X_{r}\right)=$ $\widetilde{J}_{r}$, the ideal of the strict transform of $X$ in $W_{r}$. The reason is that, during the process of desingularization, new primary components of $\bar{J}_{r}$ arise. This can clearly be seen by examining the following example:

Example 7.2. Let $W$ be the real affine space, and let $G$ be the curve parameterized by

$$
\left(t^{6}, t^{9}, t^{13}\right) \text {. }
$$

This curve is defined by the ideal

$$
\mathcal{I}(G)=\left\langle x^{3}-y^{2}, x^{2} y^{3}-z^{3}\right\rangle .
$$

Note that $G$ is minimally embedded in $\mathbb{A}_{\mathbb{Q}}^{3}$ at the origin $(\mathbf{0}, \mathbf{0}, \mathbf{0})$, and that this is the only singular point of the curve. Let

$$
T=V\left(\left\langle x^{3}-y^{2}\right\rangle\right)
$$

Since the origin is the only singular point of $G$, to obtain a desingularization by monoidal transformations we have to blow-up this point: $\pi_{1}: W_{1} \longrightarrow W$.

Note that

$$
\mathcal{I}(G) \mathcal{O}_{W_{1}}=\mathcal{I}\left(H_{1}\right)^{2} \bar{J}_{1}
$$

where $H_{1} \subset W_{1}$ is the exceptional divisor and $\bar{J}_{1}$ the weak transform of $\mathcal{I}(G)$ in $\mathcal{O}_{W_{1}}$. Let $T_{1} \subset W_{1}$ be the strict transform of $T$ in $W_{1}$. Note that $\mathcal{I}\left(T_{1}\right) \subset \bar{J}_{1}$.

It is easy to see that $\bar{J}_{1}$ has an embedded component corresponding to the line

$$
L=H_{1} \cap T_{1} \subset V\left(\bar{J}_{1}\right)
$$

We will never eliminate this primary component $\mathcal{I}(L)$ of $\bar{J}_{1}$ by blowingup at points of the strict transform of $G$. 


\section{A Strong Factorizing Desingularization Theorem}

In [13] we show that, given a reduced subscheme, $X \subset W$, it is possible to construct a finite sequence of monoidal transformations,

$$
W \longleftarrow W_{1} \longleftarrow \ldots \longleftarrow W_{r}
$$

so that $X_{r}$ is non-singular, and the weak transform of $J=\mathcal{I}(X)$ in $W_{r}$ describes the strict transform of $X$ in $W_{r}$, i.e. that $\bar{J}_{r}$ coincides with $\mathcal{I}\left(X_{r}\right)$. That is, we present an algorithm of desingularization which provides in a simple manner the equations describing the desingularization of $X$. An announcement of this result has appeared in [14].

In fact, our statement is valid under milder hypothesis; for instance, we do not need to assume that $X$ is reduced. The theorem can be stated more precisely as follows:

7.3. A Strong Factorizing Desingularization Theorem. [13, Theorem 1.2] Let $\left(W_{0}, E_{0}=\{\emptyset\}\right)$ be a pair and let $X=X_{0} \subset W_{0}$ be a closed subscheme defined by $\mathcal{I}\left(X_{0}\right) \subset \mathcal{O}_{W_{0}}$. Assume that the open set of regular points $\operatorname{Reg}(X)$ is dense in $X$. Then there exists a finite sequence of transformations of pairs,

$$
\left(W_{0}, E_{0}\right) \longleftarrow \ldots \longleftarrow\left(W_{r}, E_{r}\right)
$$

inducing a proper birational morphism $\pi_{r}: W_{r} \longrightarrow W_{0}$, so that setting $E_{r}=\left\{H_{1}, \ldots, H_{r}\right\}$, and letting $X_{r} \subset W_{r}$ be the strict transform of $X_{0}$, we have that:

(i) $X_{r}$ is regular in $W_{r}$, and $W_{r} \backslash \cup_{i=1}^{r} H_{i} \simeq W_{0} \backslash \operatorname{Sing}(X)$. In particular $\operatorname{Reg}(X) \cong \pi_{r}^{-1}(\operatorname{Reg}(X)) \subset X_{r}$ (via $\pi_{r}$ restricted to $\left.X_{r}\right)$.

(ii) $X_{r}$ has normal crossings with $E_{r}=\cup_{i=1}^{r} H_{i}$ (the exceptional locus of $\pi_{r}$ ).

(iii) The total transform of the ideal $\mathcal{I}\left(X_{0}\right) \subset \mathcal{O}_{W_{0}}$ factors as a product of ideals in $\mathcal{O}_{W_{r}}$ :

$$
\mathcal{I}(X) \mathcal{O}_{W_{r}}=\mathcal{L} \cdot \mathcal{I}\left(X_{r}\right),
$$

where now $\mathcal{I}\left(X_{r}\right) \subset \mathcal{O}_{W_{r}}$ denotes the sheaf of ideals defining $X_{r}$, and

$$
\mathcal{L}=\mathcal{I}\left(H_{1}\right)^{a_{1}} \cdot \ldots \cdot \mathcal{I}\left(H_{r}\right)^{a_{r}}
$$

is an invertible sheaf of ideals supported on the exceptional locus of $\pi_{r}$.

Parts (i) and (ii) are the classical statement of the theorem of resolution of singularities. However, part (iii) is new (see 7.4 below). Note also that (i) ensures that, in our algorithm, the only points of $W_{0}$ that will be modified by the morphism $\pi_{r}$ are the ones in $\operatorname{Sing}\left(X_{0}\right)$. 
7.4. Why is part (iii) new? [13, Section 2] In Hironaka's line of proof, the centers of monoidal transformations, chosen in accordance with the so called standard basis, are always included in the strict transform of the scheme. For instance, in Example 7.2, the first monoidal transformation must be the the blowing-up at the origin, and any other center will also have dimension zero. The one dimensional component which appears after this blowing-up, $H_{1} \cap T_{1}$ (see (7.2.2)), is a primary component of $\bar{J}_{1}$, and will never be eliminated by blowing-up at centers supported on the singular locus of $G$. Hence (iii) will never hold for desingularizations of this curve that follow from Hironaka's proof.

In order to achieve (iii) one must blow-up $H_{1} \cap T_{1}$ (or some strict transform of it). The new algorithm that we propose, first considers the quadratic transformation $\pi: W_{1} \longrightarrow W_{0}$, and, some steps later, the blowing-up at the strict transform of the one dimensional scheme $H_{1} \cap T_{1}$. Since $H_{1} \cap T_{1}$ is mapped to the singular locus, the first isomorphism in Theorem 7.3 (i) is preserved after such monoidal transformation.

We think of a subscheme $X$ of a smooth scheme $W$, at least locally, as a finite number of equations defining the ideal $\mathcal{I}(X)$. An algorithm of desingularization should provide us with:

(1) A sequence of monoidal transformations over the smooth scheme $W$,

$$
W_{0}=W \leftarrow W_{1} \leftarrow \ldots \leftarrow W_{n}
$$

so that conditions (i) and (ii) Theorem 7.3 hold for the strict transform of $X$ at $W_{n}$.

(2) A pattern of manipulation of equations defining $X$, so as to obtain, at least locally at an open covering of $W_{n}$, equations defining the strict transform $X_{n}$ of $X$ at $W_{n}$.

So (2) indicates how the original equations defining $X$ have to be treated, at an affine open subset of $W_{n}$, in order to obtain local equations defining $X_{n}$. While this is very complicated in Hironaka's line of proof, here it is a direct consequence of Theorem 7.3 (iii). In fact, for algorithms that follow Hironaka's proof, to get both (1) and (2) one must consider the strict transform of the ideal of the subscheme at each monoidal transformation. In that setting one has to choose a standard basis of the ideal, which is a system of generators of the ideal of the subscheme, but such choice of generators must be changed if the maximum Hilbert Samuel invariant drops in the sequence of monoidal transformations. All of these complications are avoided in our proof, which simplifies both (1) and (2). 
The idea of the proof of Theorem 7.3:

The notion of "excess of embedding codimension" of an ideal

The idea behind the proof of Theorem 7.3 is to construct a sequence of monoidal transformations

$$
W \leftarrow W_{1} \leftarrow \ldots \leftarrow W_{r},
$$

so that the exceptional components of the total transform of $J$ in $W_{r}, J \mathcal{O}_{W_{r}}$, become locally principal.

Thus, the strategy is to show first that for a suitable sequence of monoidal transformations

$$
W_{0} \leftarrow W_{1} \leftarrow \ldots \leftarrow W_{l},
$$

the subscheme $V\left(\left(\bar{J}_{l}\right)_{y}\right)$ is locally included in a smooth hypersurface, and this condition holds at every $y \in V\left(\bar{J}_{l}\right)$. Then we show that by applying more monoidal transformations

$$
W_{l} \leftarrow W_{l+1} \leftarrow \ldots \leftarrow W_{m},
$$

the closed subscheme $V\left(\left(\bar{J}_{m}\right)_{y}\right)$ is locally included in a smooth subscheme of codimension two, for every $y \in V\left(\bar{J}_{m}\right)$. To obtain our result we repeat this process as many times as needed.

This strategy leads us to the notion of excess of embedding codimension of an ideal:

Definition 7.5. [13, Definition 5.2] Let $(W, E)$ be a pair, and let $J \subset \mathcal{O}_{W}$ be a non-zero sheaf of ideals.

(a) We say that $J$ has excess of embedding codimension $\geq a$ in $(W, E)$ at a point $y \in W$, if either $J_{y}=\mathcal{O}_{W, y}$, or there is a regular system of parameters $\left\{x_{1}, x_{2}, \ldots, x_{d}\right\} \subset \mathcal{O}_{W, y}$ such that

(i) $<x_{1}, x_{2}, \ldots, x_{a}>\subset J_{y} \subset \mathcal{O}_{W, y}$, and

(ii) every hypersurface $H_{i} \in E$ containing the point $y$ has a local equation

$$
\mathcal{I}\left(H_{i}\right)=<x_{i_{j}}>\subset \mathcal{O}_{W, y}
$$

with $i_{j}>a$.

(b) We say that $J$ has excess of embedding codimension $\geq a$ in $(W, E)$, if the conditions stated in (a) hold at every point $y \in W$. We will abbreviate this by saying that $J$ has excess of codimension $\geq a$ in $(W, E)$, or $(W, E)$-codimension $\geq a$. Reference to the pair $(W, E)$ will be omitted if it is clear from the context. 
Remark 7.6. Note that any non-zero ideal $I \subset \mathcal{O}_{W}$ has excess codimension $\geq 0$ in $(W, E)$, since such condition is vacuously satisfied in that case.

Remark 7.7. If $X \subset W$ is a regular subscheme of pure codimension $e$, then the sheaf of ideals $\mathcal{I}(X) \subset \mathcal{O}_{W}$ has excess of codimension $\geq e$ in $(W, E=\emptyset)$.

The main result over which the proof of Theorem 7.3 is based is [13, Lemma 4.7]. What we state below is a slightly stronger version of this lemma:

Lemma 7.8. Let $(W, E)$ be a pair, and let $J$ be a non-zero sheaf of ideals. Assume that there is a non-empty open set

$$
U^{a} \subset W \backslash E \quad \text { such that } \quad V(J) \cap U^{a} \neq \emptyset
$$

and that $J$ has $(W, E)$-excess of codimension $\geq$ a at any point of $U^{a}$. Then there is a finite sequence of transformation of pairs:

$$
\left(W_{k}, E_{k}\right) \longrightarrow \ldots \longrightarrow\left(W_{1}, E_{1}\right) \longrightarrow\left(W_{0}, E_{0}\right)=(W, E)
$$

such that if

$$
J \mathcal{O}_{W_{k}}=\mathcal{L}_{k} \bar{J}_{k}
$$

then:

(i) The weak transform of $J$ in $W_{k}, \bar{J}_{k}$, has $\left(W_{k}, E_{k}\right)$-excess of codimension $\geq a$.

(ii) The birational morphism $W_{k} \longrightarrow W$ induces an isomorphism over the open set $U^{a} \subset W$.

(iii) If in addition we can express $E=\left\{H_{1}, \ldots, H_{k}\right\}$ as a disjoint union, $E=E^{a} \sqcup E^{a^{\prime}}$, so that $J$ has excess of embedding codimension $\geq$ a at any point of $V(J) \cap E^{a}$, then the sequence (7.8.1) can be constructed so that (ii) also holds if we only assume that

$$
U^{a} \subset W \backslash E^{a^{\prime}}
$$

Proof: Parts (i) and (ii) have been proven in [13]. For part (iii) it is enough to observe that if we set

$$
E_{0}^{+}=E^{a} \quad \text { and } \quad E_{0}^{-}=E^{a^{\prime}},
$$

then the morphism $W_{k} \longrightarrow W$ induces an isomorphism on $E \backslash E^{a^{\prime}}$ (see Definition 15.13, where $E_{0}^{+}$and $E_{0}^{-}$are introduced, and see also [13, Definition 5.9]). 
The following is an easy consequence of Lemma 7.8:

Corollary 7.9. Fix a pair $(W, E)$. Let $X \subset W$ be a closed subscheme, let $J=\mathcal{I}(X)$, and let

$$
X=X(1) \cup X(2) \cup \ldots \cup X(e),
$$

where for $i=1, \ldots, e$, each $X(i)$ is a closed subscheme of pure codimension $i \leq n$. Assume that $\operatorname{Reg}(X(e)) \neq \emptyset$ and let $Z$ be the closure of $\operatorname{Reg}(X(e))$ in $W$. Suppose that $E=E^{e} \sqcup E^{e^{\prime}}$ and that $Z$ has normal crossings with $E^{e}$ at any point of $Z \cap E^{e}$ ( $E^{e}$ might be empty). Let $\mathcal{Q} \subset W$ be the open set $W \backslash\left[X(1) \cup \ldots \cup X(e-1) \cup \operatorname{Sing}(X(e)) \cup E^{e^{\prime}}\right]$. Then there is a finite sequence of transformations of pairs,

$$
\left(W_{k}, E_{k}\right) \longrightarrow \ldots \longrightarrow\left(W_{1}, E_{1}\right) \longrightarrow\left(W_{0}, E_{0}\right)=(W, E)
$$

such that:

(i) The strict transform of $Z$ in $W_{k}, Z_{k}$, is regular, and has normal crossings with $E_{k}$.

(ii) The morphism $W_{k} \rightarrow W$ induces an isomorphism on $\mathcal{Q}$.

(iii) The weak transform of $J$ in $W_{k}, \bar{J}_{k}$, coincides with $\mathcal{I}\left(Z_{k}\right)$, where $Z_{k}$ is the strict transform of $Z$ in $W_{k}$, i.e.

$$
J \mathcal{O}_{W_{k}}=\mathcal{L}_{k} \mathcal{I}\left(Z_{k}\right),
$$

where $\mathcal{L}_{k}$ is a locally principal sheaf of ideals supported on the exceptional locus of $W_{k} \rightarrow W$.

Proof: By hypothesis, $J$ has $(W, E)$-excess of embedding codimension $\geq e$ in $\mathcal{Q}$. Then by Lemma 7.8, there is a finite sequence of transformations of pairs,

$$
\left(W_{k}, E_{k}\right) \longrightarrow \ldots \longrightarrow\left(W_{1}, E_{1}\right) \longrightarrow\left(W_{0}, E_{0}\right)=(W, E)
$$

such that:

(a) The weak transform of $J$ in $W_{k}, \bar{J}_{k}$, has excess of codimension $e$ in $\left(W_{k}, E_{k}\right)$.

(b) The morphism $W_{k} \rightarrow W$ induces an isomorphism on $\mathcal{Q}$.

Now write

$$
V\left(\bar{J}_{k}\right)=Y_{1} \cup \ldots \cup Y_{m}
$$

where each $Y_{i}$ is an irreducible component of codimension at least $e$. Since $Z_{k} \subset V\left(\bar{J}_{k}\right)$, without lost of generality, we may assume that

$$
Z_{k}=Y_{1} \cup \ldots \cup Y_{s},
$$

where $1 \leq s \leq m$. 
Note that since $\bar{J}_{k}$ has excess of embedding codimension $\geq e$ in $\left(W_{k}, E_{k}\right)$, $Z_{k}=Y_{1} \cup \ldots \cup Y_{s}$ is regular. Now, if $s=m$ we are done. On the other hand, if $s<m$, then $V\left(\bar{J}_{k}\right)$ is a disjoint union of $Y_{1} \cup \ldots \cup Y_{s}$ and $Y_{s+1} \cup \ldots \cup Y_{m}$, i.e.,

$$
V\left(\bar{J}_{k}\right)=\left[Y_{1} \cup \ldots \cup Y_{s}\right] \sqcup\left[Y_{s+1} \cup \ldots \cup Y_{m}\right]
$$

(here we use the fact that $Z_{k}=Y_{1} \cup \ldots \cup Y_{s}$ is regular and has codimension $e$ : If (7.9.3) were not a disjoint union, at any point of the intersection the codimension would be greater than $e$ ). As a consequence

$$
\bar{J}_{k}=\mathcal{I}\left(Y_{1} \cup \ldots \cup Y_{s}\right) \cdot \mathcal{I}\left(Y_{s+1} \cup \ldots \cup Y_{m}\right) \text {. }
$$

By Theorem 2.5, there is an enlargement of sequence 7.9.2,

$$
\left(W_{k}, E_{k}\right) \leftarrow \ldots \leftarrow\left(W_{l}, E_{l}\right)
$$

which defines a strong principalization of $\mathcal{I}\left(Y_{s+1} \cup \ldots \cup Y_{m}\right) \subset \mathcal{O}_{W_{k}}$. Therefore

$$
J \mathcal{O}_{W_{l}}=\mathcal{L}_{l} \bar{J}_{l}
$$

and

$$
\bar{J}_{l}=\mathcal{I}\left(Z_{l}\right)
$$

Note that since (7.9.3) is a disjoint union, $W_{l} \rightarrow W$ defines an isomorphism on $\mathcal{Q}$.

\section{An application of Corollary 7.9: Log resolutions of divisors}

Definition 7.10. Let $D \subset W$ be a divisor. A log resolution of the pair $(W, D)$ is a proper and birational morphism,

$$
\mu: W^{\prime} \rightarrow W
$$

such that $W^{\prime}$ is non-singular and $\mu^{*}(D)+\operatorname{Exc}(\mu)$ is a normal crossing divisor.

As an application of Corollary 7.9 we prove the following statement:

Theorem 7.11. Let $D=\sum_{i=1}^{r} a_{i} D_{i} \subset W$ be a divisor, and let $V \subset W$ be the open set of points where $D$ has normal crossings. Then there is a log resolution of $D$

$$
\mu: W^{\prime} \rightarrow W
$$

which induces an isomorphism on $V$. 
7.12. The motivation. The interest of this result, lies in its role in proving statements which involve the application of vanishing theorems in the compactification of a given variety. More explicitly, assume that $W$ is a smooth quasi-projective variety and that $D \subset W$ is a normal crossing divisor. Some vanishing theorems are only stated for projective varieties. Hence, if $W$ is not projective, there is a compactification of $W, \bar{W}$, where the vanishing theorems hold. However, it may happen that the compactification of $D$ in $\bar{W}, \bar{D}$, is not a normal crossing divisor any more. By applying a log resolution to $(\bar{W}, \bar{D})$,

$$
\bar{\mu}: \bar{W}^{\prime} \rightarrow \bar{W}
$$

we may assume that

$$
\bar{\mu}^{*}(\bar{D})
$$

is a divisor with normal crossings. For technical reasons, it may be useful to assume that the induced morphism

$$
\mu: W^{\prime} \rightarrow W
$$

is an isomorphism over $D \subset W$. To this end, it would be enough if

$$
\bar{\mu}: \bar{W}^{\prime} \rightarrow \bar{W}
$$

is an isomorphism over the points where $\bar{D}$ is already a normal crossing divisor. For a concrete example see [25].

Before proving Theorem 7.11 we will introduce some notation and prove some auxiliary results (Proposition 7.16). The proof of Theorem 7.11, which is given in 7.17, will follow from Proposition 7.16 and an inductive argument.

Remark 7.13. In what follows, we will denote by $D=\sum_{i=1}^{r} a_{i} D_{i}$ a divisor in $W$, where each $D_{i}$ corresponds to an irreducible and reduced hypersurface in $W$. Let $J=\mathcal{O}_{W}\left(-D_{1}\right)+\ldots+\mathcal{O}_{W}\left(-D_{r}\right)$ be the ideal corresponding to the intersection of all the components of $D$. If

$$
W_{k} \rightarrow W_{k-1} \rightarrow \ldots \rightarrow W_{1} \longrightarrow W
$$

is a finite sequence of monoidal transformations, and if $\pi_{k}: W_{k} \rightarrow W$ denotes the composition, then we will write

$$
\pi_{k}^{*}(D)=\sum_{i=1}^{r} a_{i} D_{i}^{[k]}+F_{k}=D^{[k]}+F_{k},
$$

where $D_{i}^{[k]} \subset W_{k}$ denotes the strict transform of $D_{i}$ in $W_{k}$ for $i=1, \ldots, r$, and $F_{k}$ is a divisor supported in the exceptional locus. Let $J^{[k]}$ be the sheaf 
of ideals $\mathcal{O}_{W_{k}}\left(-D_{1}^{[k]}\right)+\ldots+\mathcal{O}_{W_{k}}\left(-D_{r}^{[k]}\right)$ which describes the intersection $D_{1}^{[k]} \cap \ldots \cap D_{r}^{[k]}$ in $W_{k}$. Note that if $\bar{J}_{k}$ is the weak transform of $J=$ $\mathcal{O}_{W}\left(-D_{1}\right)+\ldots+\mathcal{O}_{W}\left(-D_{r}\right)$ in $\mathcal{O}_{W_{k}}$, then

$$
\bar{J}_{k} \subset J^{[k]} .
$$

This containment can be proven by an inductive argument: Let $C \subset W$ be a center such that $\nu_{C}\left(\mathcal{O}_{W}\left(-D_{i}\right)\right)=a_{i}$, for $i=1, \ldots, r\left(\right.$ here $\nu_{C}\left(\mathcal{O}_{W}\left(-D_{i}\right)\right)$ denotes the order of the ideal $\mathcal{O}\left(-D_{i}\right)$ at $\left.C\right)$. Then $\nu_{C}(J)=b=\operatorname{Min}\left\{a_{1}, \ldots, a_{r}\right\}$. Let $W \leftarrow W_{1}$ be the monoidal transformation with center $C$. Then, if $H_{1}$ denotes the exceptional divisor,

$$
\bar{J}_{1}=\left(J \mathcal{O}_{W_{1}}: \mathcal{I}\left(H_{1}\right)^{b}\right) \quad \text { and } \quad J^{[1]}=\mathcal{O}_{W_{1}}\left(-D_{1}^{[1]}\right)+\ldots+\mathcal{O}_{W_{1}}\left(-D_{r}^{[1]}\right),
$$

therefore,

$$
\bar{J}_{1} \subset J^{[1]} \quad \text { and } \quad \bar{J}_{1}=\mathcal{M}_{1,1} \mathcal{O}_{W_{1}}\left(-D_{1}^{[1]}\right)+\ldots+\mathcal{M}_{r, 1} \mathcal{O}_{W_{1}}\left(-D_{r}^{[1]}\right),
$$

for some ideals $\mathcal{M}_{i, 1} \subset \mathcal{O}_{W_{1}}, i=1, \ldots, r$.

Now, assume by inductive hypothesis that after a finite sequence of blowing-ups,

$$
W \leftarrow W_{1} \leftarrow \ldots \leftarrow W_{k}
$$

at smooth centers $C_{i} \subset \bar{J}_{i}, i=1, \ldots, k-1$, we have that

$$
\bar{J}_{k} \subset J^{[k]} \quad \text { and } \quad \bar{J}_{k}=\mathcal{M}_{1, k} \mathcal{O}_{W_{k}}\left(-D_{1}^{[k]}\right)+\ldots+\mathcal{M}_{r, k} \mathcal{O}_{W_{k}}\left(-D_{r}^{[k]}\right) \text {, }
$$

for some ideals $\mathcal{M}_{i, k} \subset \mathcal{O}_{W_{k}}$.

Let $C_{k} \subset V\left(\bar{J}_{k}\right) \subset W_{k}$ be a smooth center, assume that $\nu_{C_{k}}\left(\mathcal{O}_{W_{k}}\left(-D_{i}^{[k]}\right)\right)=$ $a_{i, k}$, for $i=1, \ldots, r$ and that $\nu_{C}\left(\bar{J}_{k}\right)=b_{k}$. Note that $b_{k}=\operatorname{Min}\left\{a_{1, k}+\right.$ $\left.\nu_{C_{k}}\left(\mathcal{M}_{1, k}\right), \ldots, a_{r, k}+\nu_{C_{k}}\left(\mathcal{M}_{r, k}\right)\right\}$. Then if $W_{k} \leftarrow W_{k+1}$ is the blowing-up at $C_{k}$ and if $H_{k+1}$ denotes the exceptional divisor,

$$
\begin{aligned}
\bar{J}_{k+1} & =\left(\bar{J}_{k} \mathcal{O}_{W_{k+1}}: \mathcal{I}\left(H_{k+1}\right)^{b_{k}}\right) \\
\text { and } \quad J^{[k+1]} & =\mathcal{O}_{W_{k+1}}\left(-D_{1}^{[k+1]}\right)+\ldots+\mathcal{O}_{W_{k+1}}\left(-D_{r}^{[k+1]}\right) .
\end{aligned}
$$

Now note that

$$
\begin{aligned}
\bar{J}_{k+1} & =\left(\bar{J}_{k} \mathcal{O}_{W_{k+1}}: \mathcal{I}\left(H_{k+1}\right)^{b_{k}}\right)= \\
& =\left(\left[\mathcal{M}_{1, k} \mathcal{O}_{W_{k}}\left(-D_{1}^{[k]}\right)+\ldots+\mathcal{M}_{r, k} \mathcal{O}_{W_{k}}\left(-D_{r}^{[k]}\right)\right] \mathcal{O}_{W_{k+1}}: \mathcal{I}\left(H_{k+1}\right)^{b_{k}}\right) .
\end{aligned}
$$

Hence,

$$
\begin{aligned}
\bar{J}_{k+1} & \subset J^{[k+1]}, \\
\text { and } \quad \bar{J}_{k+1} & =\mathcal{M}_{1, k+1} \mathcal{O}_{W_{k+1}}\left(-D_{1}^{[k+1]}\right)+\ldots+\mathcal{M}_{r, k+1} \mathcal{O}_{W_{k+1}}\left(-D_{r}^{[k+1]}\right),
\end{aligned}
$$

for some ideals $\mathcal{M}_{i, k+1} \subset \mathcal{O}_{W_{k+1}}, i=1, \ldots, r$. 
Definition 7.14. Let $(W, E)$ be a pair, and let $D=\sum_{i=1}^{r} a_{i} D_{i} \subset W$ be a divisor, where each $D_{i}$ is irreducible and reduced. Let $s \in\{1, \ldots, r\}$.

- We will say that $D$ has $s$-normal crossings in $(W, E)$ at a point $x \in W$ if:

(a) There are fewer than $s$ components of $D$ containing $x$; or else,

(b) The divisors $\left\{\cup_{i=1}^{r} D \cup E\right\}$ have normal crossings at $x$.

- If $D$ does not have $s$-normal crossings in $(W, E)$ at $x$ for any $s \in$ $\{1, \ldots, r\}$, then we will say that $D$ has $r+1$-normal crossings at $x$ in $(W, E)$, since this condition is always vacuously satisfied.

- If we say that $D$ has $s$-normal crossings in $(W, E)$ we will understand that it has $t$-normal crossings in $(W, E)$ at any point of $W$, with $t \geq s$.

Remark 7.15. Note that:

(i) If a divisor $D$ has normal crossings then it has normal crossings at any point of $W$ and hence it has 1 -normal crossings in $(W, \emptyset)$ at any point $x \in W$.

(ii) If $D$ has 1 -normal crossings in $(W, E)$ at any point $x \in W$, then $D$ has normal crossings, and $\{D \cup E\}$ have normal crossings.

Proposition 7.16. Let $(W, E)$ be a pair, let $D=\sum_{i=1}^{s} a_{i} D_{i}$ be a divisor, and assume that $E=E^{s} \sqcup E^{s^{\prime}}$ is a disjoint union such that $D$ has s-normal crossings at any point of $E^{s}$ ( $E^{s}$ might be empty). Let $U$ be the subset of $W \backslash E^{s^{\prime}}$ where $D$ has normal crossings, and let $U^{s}$ be the subset of $W \backslash E^{s^{\prime}}$ where $D$ has s-normal crossings. Then there is a finite sequence of transformations of pairs,

$$
\left(W_{0}, E_{0}\right)=(W, E) \leftarrow\left(W_{1}, E_{1}\right) \leftarrow \ldots \leftarrow\left(W_{l}, E_{l}\right),
$$

such that

(i) The divisor $D^{[l]}=\sum_{i=1}^{s} D_{i}^{[l]}$ has $s-$ normal crossings in $\left(W_{l}, E_{l}\right)$.

(ii) The morphism $\pi_{l}: W_{l} \rightarrow W$ induces an isomorphism on $U^{s} \subset W$ (and hence on $U$ ).

Proof: First note that by Definition 7.14 (b), $U \subset U^{s}$. We will distinguish three cases:

Case 1: If $D_{1} \cap \ldots \cap D_{s}=\emptyset$ then there is nothing to prove.

Case 2: If there are no points in $D_{1} \cap \ldots \cap D_{s}$ where $\left\{D_{1}, \ldots, D_{s}, E\right\}$ have normal crossings, or if $D_{1} \cap \ldots \cap D_{s} \subset E^{s^{\prime}}$, then applying Theorem 2.5 to 
$J=\mathcal{O}_{W}\left(-D_{1}\right)+\cdots+\mathcal{O}_{W}\left(-D_{s}\right)$, there is a finite sequence of blowing-ups at regular centers,

$$
\left(W_{0}, E_{0}\right)=(W, E) \leftarrow\left(W_{1}, E_{1}\right) \leftarrow \ldots \leftarrow\left(W_{l}, E_{l}\right),
$$

such that the weak transform of $J$ in $\mathcal{O}_{W_{l}}, \bar{J}_{l}$, is trivial. Since,

$$
\mathcal{O}_{W_{l}}=\bar{J}_{l} \subset J^{[l]},
$$

(see Remark 7.13), we have that

$$
D_{1}^{[l]} \cap \ldots \cap D_{s}^{[l]}=\emptyset .
$$

Note that in this case $\pi_{l}: W_{l} \rightarrow W$ induces an isomorphism on $W \backslash V(J)$, so in particular it induces an isomorphism on $U^{s}$ and hence on $U$.

Case 3: As in the previous case, set $J=\mathcal{O}_{W}\left(-D_{1}\right)+\ldots+\mathcal{O}_{W}\left(-D_{s}\right)$. If there is a point $x \in D_{1} \cap \ldots \cap D_{s}$ where $\left\{D_{1}, \ldots, D_{s}, E\right\}$ have normal crossings and $x \notin E^{s^{\prime}}$, then $J$ has excess of codimension $\geq s$ at $x$, and in fact $J$ has excess of codimension $\geq s$ at any point of $V(J) \cap U^{s}$. Let $Z$ be the closure of $U^{s} \cap V(J)$ in $W$. By Corollary 7.9 applied to $X=V(J)$ there is a finite sequence of transformation of pairs

$$
\left(W_{0}, E_{0}\right)=(W, E) \leftarrow\left(W_{1}, E_{1}\right) \leftarrow \ldots \leftarrow\left(W_{l}, E_{l}\right),
$$

such that:

(a) The strict transform of $Z$ in $W_{l}, Z_{l}$, is smooth and has normal crossings with $E_{l}$.

(b) The morphism $W_{l} \rightarrow W$ induces an isomorphism on $U^{s}$, and hence on $U$.

(c) The ideal $\bar{J}_{l}$ coincides with $\mathcal{I}\left(Z_{l}\right)$.

Therefore $\left\{D^{[l]}, E_{l}\right\}$ have normal crossings at any point of $Z_{l}=D_{1}^{[l]} \cap \ldots \cap D_{s}^{[l]}$.

7.17. Proof of Theorem 7.11: Assume that $D$ has $t$-normal crossings in $(W, E)$, with $1<t \leq r+1$, but that it does not have $(t-1)$-normal crossings in $(W, E)$. Let $\Sigma$ be the set of all possible $(t-1)$-tuples of elements in $\{1, \ldots, r\}$. If $\sigma \in \Sigma$, then we will denote by $D_{\sigma}$ the divisor $\sum_{i \in \sigma} D_{i}$, by $J_{\sigma}$ the ideal $\sum_{i \in \sigma} \mathcal{O}_{W}\left(-D_{i}\right)$, and by $G_{\sigma}$ the closed set of points in $V\left(J_{\sigma}\right)$ where $J_{\sigma}$ does not have excess of embedding codimension $\geq t-1$.

Fix an order in $\Sigma$ such that if

$$
\Sigma=\left\{\sigma_{1}, \ldots, \sigma_{m}\right\},
$$

with

$$
\sigma_{1}<\cdots<\sigma_{m-1}<\sigma_{m}
$$


then every divisor in

$$
\mathcal{G}=\left\{D_{\sigma_{1}}, \ldots, D_{\sigma_{l-1}}\right\}
$$

has $(t-1)$-normal crossings in $(W, E)$, and each of the divisors

$$
D_{\sigma_{l}}, \ldots, D_{\sigma_{m}}
$$

has only $t$-normal crossings in $(W, E)$ (note that $\mathcal{G}$ might be empty).

Since by assumption $D$ has $t$-normal crossings in $(W, E)$, note that:

(a) For every $j<l, G_{\sigma_{j}}=\emptyset$, and for every $j \geq l$, locally, at any closed point $x \in G_{\sigma_{j}}$, the divisor $D=\sum_{i=1}^{r} D_{i}$ can be identified with $D_{\sigma_{j}}$.

(b) If $E_{\sigma_{l}}=E \cup\left\{\cup D_{i}\right\}$, with $i \in\{1, \ldots, r\} \backslash \sigma_{l}$, then there is an open neighborhood $\mathcal{P}$ of $V\left(J_{\sigma_{l}}\right)$ such that $\left(W, E_{\sigma_{l}}\right)$ is a pair.

Now we apply Proposition 7.16 to $D_{\sigma_{l}},\left(\mathcal{P}, E_{\sigma_{l}}=E \cup\left\{\cup D_{i}\right\}\right)$, setting $s=t-1, E^{t-1}=\left\{\cup D_{i}\right\}$ with $i \in\{1, \ldots, r\} \backslash \sigma_{l}, E^{(t-1) \prime}=E$, and restricting the open sets, $V \cap \mathcal{P}$, and $V^{t-1} \cap \mathcal{P}$ (where $V^{t-1}$ is the subset of $W \backslash E$ where $D_{\sigma_{l}}$ has $(t-1)$-normal crossings). Thus, there exists a finite sequence of transformations of pairs,

$$
\left(\mathcal{P}_{0},\left(E_{\sigma_{l}}\right)_{0}\right)=\left(\mathcal{P}, E_{\sigma_{l}}\right) \leftarrow\left(\mathcal{P}_{1},\left(E_{\sigma_{l}}\right)_{1}\right) \leftarrow \ldots \leftarrow\left(\mathcal{P}_{N},\left(E_{\sigma_{l}}\right)_{N}\right)
$$

such that:

1. The divisor

$$
D_{\sigma_{l}}^{[N]}=\sum_{i \in \sigma_{l}} D_{i}^{[N]}
$$

has $(t-1)$-normal crossings in $\left(\mathcal{P}_{N},\left(E_{\sigma_{l}}\right)_{N}\right)$ (here we use the fact that the centers where we blow-up in sequence (7.17.1) have normal crossings with $\left\{\cup D_{i}\right\}_{i \notin \sigma_{l}}$, so for $i=1, \ldots, l-1$, each $D_{\sigma_{i}}^{[N]}$ has still $t-1$ normal crossings in $\left.\left(W_{N}, E_{N}\right)\right)$.

2. The morphism $\pi_{N}: \mathcal{P}_{N} \rightarrow \mathcal{P}$ induces an isomorphism on $V^{t-1} \cap U$, and hence on $V \cap U$.

Now observe that:

(A) Sequence (7.17.1) induces a sequence of transformations of pairs,

$$
\left(W_{0}, E_{0}\right)=(W, E) \leftarrow\left(W_{1}, E_{1}\right) \leftarrow \ldots \leftarrow\left(W_{N}, E_{N}\right) .
$$

(B) The divisors $\left\{D_{\sigma_{1}}^{[N]}, \ldots, D_{\sigma_{l}}^{[N]}\right\}$ have $(t-1)$-normal crossings in $\left(W_{N}, E_{N}\right)$.

(C) The morphism $W_{N} \rightarrow W$ induces an isomorphism on $V$. 
Iterating this argument, there is a finite sequence of transformations of pairs inducing an isomorphism on $V$,

$$
\left(W_{0}, E_{0}\right)=(W, E) \leftarrow\left(W_{1}, E_{1}\right) \leftarrow \ldots \leftarrow\left(W_{M}, E_{M}\right),
$$

such that for each $\sigma_{i} \in \Sigma$, all divisors $D_{\sigma_{i}}^{[M]}$ have $(t-1)$-normal crossings in $\left(W_{M}, E_{M}\right)$, and hence $D^{[M]}$ has $(t-1)$-normal crossings in $\left(W_{M}, E_{M}\right)$. Therefore, applying an inductive argument, there is a finite sequence of transformations of pairs,

$$
\left(W_{0}, E_{0}\right)=(W, E) \leftarrow\left(W_{1}, E_{1}\right) \leftarrow \ldots \leftarrow\left(W_{L}, E_{L}\right),
$$

such that $\pi_{L}: W_{L} \rightarrow W$ induces an isomorphism on $V$, and $D^{[L]}$ has 1-normal crossings in $\left(W_{L}, E_{L}\right)$. By Remark 7.15 (ii), this means that $\pi_{L}^{*}(D)=D^{[L]}+F_{L}$ has normal crossings, since $F_{L} \subset E_{L}$.

\section{On a class of regular schemes and on real and com- plex analytic spaces}

Up to now we have worked with schemes of finite type over fields. However, the constructions and proofs of Theorems 2.4 and 2.5 are valid for a much wider class of schemes (not necessarily of finite type over a field $\mathbf{k}$ ). Here we will focus on a class to which our techniques naturally extend.

Definition 8.1. We define $\mathcal{S}_{0}$ as the class of regular, equidimensional schemes $W$ containing a field, $\mathbf{k}$ of characteristic 0 (which may vary), satisfying the following two conditions:

(i) If $W$ is an $n$-dimensional $\mathbf{k}$-scheme in $\mathcal{S}_{0}$, then there is a finite affine open covering $\left\{\mathcal{U}_{i}\right\}_{i \in I}$, of $W$ such that for each $i \in I, \mathcal{U}_{i} \approx \operatorname{Spec}\left(R_{i}\right)$, for some Noetherian, regular k-algebra, $R_{i}$ with the additional property that $\operatorname{Der}_{\mathbf{k}}\left(R_{i}\right)$ is a finite projective $R_{i}$-module, locally of rank $n$.

(ii) If $\mathfrak{m}$ is a maximal ideal in $R_{i}$ then $\operatorname{dim}\left(R_{i}\right)_{\mathfrak{m}}=\mathrm{n}$ and $R_{i} / \mathfrak{m}$ is algebraic over $\mathbf{k}$.

Note that under condition (ii), $\mathbf{k}$ is a quasi-coefficient field at the localization at any closed point in the sense of [29, p. 274].

Remark 8.2. Note that:

(i) Any smooth scheme over a field of characteristic zero, as well as the spectrum of the completion, or henselization, of a local ring which is in $\mathcal{S}_{0}$ is in $\mathcal{S}_{0}$ (see [29, Theorem 90]).

(ii) The class of schemes in $\mathcal{S}_{0}$ is closed under monoidal transformations. This fact follows from [29, Appendix 40, Theorem 99, (3), (4)]. 
Theorem 8.3. The algorithms of desingularization and of principalization stated in Theorems 2.4 and 2.5 extend to the class of schemes in $\mathcal{S}_{0}$.

The reason why all the constructions that we have made for schemes of finite type over a field of characteristic zero extend to the class of schemes in $\mathcal{S}_{0}$, is that this class of schemes satisfies Property $\mathrm{D}$ stated below in Lemma 8.6: The algorithm of resolution is based in an inductive argument which in turn is based on the nice properties of the differential operator $\Delta$ defined on the class of smooth schemes over a field (see Sections 13 and 19). The class $\mathcal{S}_{0}$ parallels the class of compact (real or complex) analytic spaces with a well defined tangent bundle. It is easy to check that our results and developments extend to these class of spaces, too.

Definition 8.4. Let $R$ be a Noetherian, regular k-algebra, such that $\operatorname{Der}_{k}(R)$ is a finite projective $R$-module, locally of rank $n$, and assume that for each maximal ideal $\mathfrak{m}$ of $R, R_{j} / \mathfrak{m}$ is algebraic over $\mathbf{k}$. Then for each ideal $J$ of $R$ we define $\Delta(J)$ to be the ideal generated by

$$
\left\{\delta(f): \delta \in \operatorname{Der}_{\mathbf{k}}(R), f \in J\right\}
$$

(see [29, Appendix 40, Theorems 99, 102]).

Property $\mathrm{D}$ asserts that the differential operator $\Delta$ has the same nice properties when defined over the class of schemes in $\mathcal{S}_{0}$. This is enough to guarantee that the algorithm of resolution of basic objects, and hence the algorithms of principalization and resolution of singularities, hold within the class of schemes in $\mathcal{S}_{0}$. We make this idea more precise in the following paragraph.

8.5. The general strategy. We have approached the problems of desingularization and strong principalization of ideals in a unified way: by means of the notions of basic objects and resolution of basic objects. Given a basic object $B=(W,(J, b), E)$, where $W$ is smooth over a field $\mathbf{k}$ of characteristic zero, we have defined a function $\operatorname{ord}_{B}^{d}: \operatorname{Sing}(J, b) \rightarrow \mathbb{Q}(6.1)$, basically in terms of the function

$$
\text { ord }: W \rightarrow \mathbb{Z}
$$

given by the order of the ideal $J$ at points of $W$. In 13.7 we show that if $W$ is smooth over a field $k$, this function is upper-semi-continuous. Furthermore, because $W$ is smooth, we can define the $\Delta$ operator which acts on ideals of $W$. Note that the order of $J$ at the local regular ring $\mathcal{O}_{W, x}$ is, say, $d$, if and only if $x \in V\left(\Delta^{d-1}(J)\right) \backslash V\left(\Delta^{d}(J)\right)$. 
Resolution of basic objects (and hence desingularization and strong principalization) follow by induction on the dimension of the ambient space $W$. To be precise, and here is where a constraint on the characteristic of the underlying field $\mathbf{k}$ is imposed, the order of $J$ at $\mathcal{O}_{W, x}$ is $d$ if, and only if, the order of $\Delta^{d-1}(J)$ at $\mathcal{O}_{W, x}$ is 1 (see (P2) in 13.7 and Example 13.12). An element of order one in $\Delta^{d-1}(J) \subset \mathcal{O}_{W, x}$ defines a smooth hypersurface, say $H$ locally at $x \in W$ (so $\left.I(H) \subset \Delta^{d-1}(J)\right)$. After restriction, we may assume that $\operatorname{Sing}(J, b) \subset H$. Then we show that $H$ is a good candidate for induction on the ambient space, by showing that the inclusion $\left.I(H) \subset \Delta^{d-1}(J)\right)$ (and hence the inclusion $\operatorname{Sing}(J, d) \subset H$ ) is stable by transformations of basic objects. In doing so, we will only use the fact that $\Delta^{d-1}(J)$ is defined in terms of partial derivations (see Section 13).

Lemma 8.6. (Property D) If $W=\operatorname{Spec}(A)$ is a scheme in the class $\mathcal{S}_{0}$, $\mathfrak{p} \subset A$ is a prime ideal and $J$ is any ideal of $A$, then the order of $J A_{\mathfrak{p}}$ in the local regular ring $A_{\mathfrak{p}}$ is greater than or equal to $b$ if and only if $\Delta^{b-1}(J) \subset \mathfrak{p}$.

Proof: Let $R$ the localization of $A$ at a maximal ideal of $A$ containing $\mathfrak{p}$, and let $\hat{R}$ the completion of $R$. Then, the residue field of $\hat{R}$ is a finite extension $K$ of $\mathbf{k}$ and $\operatorname{Der}_{\mathbf{k}}(R)$ induces $\operatorname{Der}_{\mathbf{k}}(\hat{R})$ over $\hat{R}$ (see [29, Theorem 99, (4)]). By [29, Theorem 102], $R$ is excellent, $\operatorname{so} \operatorname{Spec}(\hat{R}) \rightarrow \operatorname{Spec}(R)$ is a faithfully flat morphism with regular fibers.

Note that the order of $J$ defines an upper-semi-continuous function on $\operatorname{Spec}(R)$. Since $J$ is finitely generated it suffices to check this when $J$ is principal. In that case, set $\bar{R}=R / J$; the order of $J$ at a prime $\mathfrak{p}$ is the multiplicity of the ring $\bar{R}$ at the prime ideal, say $\overline{\mathfrak{p}}$, induced by $\mathfrak{p}$, and multiplicity is upper-semi-continuous.

Assume first that $\mathfrak{p}$ is a regular prime ideal in $R$. Then it expands to a regular prime ideal in $\hat{R}$ and it is easy to see that the Property $\mathrm{D}$ holds for regular primes by checking that it holds at the completion, which is a ring of formal power series.

An arbitrary prime ideal $\mathfrak{p} \subset R$ is the intersection of all prime ideals of height $n-1$, where $n$ is the dimension of $R$. In fact, if $\operatorname{dim} R / \mathfrak{p}=n-h$ and $f \in R$ is not in $\mathfrak{p}$, one can find a prime ideal of height $n-1$ containing $\mathfrak{p}$ but not $f$ ( $\operatorname{set} \bar{f}_{1} \in R / \mathfrak{p}$ as the class of $f$, extend to $\bar{f}_{1}, \bar{f}_{2}, \ldots \bar{f}_{n-h} \in R / \mathfrak{p}$ a system of parameters, and now take $\mathfrak{q} \subset R$ by lifting a minimal prime ideal containing $\left.<\bar{f}_{2}, \ldots \bar{f}_{n-h}>\subset R / \mathfrak{p}\right)$.

Hence it suffices to check Property $D$ for $\mathfrak{p}$ a prime ideal defining a curve. Now we use a trick by Hironaka (see [23]), which consists in reducing to the regular case: Let

$$
\pi: W^{\prime} \rightarrow W
$$


be an an embedded desingularization of the curve defined by $\mathfrak{p}$, obtained as the composition of a finite sequence of quadratic transformations. Let $\mathfrak{p}_{\mathbf{l}}$ be the defining ideal of the desingularization of $\mathfrak{p}$, and set $\mathcal{O}_{W, x}$ and $\mathcal{O}_{W^{\prime}, y}$ as the local rings of $\mathfrak{p}$ and $\mathfrak{p}_{1}$ respectively. Then the lemma follows immediately from the fact that $\mathcal{O}_{W, x}$ and $\mathcal{O}_{W^{\prime}, y}$ are isomorphic, because in this case, we can identify the localizations of $\operatorname{Der}_{\mathbf{k}}\left(W_{1}\right)$ at $y$ with that of $\operatorname{Der}_{\mathbf{k}}(W)$ at $x$, and use the fact that the operator $\Delta$, is defined in terms of $\operatorname{Der}_{\mathbf{k}}(W)$ and that $\Delta$ is a well defined operator on the class of coherent ideals over $W$ (this can be checked at the completion at closed points).

\section{Non-embedded desingularization}

If $X \subset W$ is a closed subscheme then an embedded desingularization of $X$,

$$
\begin{array}{ccc}
W_{r} & \rightarrow & W \\
\cup & & \cup \\
X_{r} & & X
\end{array}
$$

defines a non-embedded desingularization, in the sense that it defines a proper birational morphism

$$
X_{r} \longrightarrow X_{0}
$$

such that $X_{r}$ is regular and the morphism is an isomorphism on $\operatorname{Reg}\left(X_{0}\right)$.

Our procedure of desingularization will also define a non-embedded desingularization of schemes which can be locally embedded in smooth schemes. This is not a restriction at all if we consider Noetherian separated schemes $X$ of finite type over a field $\mathbf{k}$. To prove that Theorem 2.4 extends to this class of schemes, we only have to prove that given two different embeddings of $X$ we obtain the same non-embedded desingularization.

Theorem 9.1. Let $X$ be a Noetherian separated scheme of finite type over a field of characteristic zero, $\mathbf{k}$. Consider two different closed immersions $X \subset W$ and $X \subset W^{\prime}$, where $W$ and $W^{\prime}$ are pure dimensional smooth schemes over $\mathbf{k}$. Let

$$
\begin{array}{ccccccc}
W & \leftarrow & W_{1} & \ldots & W_{r-1} & \leftarrow & W_{r} \\
\cup & & \cup & & \cup & & \cup \\
X & & X_{1} & \ldots & X_{r-1} & & X_{r}
\end{array}
$$

and

$$
\begin{array}{ccccccc}
W^{\prime} & \leftarrow & W_{1}^{\prime} & \ldots & W_{r-1}^{\prime} & \leftarrow & W_{r}^{\prime} \\
\cup & & \cup & & \cup & & \cup \\
X & & X_{1}^{\prime} & \ldots & X_{r-1}^{\prime} & & X_{r}^{\prime},
\end{array}
$$

be the embedded desingularizations provided by Theorem 2.4. 
Then sequences (9.1.1) and (9.1.2) define the same non-embedded desingularization of $X$, i.e., if

$$
\varphi: X_{r} \longrightarrow X, \quad \text { and } \quad \varphi^{\prime}: X_{r^{\prime}}^{\prime} \longrightarrow X_{0},
$$

are the two induced non-embedded desingularizations, then

$$
X_{r}=X_{r^{\prime}}^{\prime} \quad \text { and } \quad \varphi=\varphi^{\prime} .
$$

Moreover the number of blowing ups also coincide, i.e. $r=r^{\prime}$.

We refer to [19] for the proof of Theorem 9.1 (see also Remark 17.8), but let us mention here some auxiliary results which contribute to the proof of this theorem. These results are interesting by themselves, because they describe the behavior of the algorithm of resolution of singularities provided by Theorem 2.4 under regular extensions.

Proposition 9.2. Let $W$ be a pure dimensional smooth scheme over a field $\mathbf{k}$ and let $X \subset W$ be a closed subscheme. Then:

(i) If $W^{\prime} \longrightarrow W$ is an étale morphism and $X^{\prime} \subset W^{\prime}$ is the pull-back of $X$, then the desingularization of $X^{\prime} \subset W^{\prime}$ provided by Theorem 2.4 in 5.8, say

$$
\left(X_{r}^{\prime} \subset W_{r}^{\prime}\right) \longrightarrow\left(X^{\prime} \subset W^{\prime}\right)
$$

is the pull-back of the desingularization of $X \subset W$ provided by Theorem 2.4, say

$$
\left(X_{r} \subset W_{r}\right) \longrightarrow(X \subset W) .
$$

(ii) If $W^{\prime} \longrightarrow W$ is defined by an arbitrary extension of the base field, then the desingularization of the pull-back of $X$ in $W^{\prime}, X^{\prime} \subset W^{\prime}$ provided by Theorem 2.4,

$$
\left(X_{r}^{\prime} \subset W_{r}^{\prime}\right) \longrightarrow\left(X^{\prime} \subset W^{\prime}\right)
$$

is the pull-back of the desingularization of $X \subset W$ provided by Theorem 2.4,

$$
\left(X_{r} \subset W_{r}\right) \longrightarrow(X \subset W) .
$$

(iii) More generally, if $W^{\prime} \longrightarrow W$ is a regular map, and both $W$ and $W^{\prime}$ are schemes in $\mathcal{S}_{0}$, then the desingularization of the pull-back of $X$ in $W^{\prime}, X^{\prime} \subset W^{\prime}$ provided by Theorem 2.4,

$$
\left(X_{r}^{\prime} \subset W_{r}^{\prime}\right) \longrightarrow\left(X^{\prime} \subset W^{\prime}\right)
$$

is the pull-back of the desingularization of $X \subset W$ provided by Theorem 2.4,

$$
\left(X_{r} \subset W_{r}\right) \longrightarrow(X \subset W)
$$


Since the embedded desingularization stated in Theorem 2.4 is defined in terms of the resolution of a suitable defined basic object (see 5.8), Proposition 9.2 follows immediately from the next lemma:

Lemma 9.3. Let $(W,(J, b), E)$ be a basic object. Then the algorithm of resolution of basic objects defined in 5.5 and constructed in 14.8, satisfies the following additional property: If $W^{\prime} \longrightarrow W$ is a regular map, both $W$ and $W^{\prime}$ are in $\mathcal{S}_{0}$, and $J^{\prime} \subset \mathcal{O}_{W^{\prime}}$ and $E^{\prime}$ are respectively the pull-backs of $J$ and $E$ in $W^{\prime}$, then the resolution of $\left(W^{\prime},\left(J^{\prime}, b\right), E^{\prime}\right)$ defined in 5.5 is also the pull-back of the resolution of $(W,(J, b), E)$ defined in 5.5.

Proof: It is enough to observe that the proof of Theorem 14.8 relies on the functions ord and $n$ introduced in Definition 6.1, and that these functions are naturally compatible with regular maps between schemes in $\mathcal{S}_{0}$ :

Let $x^{\prime} \in W^{\prime}$, let $x=\pi\left(x^{\prime}\right)$, let $J \subset \mathcal{O}_{W}$ be a non-zero sheaf of ideals, and let $y \in W$ be a closed point contained in $\overline{\{x\}}$. Consider the diagram:

$$
\begin{gathered}
\mathcal{O}_{W, x} \\
\uparrow_{W, y}
\end{gathered} \mathcal{O}_{W, x^{\prime}}
$$

If $\operatorname{ord}_{x} J=b$, then $\Delta_{y}^{b-1}(J) \subset \mathcal{O}_{W, y}$ is a proper ideal. To check that

$$
\operatorname{ord}_{x} J=\operatorname{ord}_{x^{\prime}} J \mathcal{O}_{W^{\prime}}
$$

note that the diagram

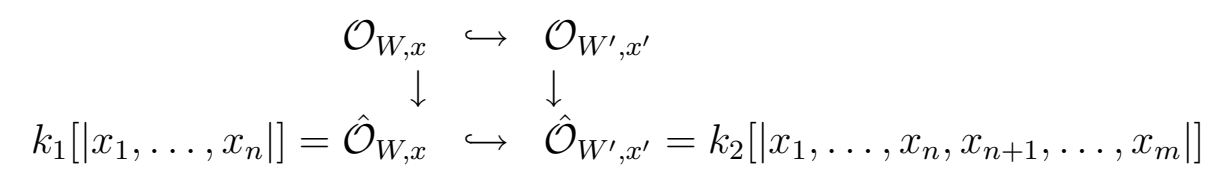

commutes, where $k_{1} \subset k_{2}$ since the characteristic is zero, and that all the maps are faithfully flat.

An important result in the study of equivariance for non-embedded schemes is the following lemma:

Lemma 9.4. Let $W$ and $W^{\prime}$ be pure dimensional schemes, smooth over $\mathbf{k}$, with $\operatorname{dim} W=\operatorname{dim} W^{\prime}$, and let $J \subset \mathcal{O}_{W}$ and $J_{0}^{\prime} \subset \mathcal{O}_{W^{\prime}}$, be two sheaves of ideals. Assume that for two points $\xi \in W$ and $\xi^{\prime} \in W^{\prime}$ there is an isomorphism $\Theta: \hat{\mathcal{O}}_{W, \xi} \longrightarrow \hat{\mathcal{O}}_{W^{\prime}, \xi^{\prime}}$ such that $\Theta(\hat{J})=\hat{J}^{\prime}$ (where $\hat{\mathcal{O}}_{W, \xi}$ and $\hat{\mathcal{O}}_{W^{\prime}, \xi^{\prime}}$ denote the completions of $\mathcal{O}_{W, \xi}$ and $\mathcal{O}_{W^{\prime}, \xi^{\prime}}$ respectively, and $\hat{J}_{0}=J_{0} \hat{\mathcal{O}}_{W_{0}, \xi_{0}}$, $\left.\hat{J}_{0}^{\prime}=J_{0}^{\prime} \hat{\mathcal{O}}_{W_{0}^{\prime}, \xi_{0}^{\prime}}\right)$. Then there is a common étale neighborhood $\tilde{\xi}_{0} \in \widetilde{W}_{0}$ of both $\xi_{0}$ and $\xi_{0}^{\prime}$, and and an ideal $\widetilde{J}_{0} \subset \mathcal{O}_{\widetilde{W}_{0}}$ such that

$$
\widetilde{J}_{0}=J_{0} \mathcal{O}_{\widetilde{W}_{0}}=J_{0}^{\prime} \mathcal{O}_{\widetilde{W}_{0}}
$$


Proof: If $\Theta$ arises from an isomorphism $\Theta: W_{0} \rightarrow W_{0}^{\prime}$ mapping $\xi_{0} \in W_{0}$ to $\xi_{0}^{\prime} \in W_{0}^{\prime}$, and $\Theta\left(J_{0}\right)=J_{0}^{\prime}$, then we it is enough to take $\widetilde{W}_{0}=W_{0}$ (note that an isomorphism is an étale map). We claim that this is the case in general, at least replacing $W$ and $W^{\prime}$ by suitable étale neighborhoods in the given points: Since the local rings $\mathcal{O}_{W, \xi} / J_{\xi}$ and $\mathcal{O}_{W^{\prime}, \xi^{\prime}} / J_{\xi^{\prime}}^{\prime}$ are formally isomorphic, then their henselizations are isomorphic (see $[5,2.6]$ ). Now, an isomorphism of these henselizations can be lifted to an isomorphism of the henselizations of the regular local rings, say $\Gamma: \overline{\mathcal{O}}_{W, \xi} \rightarrow \overline{\mathcal{O}}_{W^{\prime}, \xi^{\prime}}$, mapping $J \overline{\mathcal{O}}_{W, \xi}$ to $J^{\prime} \overline{\mathcal{O}}_{W^{\prime}, \xi^{\prime}}$. Since both henselizations are direct limits of étale neighborhoods, $\Gamma$ also defines an isomorphism, as indicated above, at suitable étale neighborhoods.

\section{Equiresolution. Families of schemes}

Once we fix a constructive algorithm of desingularization (i.e. once we attach to each variety a particular desingularization), it makes sense to ask whether one can classify varieties in accordance to their (algorithmic) desingularization. The formulation of this question already requires some clarification; but if we are to think of an embedded variety as a structure defined in terms of equations, it is conceivable to ask how the algorithm of desingularization will behave when the coefficients of these equations vary.

The questions about classification of varieties lead, quite naturally, to the notion of families of schemes. In this section we present briefly some results proved in [16] about the good behavior of the algorithm of desingularization in families and the notion of equiresolution.

Given a smooth morphism $W \rightarrow T$, we will denote by $W^{(t)}$, the fiber at the point $t \in T$, i.e.,

$$
W^{(t)}=W \times_{T} \operatorname{Spec}(k(t))
$$

where $k(t)$ is the residue field of $T$ at $t$. Note that $W^{(t)}$ is smooth over the field $k(t)$.

Definition 10.1. Given a smooth morphism $W \rightarrow T$, a family of embedded schemes is a closed subscheme $X \subset W$ such that induced morphism $X \rightarrow T$ is flat.

Example 10.2. Let $C \subset \mathbb{A}_{k}^{2}$ be a plane curve smooth over the field $k$ of characteristic zero. For an arbitrary scheme $T$ (say for instance a non reduced curve), $C \times T \subset \mathbb{A}_{k}^{2} \times T$ defines a flat family over $T$.

Example 10.3. Let $\mathbb{A}_{k}^{2} \rightarrow \mathbb{A}_{k}^{1}$ be the projection on first the coordinate. This is a smooth, and hence flat morphism. If we now take $W=\mathbb{A}_{k}^{2}=$ $\operatorname{Spec}\left(k\left[x_{1}, x_{2}\right]\right), T=\mathbb{A}_{k}^{1}$, and $X=V\left(x_{1} x_{2}\right) \subset W$, then this is not a family since $X \rightarrow T$ is not flat. 
Example 10.4. Let $\mathbb{A}_{k}^{4} \rightarrow \mathbb{A}_{k}^{1}$ be the projection on the first coordinate (i.e. defined by $k\left[x_{1}\right] \rightarrow k\left[x_{1}, x_{2}, x_{3}, x_{4}\right]$ the natural inclusion). Set $W=\mathbb{A}_{k}^{4}=$ $\operatorname{Spec}\left(k\left[x_{1}, x_{2}, x_{3}, x_{4}\right]\right), T=\mathbb{A}_{k}^{1}=\operatorname{Spec}\left(k\left[x_{1}\right]\right)$, and $X=V\left(x_{2}^{2}+x_{1} x_{3}^{3}, x_{4}\right) \subset W$. This example defines a family; the members of this family consist of irreducible singular curves, except at the the origin of $\mathbb{A}_{k}^{1}$ where the fiber is a double line.

So given a smooth morphism $W \rightarrow T$, we view $X \subset W$ as a structure defined, at least locally, by equations with coefficients in the scheme $T$. The requirement of flatness on $X \rightarrow T$ somehow avoids abrupt changes among the different fibers, a fact illustrated in Example 10.3.

There are various contexts where the notion of families of schemes appears, like for instance when dealing with Hilbert Schemes (see for instance [33]): Fix a projective space $\mathbb{P}_{k}^{n}$ and a Hilbert polynomial $F(t)$; then there is a scheme $S$, together with a setting $X \subset W=\mathbb{P}_{k}^{n} \times S \rightarrow S$ defining an embedded family such that the fibers parameterize all possible subschemes $Y \subset \mathbb{P}_{k}^{n}$ with Hilbert polynomial $F(t)$.

If we are to accept the notion of families as the right setting where to study the behavior of desingularization, a new problem arises, as illustrated in Example 10.4: It makes sense to formulate resolution of singularities for the general fibers, consisting of irreducible singular curves, but not for the particular fiber at the origin, consisting of a double line. Since we prove here that embedded desingularization is a byproduct of embedded principalization (see 5.8), we overcome this problem by simply replacing the use of embedded desingularization by principalization of the defining ideal. This will be explained with more detail in the next point of this section (see "Equisolvability I. Families parameterized by a smooth scheme $T$ " below). The results in desingularization will hold for a suitable class of families of schemes: The class of equisolvable families. Thus, our objectives are:

1. To introduce the notion of equisolvable families. The definition of equisolvability should be stable by fiber products, namely: If $X \subset$ $W \rightarrow T$, is equisolvable, and if $X_{1} \subset W_{1} \rightarrow T_{1}$, is defined by taking fiber products with $T_{1} \rightarrow T$, then the later should be equisolvable.

2. Given an arbitrary family $X \subset W \rightarrow T$, we will want to find a partition of algebraic nature on the underlying topological space of $T$, (a partition into locally closed sets $T_{\alpha}$ ) such that each restricted family, say $X_{\alpha} \subset W_{\alpha} \rightarrow T_{\alpha}$, is equisolvable.

Note that 2 would provide a formal setting to state that constructive resolution of singularities is algebraic on the coefficients, in fact we can take (2) as 
a definition, namely, that for any family $X \subset W \rightarrow T$, there is a partition, of algebraic nature, on the underlying topological space of $T$, such that each restricted family is equisolvable.

\section{Equisolvability I. Families parameterized by a smooth scheme $T$}

In this part we are going to restrict our attention to the case of families $X \subset W \rightarrow T$, where $T$ is smooth over a field of characteristic zero. Let $\mathcal{I}=\mathcal{I}(X) \subset \mathcal{O}_{W}$ be the defining ideal sheaf of $X$. We want to introduce conditions that insure that all the different members of the family of ideals

$$
\mathcal{G}=(\pi: W \rightarrow T, \mathcal{I})
$$

can be simultaneously principalized by using the algorithm. When this happens, we shall say that the family is equisolvable.

We propose two conditions (10.5 and 10.6), which turn out to be equivalent (see Theorem 10.7). Condition 10.5 does not explicitly involve the fibers of the family, but it requires, essentially, that the centers $C_{i}$ that appear in the principalization sequence that the algorithm associates to $\mathcal{I} \subset \mathcal{O}_{W}$, $(W, E)$ be "evenly spread" over the parameter space $T$. In condition 10.6, a "numerical" invariant is associated to the different points $t \in T$ (this invariant in defined in terms of the principalization sequences of the fibers); it is required that it be constant along $T$. Both approaches have their advantages, depending on the situation. One of our aims is to show that given an arbitrary family of ideals, it is possible to naturally stratify the parameter space $T$ as a union of locally closed sets so that, along each one, the restriction of the family is equisolvable.

Finally recall that the given embedded principalization algorithm induces an associated desingularization algorithm for couples $X \subset W$ (see 5.8). We shall say that a family of embedded schemes $X \subset W \rightarrow T$ is equisolvable (relative to the algorithm), if the associated family of ideals (10.4.1) is equisolvable. If the family of embedded schemes is such that all the fibers $X_{t}$ are reduced, then the desingularization sequence that the associated algorithm assigns to $X \subset W$ induces, on each fiber, the resolution sequence that corresponds to that fiber; that is, it has the property to be expected of a good notion of simultaneous resolution.

But this definition of equisolvability applies also to the case where some (or all the) fibers $X^{(t)}$ are non-reduced. So, we have a (we hope, reasonable) notion of equiresolution for families of embedded schemes where some fibers may be non-reduced. Since in many geometric problems it is unavoidable the presence of non-reduced fibers in families of schemes, it is an important feature. 
10.5. Condition of algorithmic equiresolution (AE). With the same notation as above, consider the basic object

$$
\left(W_{0},(\mathcal{I}, 1), E_{0}=\emptyset\right)
$$

and let

$$
\left(W_{0}, E_{0}\right) \longleftarrow\left(W_{1}, E_{1}\right) \longleftarrow \ldots \longleftarrow\left(W_{r}, E_{r}\right)
$$

be the embedded principalization sequence defined by the algorithm, where each $W_{i} \longleftarrow W_{i+1}$ is defined by blowing up at $C_{i}=\operatorname{Max}\left(f_{i}\right)$, which is determined by the functions defining the algorithm (see Theorem 2.5, Definition 5.5 and 5.9). Note that we get, by composition, smooth morphisms

$$
\pi_{i}: W_{i} \rightarrow T
$$

which induce morphisms $\rho_{i}: C_{i} \rightarrow T, i=0, \ldots, r-1$. We will say that the family of ideals $\mathcal{G}$ satisfies the condition of algorithmic equiresolution if the morphism

$$
\rho_{i}: C_{i} \rightarrow T
$$

is smooth, proper and surjective, for $i=0, \ldots, r$.

10.6. Condition $\tau$. Given a family of ideals $\mathcal{G}$ we are going to define a function $\tau_{\mathcal{G}}$ from the parameter space $T$ into a certain totally ordered set $\Lambda^{(d)}$ (which depends on $d=$ dimension of the fibers of $\pi: W \rightarrow T$ only):

(i) The values of $\tau_{\mathcal{G}}$ are sequences whose entries are either in $\mathbb{Z}$, or in the set $I^{(d)}$ introduced in Definition 5.5, or (possibly) $\infty$. This sequences will be ordered lexicographically. Therefore if we take $I_{d} \times \mathbb{Z}$ ordered lexicographically and set $L=I_{d} \times \mathbb{Z} \bigsqcup\{\infty\}$, we have that

$$
\Lambda^{(d)}=L \times L \times \ldots
$$

(ii) For each point $t \in T$, let $\left(W_{0}^{(t)},\left(\mathcal{I}^{(t)}, 1\right), E_{0}^{(t)}\right)$ be the fiber of $\left(W_{0},(\mathcal{I}, 1)\right.$, $\left.E_{0}=\emptyset\right)$ at $t \in T$, and now consider the sequence of transformations of pairs

$$
\left(W_{0}^{(t)}, E_{0}^{(t)}\right) \longleftarrow\left(W_{1}^{(t)}, E_{1}^{(t)}\right) \longleftarrow \ldots \longleftarrow\left(W_{r_{t}}^{(t)}, E_{r_{t}}^{(t)}\right)
$$

which provides an embedded principalization of the fiber of the ideal $\mathcal{I}$ over $t$. Let $c_{i}^{(t)}$ be the number of connected components of $C_{i}^{(\bar{t})}:=$ Max $f_{i}^{(\bar{t})}$. Here $C_{i}^{(\bar{t})}=C_{i} \times_{T} \overline{k(t)}$ is the geometric fiber $(\overline{k(t)}$ the algebraic closure of $k(t))$. Then we define

$$
\tau_{\mathcal{G}}(t)=\left(\max f_{0}^{(t)}, c_{0}^{(t)}, \max f_{1}^{(t)}, c_{1}^{(t)}, \ldots, \max f_{r_{t}}^{(t)}, c_{r_{t}}^{(t)}, \infty, \infty, \ldots\right) \in \Lambda^{(d)} .
$$

We will say that the family $\mathcal{G}$ satisfies the condition $\tau$ if $\tau_{\mathcal{G}}(t)$ is constant for all $t \in T$. 
Theorem 10.7. [16, Theorem 2.3] Let $T$ be an integral and smooth scheme, and let $\mathcal{G}=(\pi: W \rightarrow T, \mathcal{I})$ be a family of ideals. Assume that for all $i=0, \ldots, r-1$, the morphism $\rho_{i}: C_{i} \rightarrow T$ described in (10.5.3) is proper. Then $\mathcal{G}$ satisfies condition (AE) if and only if it satisfies condition $\tau$, and in either case, the principalization sequence (10.5.2) of $(W, \mathcal{I}, E)$ induces, by taking fibers, the principalization sequence of $\mathcal{G}(t):=\left(W^{(t)}, \mathcal{I}^{(t)}\right)$, for all $t \in T$.

The last part of the statement means the following. The length $r$ of the principalization sequence of $(W, \mathcal{I}, E)$ agrees with the length $r_{t}$ of the principalization sequence of any fiber $\mathcal{G}(t):=\left(W^{(t)}, \mathcal{I}^{(t)}\right)$; there is a natural identification of $\pi_{i}^{-1}(t)$ and $W_{i}^{(t)}(10.6 .1)$, for any $t \in T$, and via this identification, the restriction of $f_{i}$ to $\left(\pi_{i}^{-1}(t)\right)$ coincides with $f_{i}^{(t)}$, for $i=0, \ldots, r$.

Definition 10.8. [16, definition 1.10] A family of ideals $\mathcal{G}=(\pi: W \rightarrow T, \mathcal{I})$ is equisolvable if any of the equivalent conditions of Theorem 10.7 hold.

Note that the first hypothesis in Theorem 10.7 are automatically fulfilled if the morphism $\pi: W \rightarrow T$ is proper, in particular if it is projective.

Remark 10.9. Let $\mathcal{G}=(\pi: W \rightarrow T, \mathcal{I}, E)$ be a family of ideals. Let $T^{*} \rightarrow T$ a smooth morphism of smooth schemes, and set $\mathcal{G}^{*}=\left(\pi^{*}: W^{*} \rightarrow\right.$ $\left.T^{*}, \mathcal{I}^{*}, E^{*}\right)$ by taking fiber products. If $\mathcal{G}$ fulfills the conditions of the Theorem, then so does $\mathcal{G}^{*}$.

We mention now some results about families of embedded schemes.

Definition 10.10. We say that a family of embedded schemes $\mathcal{F}=(X \subset$ $W \rightarrow T)$ is equisolvable if the associated family of ideals is equisolvable.

Proposition 10.11. Assume that $\mathcal{F}=(X, W, \pi)$ is an equisolvable family of embedded schemes, where for all $t \in T$ the fiber $X^{(t)}$ is reduced. Then, for all $t \in T$, the resolution sequence (10.5.2) induces the resolution sequence of $\left(X^{(t)}, W^{(t)}\right)$.

Proof: By definition, the equisolvability of $\mathcal{F}$ means that the associated family of ideals $\mathcal{G}=(\pi: W \rightarrow T, \mathcal{I}(X), \emptyset)$ is equisolvable. Letting $W_{0}=W$, $\mathcal{I}=\mathcal{I}(X), E_{0}=\emptyset$, consider the principalization corresponding to $\mathcal{T}=$ $\left(W_{0}, \mathcal{I}, \emptyset\right)$. Equisolvability of $\mathcal{G}$ asserts that the principalization sequence of $\mathcal{G}$, induces that of the fiber $\mathcal{G}(t):=\left(W_{0}^{(t)}, \mathcal{I}_{0}^{(t)}, E_{0}^{(t)}\right)$, in the following sense. We have $C_{0}^{(t)}=C_{0} \cap W_{0}^{(t)}, f_{0}^{(t)}=f_{0} \mid W_{0}^{(t)}$ and the strict transform of $W_{0}^{(t)}$ in $W_{1}$ can be identified with $W_{1}^{(t)}$. Via this isomorphism, $f_{1}^{(t)}=$ $f_{1} \mid W_{1}^{(t)}, C_{1}^{(t)}=C_{1} \cap W_{1}^{(t)}$, and so on. Eventually, after $r$ steps, both the principalization sequence of $\mathcal{T}$ and $\mathcal{G}(t)$ simultaneously stop. 
Now $I(X) \mathcal{O}_{W_{0}^{(t)}}=I\left(X_{0}^{(t)}\right)$, and to obtain a desingularization sequence for $\left(X_{0}^{(t)}, W_{0}^{(t)}\right)$ we use the principalization sequence of the fiber $\mathcal{G}(t)=$ $\left(W_{0}^{(t)}, \mathcal{I}_{0}^{(t)}, \emptyset\right)$. It is clear that, via the identification of $W_{i}^{(t)}$ with a suitable subscheme of $W_{i}$, for all possible index $i, X_{i}^{(t)}$ corresponds to the strict transform of $X_{0}^{(t)}$ to $W_{i}$. Moreover, the length $s_{t}$ of the resolution sequence of $\left(X_{0}^{(t)}, W_{0}^{(t)}\right)$ is constant, equal to the length $s$ of the resolution sequence of $(X, W)$. In fact $s$ (resp. $s_{t}$ ) is the unique index such that the proper transform $X_{s} \subset W_{s}$ (resp. $\left.X_{s}^{(t)} \subset W_{s}^{(t)}\right)$ has the same codimension as the center $C_{s}$ (resp. $\left.C_{s}^{(t)}\right)$ (see 5.8). But $\operatorname{codim}\left(X_{i}^{(t)}, W_{i}^{(t)}\right)=\operatorname{codim}\left(X_{i}, W_{i}\right)$ and, since $\mathcal{G}$ is equisolvable, $\operatorname{codim}\left(C_{i}^{(t)}, W_{i}^{(t)}\right)=\operatorname{codim}\left(C_{i}, W_{i}\right)$. This proves our contention about the indices. Since the desingularization functions are defined by restriction, the proposition is proved.

\section{Equisolvability II. Stratification of Hilbert schemes}

Definition 10.12. A general family of embedded schemes is a pair

$$
\mathcal{F}=(j: X \rightarrow W, \pi: W \rightarrow T)
$$

where $\pi: W \rightarrow T$ is a smooth morphism of equidimensional Noetherian schemes over a field $\mathbf{k}$ of characteristic zero, $j$ is a closed immersion and $p:=\pi \circ j: X \rightarrow T$, is flat (but we do not assume that $W$ or $T$ are regular).

Definition 10.13. A general family of ideals is a pair

$$
\mathcal{G}=(\pi: W \rightarrow T, \mathcal{I})
$$

where $\pi: W \rightarrow T$ is a smooth morphism (but we do not require $W$ or $T$ to be regular or irreducible). All fibers $\mathcal{G}(t):=\left(W^{(t)}, \mathcal{I}^{(t)}\right)$ give ideals $\mathcal{I}^{(t)} \subset \mathcal{O}_{W^{(t)}}$.

Remark 10.14. Note that the function $\tau_{\mathcal{G}}: T \rightarrow \Lambda^{(d)}$ introduced in 10.6 still can be defined for any general family. If $T$ admits a desingularization $T_{1} \rightarrow T$ then $\mathcal{G}_{1}$ (obtained from $\mathcal{G}$ by base change to $T_{1}$ ) is a family in the sense of Definition 10.4.1. Hence Theorem 10.7 applies to $\mathcal{G}_{1}$. Since $\tau_{\mathcal{G}}$ is defined in terms of the fibers only, from the properness of $T_{1} \rightarrow T$ we see that the conclusion of Theorem 10.7 is also valid for the family $\mathcal{G}$.

Given a general family of embedded schemes, $\mathcal{F}=(j: X \rightarrow W, \pi: W \rightarrow$ $T$ ), we can associate to it a general family of ideals (as we did in (10.4.1)), say $\mathcal{G}=(\pi: W \rightarrow T, \mathcal{I})$ (Definition 10.13), where $\mathcal{I}=I(X)$. For simplicity, in the sequel we denote by $\tau_{\mathcal{F}}$ the function $\tau_{\mathcal{G}}$ corresponding to the family of ideals $\mathcal{G}$. 
10.15. Now fix the following objects:

(i) A graded algebra $S$ over a field $k$ of characteristic zero, finitely generated by elements of degree one, such that $W=\operatorname{Proj}(S)$ is smooth of finite type over $k$, of dimension $n$ (which belongs to the class $\mathcal{S}_{0}$ ).

(ii) An element $\alpha \in \Lambda^{(d)}$.

(iii) A polynomial $Q$ with rational coefficients.

Let $\mathcal{H}(W, \alpha, Q)$ be the class of all general families of embedded and reduced schemes $\mathcal{F}_{T}$, of the form $\left(j: X_{T} \rightarrow W_{T}, \pi: W_{T} \rightarrow T\right)$ where $\pi$ is obtained from $W \rightarrow \operatorname{Spec}(k)$ by base change, and $X_{T} \subset W_{T}$ is a closed subscheme such that the induced projection $X_{T} \rightarrow T$ is flat ( $j$ being the inclusion). We require also for $\mathcal{F}_{T} \in \mathcal{H}(W, \alpha, Q)$ that:

(0) $T$ is a reduced scheme of finite type over $k$.

(1) For all $t \in T, \tau_{\mathcal{F}, T}(t)=\alpha$ (hence the pull-back of this general family via $T^{\prime} \rightarrow T$, with $T^{\prime}$ regular is equisolvable since it satisfies condition $\tau$ ).

(2) If $\left(X^{(t)}, W\right)$ is the couple induced by $\left(X_{T}, W_{T}\right)$ over $t \in T$, then $Q$ is the Hilbert polynomial (relative to the line bundle corresponding to $S(-1)$ ) of the embedded scheme $X^{(t)} \subset W$.

Now we can state the following theorem:

Theorem 10.16. [16, 4.10] Under the conditions stated in 10.15 (and letting $H:=\mathcal{H}(W, \alpha, Q))$ there is a universal object in the class of general families in $H$ (which will be called a universal $(\alpha, Q)$-equisolvable family). That is, there is a general family, $\mathcal{F}_{H(\alpha, Q)}$ defined by $\left(X_{H(\alpha, Q)} \subset W_{H(\alpha, Q)}\right)$, $\left.W_{H(\alpha, Q)} \rightarrow H(\alpha, Q)\right)$ such that for each general family $\mathcal{F}_{T}$ in $H$, there is a unique morphism $T \rightarrow H(\alpha, Q)$ so that $\mathcal{F}_{T}$ is the pull-back of $\mathcal{F}_{H(\alpha, Q)}$.

Proof: Consider the Hilbert scheme $H(Q)$, parameterizing subschemes of the projective variety $W$ having Hilbert polynomial $Q$. We refer here to $[31$, p. 21 (c)] for a summary of results on Hilbert schemes. Let $X(Q) \subset$ $W \times H(Q)$ be the universal family; note that $(X(Q), W \times H(Q))$ together with the projection $W \times H(Q) \rightarrow H(Q)$ defines a general family, say $\mathcal{F}(W, Q)$. Then [16, Theorem 4.8] says that $\tau_{\mathcal{F}(W, Q)}: H(Q) \rightarrow \Lambda^{(n)}$ is an LC-function. Hence its fibers define a partition of $H(Q)$ into a disjoint union of locally closed subsets. If $\alpha \in \Lambda^{(n)}$, let $H(\alpha, Q):=\left[\tau_{\mathcal{F}(W, Q)}\right]^{-1}(\alpha)$. Given a general family, say $\mathcal{F}_{T}$, in $H$, since the natural morphism $T \rightarrow H(Q)$ obtained by universality of Hilbert schemes has constant value $\alpha$, it becomes clear that it factors though $H(\alpha, Q)$ and vice versa, proving the theorem. 
Definition 10.17. An $L C$-partition of a scheme $T$, is an expression of $T$ as a disjoint union of locally closed subsets (or subschemes, with the reduced structure).

Remark 10.18. In Theorem 10.16 we have introduced an LC-partition in the Hilbert scheme $H(Q)$ which is naturally related to equiresolution, by means of the (reduced) subschemes $H(\alpha, Q)$. We might call this a stratification of $H(Q)$ but, in the literature, this term is often used in a more restricted sense. For instance, in [27] the notion of stratification of $T$ is introduced as an LC-partition (each set thereof is called a stratum) with the following properties:

(i) The boundary of each stratum is a union of strata.

(ii) The singular locus of the closure of each stratum is a union of stratum.

(iii) Each stratum is smooth.

In $[27,2.5$ (c) $]$ it is remarked that given an LC-partition of an algebraic variety $\mathrm{T}$, one can attach to this partition a coarsest stratification of $T$, with the property each locally closed subset of the partition is a union of strata. Here coarsest means that any other stratification with this property is a refinement of the first. So, if we want to obtain a stratification of the Hilbert scheme $H(Q)$, in the sense of [27], and naturally related to equiresolution, all what we need it to take the coarsest stratification associated to the LCpartition described in Theorem 10.16.

\section{Bodnár-Schicho's computer implementation}

In [35, Theorem 7.3.] and [18, Theorem 7.13.] give an algorithm of resolution of basic objects. Based on this construction, G. Bodnár and J. Schicho wrote a computer program which resolves basic objects: Given a basic object, $(W,(J, b), E)$ the program provides the resolution defined in terms of functions $f_{i}^{d}$, as indicated in Definition 5.5, (c) (see [10] and [11]). Their program is available at

http://www.risc.uni-linz.ac.at/projects/basic/adjoints/blowup

As a consequence, the authors provided a computer program which computes the resolution of singularities of a given hypersurface (cf. [10] and [11]). The main obstacle to generalize this process to varieties of arbitrary codimension was that the classical approach for desingularization was based on reducing to the hypersurface case, and in this process, algorithms of division and strict transforms of ideals are involved.

In the new approach of desingularization presented in this paper all this is avoided. The short proofs of embedded resolution of singularities and 
embedded principalization of ideals presented in 5.8 and 5.9 are only based on the existence of an algorithm of resolution of basic objects. As a consequence, based on these ideas, G. Bodnár and J. Schicho have been able to adapt their program to produce one that resolves the singularities of varieties of arbitrary codimension. Also, the program can compute embedded principalization of ideals. We refer to Section 18 for some hints on how their computer program works.

About the performance of the program, the complexity of computations seems to be very high but, by now, we do not know any bound for complexity. In practice "easy" examples for $\operatorname{dim} W \leq 4$ may be computed. For instance if the input is an ideal $J \subset \mathbb{Q}\left[X_{1}, \ldots, X_{n}\right]$ then the resolution of the variety defined by $J$ can be computed if $n \leq 4$ and the degree of the generators of $J$ is not bigger than 7 or 8 . The first versions of the program were implemented in Maple, but the most recent version use Singular, which performs much better all standard basis computations. For example, the resolution of the Whitney umbrella takes 1.230 seconds of CPU time in a PowerPC G3 at $700 \mathrm{Mhz}$. But sometimes one could find an example which seems "easy" but it requires hard middle computations, since coefficients ideals are complicated. The most recent version has the option to compute in some localized rings and improves the performance in some cases. This option is possible using the features of Singular for computing some localization of polynomial rings, see [9].

\section{Part IV. Preliminaries for constructive reso- lutions}

We will prove resolution of basic objects by induction on the dimension of the ambient space: To find a resolution of a $d$-dimensional basic object $(W,(J, b), E)$ we will associate to it, at least locally at each point of $W$, a $(d-1)$-dimensional basic object $(Z,(\mathcal{A}, e), G)$ in such a way that a resolution of $(Z,(\mathcal{A}, e), G)$, is equivalent to a resolution of $(W,(J, b), E)$ in some sense that we will make precise in the forthcoming sections. This will force us to introduce some machinery:

1. The notions of equivalence, inclusion and intersection of basic objects (see Section 12).

2. The notion and properties of the $\Delta$ operator, which will provide us the way to find $(Z,(\mathcal{A}, e), G)$ given $(W,(J, b), E)$ (see Definition 13.10).

3. The notion of general basic object: Given a $d$-dimensional basic object $(W,(J, b), E)$, in some situations we will associate to it locally, at each point of $W$, a $(d-1)$-dimensional basic object. In other words, we will 
associate to $(W,(J, b), E)$, a collection of $(d-1)$-dimensional basic objects, $\left(\widetilde{W}_{\alpha},\left(I_{\alpha}, a_{\alpha}\right), E_{\alpha}\right)$. This family of $(d-1)$-dimensional basic objects will be what we call a general basic object (see Section 14).

\section{Intersections, inclusions and equivalence of basic objects}

12.1. There are two types of information encoded in the notion of basic object: A pair $(W, E)$, and a closed subset $F=\operatorname{Sing}(J, b)$. Moreover, any sequence of transformation of basic objects

$(12.1 .1)$

$(W,(J, b), E)=\left(W_{0},\left(J_{0}, b\right), E_{0}\right) \leftarrow\left(W_{1},\left(J_{1}, b\right), E_{1}\right) \leftarrow \ldots \leftarrow\left(W_{k},\left(J_{k}, b\right), E_{k}\right)$,

induces a sequence of transformations of pairs with the same centers,

$$
\left(W_{0}, E_{0}\right) \longleftarrow\left(W_{1}, E_{1}\right) \longleftarrow \cdots \longleftarrow\left(W_{k}, E_{k}\right)
$$

together with a collection of closed sets $F_{i}=\operatorname{Sing}\left(J_{i}, b\right)$ included in $W_{i}$, for $i=0,1, \ldots, k$.

12.2. Inclusions of basic objects. We say that $(W,(L, b), E)$ is contained in $(W,(J, b), E)$,

$$
(W,(L, s), E) \subset(W,(J, b), E)
$$

if $\operatorname{Sing}(L, s) \subset \operatorname{Sing}(J, b)$, and

(i) For any sequence of transformations of the basic object $(W,(L, s), E)$,

$$
(W,(L, s), E) \longleftarrow\left(W_{1},\left(L_{1}, s\right), E_{1}\right) \longleftarrow \ldots \longleftarrow\left(W_{k},\left(L_{k}, s\right), E_{k}\right),
$$

the corresponding induced sequence of transformations of pairs as in (12.1.2) defines a permissible sequence

$$
(W,(J, b), E) \longleftarrow\left(W_{1},\left(J_{1}, b\right), E_{1}\right) \longleftarrow \ldots \longleftarrow\left(W_{k},\left(J_{k}, b\right), E_{k}\right),
$$

with the additional property that for $i=1, \ldots, k$,

$$
\operatorname{Sing}\left(L_{i}, s\right) \subset \operatorname{Sing}\left(J_{i}, s\right) .
$$

(ii) If $U \subset W$ is an open set then we require the previous property to hold for the restrictions $(W,(L, s), E)_{U}$ and $(W,(J, b), E)_{U}$ for any $U$ (see $5.6(2))$.

Example 12.3. Fix a pair $(W, E)$ and two sheaves of ideals $J \subset J^{\prime} \subset \mathcal{O}_{W}$. Then clearly

$$
\left(W,\left(J^{\prime}, b\right), E\right) \subset(W,(J, b), E) .
$$


Remark 12.4. We have just introduced a notion of inclusion of basic objects. It may also happen that there is a double inclusion, namely that $(W,(L, s), E)$ is contained in $(W,(J, b), E)$ and $(W,(J, b), E)$ is contained in $(W,(L, s), E)$ (i.e. $i d:(W, E) \rightarrow(W, E)$ defines an isomorphism of both basic objects as in Definition 4.6). Then we will say that they are equivalent, and will use the notation

$$
(W,(J, b), E) \cong(W,(L, s), E)
$$

Remark 12.5. Note that we view a basic object as a pair, and a way of defining transformations of pairs and closed sets: Two equivalent basic objects $(W,(J, b), E) \cong(W,(L, s), E)$ define exactly the same transformations of pairs and the same closed sets. The invariants involved in the constructive resolution of these basic objects (the functions $f_{i}$ introduced in Definition 5.5), will be defined in terms of the closed subsets described by the basic objects. So the invariants attached to $(W,(J, b), E)$ will coincide with those attached to $(W,(L, s), E)$. In particular the constructive resolution of both basic objects given by Definition 5.5 will be the same (i.e. will be defined by the same sequence of transformations).

Example 12.6. If we set $(L, s)=\left(J^{2}, 2 b\right)$, then

$$
(W,(J, b), E) \cong(W,(L, s), E)
$$

(see also Example 4.8).

12.7. Intersections of basic objects. Given two basic objects,

$$
(W,(J, b), E) \quad \text { and } \quad(W,(I, c), E)
$$

we define its intersection, $(W,(J, b), E) \cap(W,(I, c), E)$, as the basic object $(W,(K, e), E)$ where $K=J^{c}+I^{b}$ and $e=b c$.

The intersection $(W,(K, e), E)$ is contained in both $(W,(J, b), E)$ and $(W,(I, c), E)$, in the sense defined in 12.2 . So any transform $(W,(K, e), E) \longleftarrow$ $\left(W_{1},\left(K_{1}, e\right), E_{1}\right)$ induces two transformations

$$
(W,(J, b), E) \longleftarrow\left(W_{1},\left(J_{1}, b\right), E_{1}\right) \text { and }(W,(I, c), E) \longleftarrow\left(W_{1},\left(I_{1}, c\right), E_{1}\right),
$$

and it can be checked that

$$
\left(W_{1},\left(K_{1}, e\right), E_{1}\right)=\left(W_{1},\left(J_{1}, b\right), E_{1}\right) \cap\left(W_{1},\left(I_{1}, c\right), E_{1}\right)
$$

so that the definition of intersection is compatible with transformations. 
Example 12.8. Let $\left(W_{0},\left(J_{0}, b\right), E_{0}\right)$ be a basic object, let $H_{0} \subset W_{0}$ be a regular hypersurface and consider the basic object $\left(W_{0},\left(\mathcal{I}\left(H_{0}\right), 1\right), E_{0}\right)$. Then,

Note that a resolution of $\left(W_{0},\left(K_{0}, b\right), E_{0}\right)$,

$$
\begin{aligned}
\left(W_{0},\left(J_{0}, b\right), E_{0}\right) \cap\left(W_{0},\left(\mathcal{I}\left(H_{0}\right), 1\right), E_{0}\right) & =\left(W_{0},\left(J_{0}+\mathcal{I}\left(H_{0}\right)^{b}, b\right), E_{0}\right) \\
& =\left(W_{0},\left(K_{0}, b\right), E_{0}\right) .
\end{aligned}
$$

$$
\left(W_{0},\left(K_{0}, b\right), E_{0}\right) \leftarrow \ldots \leftarrow\left(W_{n-1},\left(K_{n-1}, b\right), E_{n-1}\right) \leftarrow\left(W_{n},\left(K_{n}, b\right), E_{n}\right)
$$

induces a sequence of transformations of basic objects for both $\left(W_{0},\left(J_{0}, b\right), E_{0}\right)$

$$
\left(W_{0},\left(J_{0}, b\right), E_{0}\right) \leftarrow \ldots \leftarrow\left(W_{n-1},\left(J_{n-1}, b\right), E_{n-1}\right) \leftarrow\left(W_{n},\left(J_{n}, b\right), E_{n}\right)
$$

and $\left(W_{0},\left(\mathcal{I}\left(H_{0}\right), b\right), E_{0}\right)$,

$$
\left(W_{0},\left(\mathcal{I}\left(H_{0}\right), b\right), E_{0}\right) \leftarrow \ldots \leftarrow\left(W_{n-1},\left(\mathcal{I}\left(H_{0}\right)_{n-1}, b\right), E_{n-1}\right) \leftarrow\left(W_{n},\left(\mathcal{I}\left(H_{0}\right)_{n}, b\right), E_{n}\right) .
$$

Since $H_{0}$ is a regular hypersurface, $\mathcal{I}\left(H_{0}\right)_{n}$ is the sheaf of ideals defining the strict transform of $H_{0}$, say $H_{0, n} \subset W_{n}$ (see Remark 3.9 (2)), and since $(12.8 .1)$ is a resolution of $\left(W_{0},\left(K_{0}, b\right), E_{0}\right)$, then

$$
\operatorname{Sing}\left(J_{n}, b\right) \cap H_{0, n}=\emptyset \text {. }
$$

12.9. Basic objects defined over different pairs. We have defined operations of inclusions and intersections of two basic objects over a same pair. Now let $\left(W, E=\left\{H_{1}, . ., H_{r}\right\}\right)$ be a pair and let $Z \subset W$ be a closed smooth subscheme. Assume that each $H_{i}$ intersects $Z$ transversally at $\widetilde{H}_{i}$ for $i=1, \ldots, r$, and that $\left(Z, \widetilde{E}=\left\{\widetilde{H}_{1}, \ldots, \widetilde{H_{r}}\right\}\right)$ is a pair (for instance, this will be the case if $E=\widetilde{E}=\emptyset)$.

Note that a closed subset in $Z$ is a closed subset in $W$ and that any sequence of transformation of the pair $(Z, \widetilde{E})$ induces one over $(W, E)$ (see Definition 2.1). Note also that

$$
(Z,(A, e), \widetilde{E}) \subset(W,(\mathcal{I}(Z), 1), E),
$$

since any sequence of transformations over the first induces one over the second, with inclusion of the corresponding closed sets. In this context we can also have two basic objects with a double inclusion as in Remark 12.4 (an inclusion of the corresponding closed sets), and then we will say they are equivalent, and denote it by

$$
(Z,(A, e), \widetilde{E}) \cong(W,(J, b), E) .
$$

If $\operatorname{dim}(Z)=d^{\prime}$, we will abuse notation by saying that $(W,(J, b), E)$ has a structure of a $d^{\prime}$-dimensional basic object.

Example 12.10. Let $W$ be smooth of dimension $d$, and let $X \subset W$ be a curve which is defined by $d-1$ global sections $f_{1}, \ldots, f_{d-1}$ in $\mathcal{O}_{W}$. Assume that $Z=V\left(<f_{1}>\right)$ is smooth. Then $(W,(J, 1), \emptyset) \cong(Z,(A, 1), \emptyset)$ where $J=<f_{1}, \ldots, f_{d-1}>\subset \mathcal{O}_{W}$ and $A=J \mathcal{O}_{Z}$. 


\section{The differential operator and the inductive nature of the algorithm}

The objective of this section is to state Proposition 13.3, which is a key step in the inductive proof of Theorem 3.10. Proposition 13.3 says that we can associate (at least locally) a $d-1$ dimensional basic object to some class of $d$-dimensional basic objects (we will refer to them as basic objects within the case $\operatorname{ord}_{\mathcal{B}} \cong 1$ - see Definition 13.2). The rest of this section is devoted to giving a flavor of the idea behind of Proposition 13.3 as well as to explain the role of the sheaf of differentials in this process. The proof of Proposition 13.3 will be postponed to Section 19. Note that all arguments exposed here extend to the class of schemes described in Definition 8.1.

Definition 13.1. Given a sheaf of ideals $J \subset \mathcal{O}_{W}$ we will denote by $R(1)(F)$ the closed subset of points where $F=V(J)$ has codimension 1 in $W$.

Definition 13.2. Let $\mathcal{B}=(W,(J, b), E)$ be a basic object, and consider the equivariant function introduced in Section 6 ,

$$
\begin{aligned}
\operatorname{ord}_{\mathcal{B}}: F=\operatorname{Sing}(J, b) & \rightarrow \mathbb{Q} \\
x & \rightarrow \frac{\nu_{J}(x)}{b} .
\end{aligned}
$$

We will say that $\mathcal{B}$ is within the case $\operatorname{ord}_{\mathcal{B}} \cong 1$ if the maximum value of $\operatorname{ord}_{\mathcal{B}}: F \rightarrow \mathbb{Q}$ is 1 .

Proposition 13.3. Let $\mathcal{B}=(W,(J, b), E)$ be a d-dimensional basic object, assume that $E=\emptyset$ and that $\operatorname{ord}_{B} \cong 1$. Then:

a) There is an open covering $\left\{U_{\alpha}\right\}_{\alpha \in \Lambda}$, and for each index $\alpha \in \Lambda$ a closed and smooth hypersurface $\widetilde{W}_{\alpha} \subset U_{\alpha}$, such that if $\left(U_{\alpha},\left(J_{\alpha}, b\right), \emptyset\right)$ is the restriction of $(W,(J, b), \emptyset)$ to $U_{\alpha}$ as in Remark 4.5, then

$$
\left(U_{\alpha},\left(J_{\alpha}, b\right), \emptyset\right) \subset\left(U_{\alpha},\left(\mathcal{I}\left(\widetilde{W}_{\alpha}\right), 1\right), \emptyset\right) .
$$

b) If $U_{\alpha} \cap R(1)(\operatorname{Sing}(J, b))=\emptyset$ then $\left(U_{\alpha},\left(J_{\alpha}, b\right), \emptyset\right)$ has structure of $(d-1)$ dimensional basic object, i.e. there is a $(d-1)$-dimensional basic object $\left(\widetilde{W}_{\alpha},\left(\mathcal{A}_{\alpha}, e_{\alpha}\right), \emptyset\right)$ such that

$$
\left(U_{\alpha},\left(J_{\alpha}, b\right), \emptyset\right) \cong\left(\widetilde{W}_{\alpha},\left(\mathcal{A}_{\alpha}, e_{\alpha}\right), \emptyset\right) .
$$

Remark 13.4. This proposition is the key point of our inductive argument. We are using the notation of 12.9 (here $E=\emptyset$ ). In general, when $E \neq \emptyset$, our constructive resolution will not follow only from induction on the dimension of ambient space $W$, but rather from induction on pairs $(W, E)$. The function $n(x)$ introduced in 6.2 will allow us to treat the case $(W, E)$ when $E \neq \emptyset$. 
Definition 13.5. Let $W$ be a smooth scheme over a field $\mathbf{k}$ and let $\Omega_{W / \mathbf{k}}$ be the (locally free) sheaf of differentials of $W$ over k. Given a closed point $\xi \in W$ and a regular system of parameters $\left\{x_{1}, \ldots, x_{n}\right\} \subset \mathcal{O}_{W, \xi}$, let

$$
\left\{\frac{\partial}{\partial x_{1}}, \ldots, \frac{\partial}{\partial x_{n}}\right\}
$$

be a basis of the dual of the $\mathcal{O}_{W, \xi}$-module, $\left(\Omega_{W / \mathbf{k}}^{1}\right)_{\xi}$. We will express the differential operator $\Delta$ in terms of this basis although it will be independent of any choice of parameters.

Definition 13.6. (See also Section 8). Let $W$ be a smooth scheme over a field $\mathbf{k}$, and let $J \subset \mathcal{O}_{W}$ be a non-zero sheaf of ideals. Given a closed point $\xi \in W$ and a regular system of parameters $\left\{x_{1}, \ldots, x_{n}\right\} \subset \mathcal{O}_{W, \xi}$, we define $\Delta(J) \subset \mathcal{O}_{W}$ as the ideal locally generated by

$$
\left\{f_{i}\right\}_{i=1}^{r} \cup\left\{\frac{\partial f_{i}}{\partial x_{j}}\right\}_{\substack{i=1, \ldots, r \\ j=1, \ldots, n}}
$$

where $J_{\xi}=\left\langle f_{1}, \ldots, f_{r}\right\rangle$. We also define by induction $\Delta^{i}(J)=\Delta\left(\Delta^{i-1}(J)\right)$, for $i \geq 1$, where $\Delta^{0}(J)=J$ and $\Delta^{1}(J)=\Delta(J)$. Note that $\Delta(J)$ is independent of the choice of parameters.

13.7. Properties of the $\Delta$ operator. If $J$ is a non-zero sheaf of ideals at any irreducible component of the smooth scheme $W$ of characteristic zero, then the ideal $\Delta(J)$ satisfies the following properties:

P1. $J \subset \Delta(J) \subset \cdots \subset \Delta^{b}(J)=\mathcal{O}_{W}$ for some $b \in \mathbb{N}$.

P2. For any point $\xi \in W, \nu_{\mathcal{O}_{W, \xi}}\left(J_{\xi}\right)=b>1$ if and only if

$$
\nu_{\mathcal{O}_{W, \xi}}\left(\Delta(J)_{\xi}\right)=b-1 \text {. }
$$

In particular $\nu_{\mathcal{O}_{W, \xi}}(J)=b>0$ if and only if

$$
\nu_{\mathcal{O}_{W, \xi}}\left(\Delta^{b-1}(J)\right)=1
$$

P3. For any point $\xi \in W, \nu_{\mathcal{O}_{W, \xi}}(J) \geq b>1$ if and only if $\xi \in V\left(\Delta^{b-1}(J)\right)$.

13.8. Basic objects and the $\Delta$ operator. Let $(W,(J, b), E)$ be a basic object over a field $\mathbf{k}$ of characteristic zero, then by looking at the Taylor expansion of the generators of $J$ we have that

$$
V\left(\Delta^{b-1}(J)\right)=\operatorname{Sing}(J, b) .
$$

See also 8.6. 
Remark 13.9. Given a basic object $\mathcal{B}=(W,(J, b), E)$, set $F=\operatorname{Sing}(J, b)$, and note that the following statements are equivalent:

(a) The maximum value of $\operatorname{ord}_{\mathcal{B}}: F \rightarrow \mathbb{Q}$ is 1 (i.e. $B$ is within the case $\left.\operatorname{ord}_{\mathcal{B}} \cong 1\right)$.

(b) For every $x \in F, \operatorname{ord}_{\mathcal{B}}(x)=1$.

(c) The order of $J_{x}$ at $\mathcal{O}_{W, x}$ is $b$ for every $x \in F$.

(d) The order of $\Delta^{b-1}(J)$ is zero or one at every point of $W$ and the order is one at points of $F$.

Definition 13.10. Let $(W,(J, b), E)$ be a basic object, and let $Z \subset W$ be a closed and smooth subscheme such that

1. For any $H \in E$, the closed subschemes $Z$ and $H$ intersect transversally.

2. The hypersurfaces of $Z, E \cap Z=\{H \cap Z \mid H \in E\}$, have only normal crossings.

Then we define the coefficient ideal

$$
\operatorname{Coeff}_{Z}(J)=\sum_{i=0}^{b-1}\left(\Delta^{i}(J) \mathcal{O}_{Z}\right)^{\frac{b !}{b-i}} \subset \mathcal{O}_{Z}
$$

If the coefficient ideal is non-zero, we also define the basic object

$$
\left(Z,\left(\operatorname{Coeff}_{Z}(J), b !\right), E \cap Z\right) .
$$

Remark 13.11. Note that the conditions required in Definition 13.10 are trivially satisfied if $E=\emptyset$. We will show that the ideals $\mathcal{A}_{\alpha}=\operatorname{Coeff}_{\widetilde{W}_{\alpha}}\left(J_{\alpha}\right)$ will satisfy the conditions in Proposition 13.3, with $e=b$ !.

Example 13.12. Let $W=\mathbb{A}_{\mathbf{k}}^{3}$ and consider the ideal $J=\left\langle z^{2}+x^{3} y^{3}\right\rangle$, generated by an equation expressed in a Tschirnhausen form (following Abhyankar). Then

$$
\Delta(J)=\left\langle z^{2}+x^{3} y^{3}, 2 z, 3 x^{2} y^{3}, 3 x^{3} y^{2}\right\rangle,
$$

and if the characteristic of $\mathbf{k}$ is not two, then $\Delta^{2}(J)=\mathcal{O}_{W}$. Note that

$$
\operatorname{Sing}(J, 2)=V(\Delta(J))=V(x, z) \cup V(y, z),
$$

and that ord $_{\mathcal{B}} \cong 1$. In fact, if $Z=V(\langle z\rangle)$ and $\mathcal{A}=\left\langle\left(x^{3} y^{3}\right)\right\rangle$, then

$$
(W,(J, 2), \emptyset) \cong(Z,(\mathcal{A}, 2), \emptyset),
$$


since the equation has a Tschirnhausen form. So $(W,(J, 2), \emptyset)$ has a structure of two-dimensional basic object (note that $\mathcal{A}=\operatorname{Coeff}_{Z}(J)$ ). Although we will not prove this equivalence here (see Proposition 19.7), we already point out that this is the key step in the induction argument behind the proof of Proposition 13.3 and hence in the proof of Theorem 3.10 (we refer the reader to Section 19 for a complete proof that an equivalence of the form (13.12.1) holds in general).

Remark 13.13. Equivalence (13.12.1) can be established thanks to the fact that $Z \subset W$ is a smooth hypersurface defined by an ideal $I(Z) \subset \Delta^{b-1}(J)$. In general, given a basic object $\mathcal{B}=(W,(J, b), E)$ such that $\operatorname{ord}_{\mathcal{B}} \cong 1$, we will not be able to find a smooth hypersurface $\widetilde{W} \subset W$ such that globally,

$$
\mathcal{I}(\widetilde{W}) \subset \Delta^{b-1}(J) .
$$

We shall prove that a smooth hypersurface $\widetilde{W}$ that fulfills condition (13.13.1), also has the property that

$$
(W,(J, b), E) \subset(W,(\mathcal{I}(\widetilde{W}), 1), E),
$$

(see 19.3). Furthermore, we shall proof that if $\mathcal{B}=(W,(J, b), E) \longleftarrow \mathcal{B}_{1}=$ $\left(W_{1},\left(J_{1}, b\right), E_{1}\right)$ is a transformation as in 3.6 or as in 3.12 , then $\mathcal{I}(\widetilde{W})_{1} \subset$ $\Delta^{b-1}\left(J_{1}\right)$. Here $\mathcal{I}(\widetilde{W})_{1}=\mathcal{I}\left(\widetilde{W}_{1}\right)$ where $\widetilde{W}_{1}$ is the strict transform of $\widetilde{W}$ in the first case (see Remark 3.9 (2)), and the pull-back in the second case. So, in either case $\Delta^{b-1}\left(J_{1}\right)$ has order at most one, so $\operatorname{Sing}(J, b)=\emptyset$ or $\operatorname{ord}_{\mathcal{B}_{1}} \cong 1$.

The key point for the proof of Proposition 13.3 is the inclusion (13.13.2). The role of (13.13.1) is technical, but provides a way of finding hypersurfaces for which 13.13.2 holds. This fact naturally leads us to the notion of general basic object, which will be introduced in Section 14. The following simple example will illustrate this notion, with $b=1$, and hence $\Delta^{b-1}(J)=J$.

Example 13.14. Let $W$ be smooth of dimension $d$, and let $X \subset W$ be a smooth curve. One can always define an open covering of $W,\left\{U_{\alpha}\right\}_{\alpha \in \Lambda}$, such that the restriction of the curve, $X_{\alpha}=X \cap U_{\alpha}$, is a complete intersection on each open subset $U_{\alpha}$, i.e.,

$$
\mathcal{I}\left(X_{\alpha}\right)=\left\langle f_{1}, \ldots, f_{d-1}\right\rangle=J_{\alpha}
$$

Let $E=\emptyset$ and let $\left(U_{\alpha},\left(J_{\alpha}, 1\right), E_{\alpha}\right)$ be the restriction of $(W,(J=\mathcal{I}(X), 1), E)$ to $U_{\alpha}$ and define $Z_{\alpha}=V\left(f_{1}\right)$. Then

$$
\left(U_{\alpha},\left(J_{\alpha}, 1\right), E_{\alpha}\right) \subset\left(U_{\alpha},\left(f_{1}, 1\right), E_{\alpha}\right),
$$

and

$$
\left(U_{\alpha},\left(J_{\alpha}, 1\right), \emptyset\right) \cong\left(Z_{\alpha},\left(\operatorname{Coeff}\left(J_{\alpha}\right), 1\right), \emptyset\right)
$$


As in Example 12.10, both basic objects describe the same closed set after any sequence of transformations. In particular, a resolution of the basic object $\left(U_{\alpha},\left(J_{\alpha}, 1\right), \emptyset\right)$ is equivalent to a resolution of $\left(Z_{\alpha},\left(\operatorname{Coeff}\left(J_{\alpha}\right), 1\right), \emptyset\right)$. The advantage of working with the second basic object is that it is a $(d-1)$ dimensional basic object. This already illustrates that our form of induction on the dimension is of local nature, and also leads to the consideration of closed sets which are locally described by basic objects (see Section 14 where the notion of general basic objects is introduced).

Remark 13.15. Note that in Example 13.14 the closed sets $\operatorname{Sing}\left(\operatorname{Coeff}\left(J_{\alpha}\right), 1\right)$ patch to the curve $X=\operatorname{Sing}(J, 1)$.

Example 13.12 illustrates why we require that the characteristic of the underlying field be zero, at least if we want condition (d) of Remark 13.9 among the equivalent conditions in case $\operatorname{ord}_{\mathcal{B}} \cong 1$. It is actually this point which imposes the constraint on the characteristic for the resolution of singularities of algebraic varieties, because it is not possible, in general, to use the argument of reduction in the dimension that we are using here.

Remark 13.16. Set $W \longleftarrow W_{1}$ a monoidal transformation with center $C$, let $H_{1}$ be the exceptional locus, and let $J \subset \mathcal{O}_{W}$ be a sheaf of ideals with order $b$ along points in $C$. Then $J \mathcal{O}_{W_{1}}=I\left(H_{1}\right)^{b} \cdot J_{1}$ and the order of $J_{1}$ is at most $b$ at the exceptional points of $W_{1}$ (in $H_{1} \subset W_{1}$ ). This is quite a general result, but in our context (over a field of characteristic zero) it also follows from Remark 13.13: $\Delta^{b-1}(J)$ has order one along the points in $C$, and hence, as indicated in Remark 13.13, $\Delta^{b-1}\left(J_{1}\right)$ has at most order one along exceptional points; so the claim on $J_{1}$ follows from 13.7, P2).

\section{General basic objects and the main inductive theorem: Theorem 14.8}

In the previous section we have already observed the need to generalize the notion of basic object in order to use an inductive argument for the proof of Theorem 3.10. This leads us to the notion of general basic objects which will be developed in this section. Inclusion, intersection and equivalence of basic objects will naturally extend to this new context, as well as the notion of equivariant functions (see Proposition 14.7). At the end of this section we extend Theorem 3.10, in terms of general basic objects, with a constructive formulation (3.11), in Theorem 14.8. The proof of Theorem 14.8 will be developed in Sections 15 and 16. Finally, in Section 17, we introduce the functions $f_{i}^{d}$ so that the resolution of basic objects, provided by Theorem 14.8, coincides with that defined by the algorithm described in Definition 5.5. 
Definition 14.1. A d-dimensional general basic object over a pair $(W, E)$ consists of an open covering of $W,\left\{U_{\alpha}\right\}_{\alpha \in \Lambda}$ such that if $\left(U_{\alpha}, E_{\alpha}\right)$ is the restriction of $(W, E)$ to $U_{\alpha}$, then we have:

(i) A collection of basic objects. For every $\alpha \in \Lambda$ there is a closed and smooth $d$-dimensional subscheme $\widetilde{W}_{\alpha} \subset U_{\alpha}$, which intersects transversally all hypersurfaces of $E_{\alpha}$ defining a pair $\left(\widetilde{W}_{\alpha}, \widetilde{E}_{\alpha}\right)$, and a basic object

$$
\left(\widetilde{W}_{\alpha},\left(B_{\alpha}, d_{\alpha}\right), \widetilde{E}_{\alpha}\right) \text {. }
$$

Obviously, for each index $\alpha$ the closed set

$$
\operatorname{Sing}\left(B_{\alpha}, d_{\alpha}\right) \subset U_{\alpha}
$$

is locally closed in $W$ (compare to conditions in 13.10).

(ii) A patching condition. There is a closed subset $F \subset W$ such that

$$
F \cap U_{\alpha}=\operatorname{Sing}\left(B_{\alpha}, d_{\alpha}\right)
$$

for every $\alpha \in \Lambda$.

(iii) Stability of patching (I). Let

$$
(W, E) \longleftarrow\left(W_{1}, E_{1}\right)
$$

be a permissible transformation with center $Y \subset F$, let $\left\{U_{\alpha, 1}\right\}$ be the pullback of $\left\{U_{\alpha}\right\}_{\alpha \in \Lambda}$ to $W_{1}$, and for each $\alpha \in \Lambda$ let

$$
\left(\widetilde{W}_{\alpha},\left(B_{\alpha}, d_{\alpha}\right), \widetilde{E}_{\alpha}\right) \longleftarrow\left(\widetilde{W}_{\alpha, 1},\left(B_{\alpha, 1}, d_{\alpha}\right), \widetilde{E}_{\alpha, 1}\right) .
$$

be the corresponding transformation of basic objects. Then there is a closed set $F_{1} \subset W_{1}$ so that

$$
F_{1} \cap U_{\alpha, 1}=\operatorname{Sing}\left(B_{\alpha, 1}, d_{\alpha}\right)
$$

for each index $\alpha \in \Lambda$.

(iv) Stability of patching (II). Let $W \leftarrow W_{1}$ be a projection and let

$$
(W, E) \longleftarrow\left(W_{1}, E_{1}\right)
$$

be the corresponding transformation of pairs as defined in 3.12. Let $\left\{U_{\alpha, 1}\right\}$ be the pullback of $\left\{U_{\alpha}\right\}_{\alpha \in I}$ to $W_{1}$, and for each $\alpha \in \Lambda$ let

$$
\left(\widetilde{W}_{\alpha},\left(B_{\alpha}, d_{\alpha}\right), \widetilde{E}_{\alpha}\right) \longleftarrow\left(\widetilde{W}_{\alpha, 1},\left(B_{\alpha, 1}, d_{\alpha}\right), \widetilde{E}_{\alpha, 1}\right) .
$$


be the transformation of basic objects as defined in 3.12. Then there is a closed set $F_{1} \subset W_{1}$ such that

$$
F_{1} \cap U_{\alpha, 1}=\operatorname{Sing}\left(B_{\alpha, 1}, d_{\alpha}\right)
$$

for each index $\alpha \in \Lambda$.

(v) Stability of patching (III). The patching condition defined in (iii) and (iv) holds after any sequence of transformations: Given a sequence of transformations of pairs,

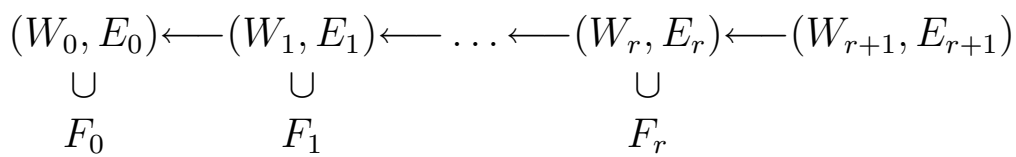

where for $i=0,1, \ldots, r, W_{i+1} \rightarrow W_{i}$ is defined either by:

(1) blowing up at centers $Y_{i}$, permissible for the pair $\left(W_{i}, E_{i}\right)$, and $Y_{i}$ included in the inductively defined closed sets $F_{i} \subset W_{i}$, or

(2) a projection $p: W_{i+1} \rightarrow W_{i}$,

there is an open covering $\left\{U_{\alpha, r+1}\right\}$ of $W_{r+1}$ (the pull back of $\left\{U_{\alpha}\right\}$ ), a sequence of transformations of basic objects,

$$
\begin{aligned}
\left(\widetilde{W}_{\alpha},\left(B_{\alpha}, d_{\alpha}\right), \widetilde{E}_{\alpha}\right) \longleftarrow & \left(\widetilde{W}_{\alpha, 1},\left(B_{\alpha, 1}, d_{\alpha}\right), \widetilde{E}_{\alpha, 1}\right) \longleftarrow \\
& \cdots \longleftarrow\left(\widetilde{W}_{\alpha, r+1},\left(B_{\alpha, r+1}, d_{\alpha}\right), \widetilde{E}_{\alpha, r+1}\right)
\end{aligned}
$$

and a closed set $F_{r+1} \subset W_{r+1}$, such that for each $\alpha \in \Lambda$,

$$
F_{r+1} \cap U_{\alpha, r+1}=\operatorname{Sing}\left(B_{\alpha, r+1}, d_{\alpha}\right) .
$$

(vi) Restriction to open sets. If $V \subset W$ is an open set, consider the restriction of all data to $V$ : The open covering $\left\{U_{\alpha} \cap V\right\}_{\alpha \in \Lambda}$, the basic objects $\left(\widetilde{W}_{\alpha},\left(B_{\alpha}, d_{\alpha}\right), \widetilde{E}_{\alpha}\right)_{V}$ and the closed set $F_{V}=F \cap V$. Then we require that all properties (i), (ii), (iii) (iv) and (v) hold for the restriction (see Remark 4.5).

A general basic object will be denoted by $(\mathcal{F},(W, E))$, the restriction to an open set $V$ will be denoted by $\left(\mathcal{F}_{V},\left(V, E_{V}\right)\right)$, and we will write a sequence of transformations and projections as

$$
\left(\mathcal{F}_{0},\left(W_{0}, E_{0}\right)\right) \longleftarrow \ldots \longleftarrow\left(\mathcal{F}_{r},\left(W_{r}, E_{r}\right)\right) \longleftarrow\left(\mathcal{F}_{r+1},\left(W_{r+1}, E_{r+1}\right)\right)
$$


Remark 14.2. If $(\mathcal{F},(W, E))$ is a general $d$-dimensional basic object, note that $d$ can be different from $\operatorname{dim} W$. A basic object $(W,(J, b), E)$ defines a general basic object $(\mathcal{F},(W, E))$, with the trivial open covering and in this case $d=\operatorname{dim} W$. A general basic object can be described by giving two different open coverings. What is important here are the closed sets $F$ that it defines. That is why in the notation for general basic objects $(\mathcal{F},(W, E))$ there is no reference to the open covering which appears in the definition (see Definition 14.5, where the notion of isomorphism of general basic objects is stated).

Example 14.3. Example 13.14 illustrates what a $d$-dimensional general basic object can be. In this case, the collection of locally closed basic objects is given by

$$
\left(U_{\alpha},\left(J_{\alpha}, 1\right), E_{\alpha}\right),
$$

and the closed subset $F \subset W$ of condition (ii) in Definition 14.1 is $X$. Now, consider a sequence of transformations of pairs,

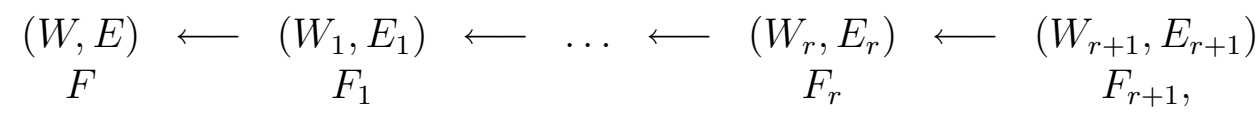

where each transformation is either as in Definition 14.1 (iii) or as in Definition 14.1 (iv). Then $F_{r+1}$ is either the strict transform of $F_{r}$, in the first case (this is a particular feature discussed in $3.9(2)$ ), or the pull-back of $F_{r}$ in $W_{r+1}$, in the second case.

Most definitions on basic objects extend naturally to general basic objects, and we extend only some of them. In Proposition 14.7 we extend the two equivariant functions introduced in Section 6.

Definition 14.4. A resolution of a general basic object $\left(\mathcal{F}_{0},\left(W_{0}, E_{0}\right)\right)$ is a sequence of transformations as in (14.1.2) which fulfills the following two conditions:

(i) The sequence involves only transformations as the ones introduced in Definition 14.1 (iii).

(ii) The closed set $F_{r+1}$ is empty.

Note that if $\left\{U_{\alpha}\right\}$ is an open covering of $W$ as in Definition 14.1, then for any $\alpha$ we obtain a resolution of the basic object $\left(\widetilde{W}_{\alpha},\left(B_{\alpha}, d_{\alpha}\right), \widetilde{E}_{\alpha}\right)$ in the sense of Definition 3.8. 
Definition 14.5. Let $\left(\mathcal{F}_{0},\left(W_{0}, E_{0}\right)\right)$ and $\left(\mathcal{F}_{0}{ }^{\prime},\left(W_{0}^{\prime}, E_{0}^{\prime}\right)\right)$ be two general basic objects and let

$$
\Theta:(W, E) \rightarrow\left(W^{\prime}, E^{\prime}\right)
$$

be an isomorphism of pairs (4.1). We will say that $\Theta$ induces an isomorphism of general basic objects,

$$
\Theta:\left(\mathcal{F}_{0},\left(W_{0}, E_{0}\right)\right) \rightarrow\left(\mathcal{F}_{0}{ }^{\prime},\left(W_{0}^{\prime}, E_{0}^{\prime}\right)\right),
$$

if the following conditions hold:

(i) The isomorphism $\Theta: W \rightarrow W^{\prime}$ induces an isomorphism of the closed subsets defined by the two general basic objects

$$
\Theta: F \cong F^{\prime}
$$

(ii) If

$$
\left(\mathcal{F}_{0},\left(W_{0}, E_{0}\right)\right) \longleftarrow \ldots \longleftarrow\left(\mathcal{F}_{r-1},\left(W_{r-1}, E_{r-1}\right)\right) \longleftarrow\left(\mathcal{F}_{r},\left(W_{r}, E_{r}\right)\right) ;
$$

is sequence of transformations of general basic objects, and

$$
(W, E) \longleftarrow\left(W_{1}, E_{1}\right) \longleftarrow \ldots \longleftarrow\left(W_{k}, E_{k}\right)
$$

is the corresponding sequence of transformation of pairs, then:

(a) The sequence induced by $\Theta$ as in Remark 4.4, say

$$
\left(W^{\prime}, E^{\prime}\right) \longleftarrow\left(W_{1}^{\prime}, E_{1}^{\prime}\right) \longleftarrow \ldots \longleftarrow\left(W_{k}^{\prime}, E_{k}^{\prime}\right),
$$

defines a sequence of transformation of general basic objects,

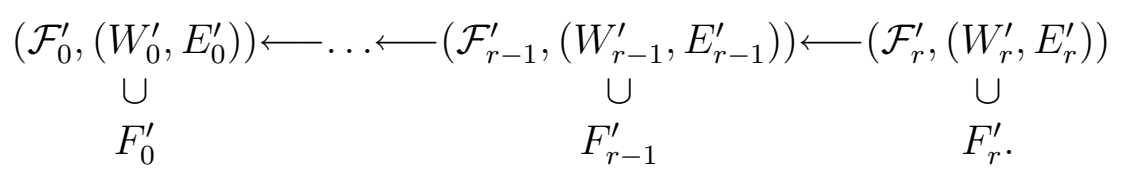

(b) The isomorphisms $\Theta_{i}:\left(W_{i}, E_{i}\right) \longrightarrow\left(W_{i}^{\prime}, E_{i}^{\prime}\right)$, presented in Definition 4.4, induce an isomorphism of the closed subsets defined by the general basic objects, i.e., $\Theta_{i}\left(F_{i}\right)=F_{i}^{\prime}$ for $i=0,1, \ldots, r+1$.

(iii) For any open set $U \subset W_{0}$, set $U^{\prime}=\Theta(U)$ and consider the restrictions $\left(\mathcal{F}_{0, U},\left(U, E_{0, U}\right)\right)$ and $\left(\mathcal{F}_{0, U^{\prime}}^{\prime},\left(U^{\prime}, E_{0, U^{\prime}}^{\prime}\right)\right)$ (see Definition 14.1 (vi)). Then we require that properties (i) and (ii) hold for the restrictions. 
Definition 14.6. Assume that for any general basic object $(\mathcal{F},(W, E))$ there is an upper semi-continuous function

$$
f_{\mathcal{F}}: F \rightarrow(T, \geq)
$$

associated to it, this is what we call a family of functions with values on $T$. We will say that the family of functions $f_{\mathcal{F}}$ is equivariant if:

(i) For any isomorphism of general basic objects $\Theta:(\mathcal{F},(W, E)) \rightarrow$ $\left(\mathcal{F}^{\prime},\left(W^{\prime}, E^{\prime}\right)\right)$ we have that

$$
f_{\mathcal{F}^{\prime}} \circ \Theta=f_{\mathcal{F}}
$$

(ii) If $U \subset W$ is an opens set, let $\left(\mathcal{F}_{U},\left(U, E_{U}\right)\right)$ be the restriction as in Definition 14.1 (vi). Then the function $f_{\mathcal{F}_{U}}$ is the restriction of $f_{\mathcal{F}}$.

Proposition 14.7. Let $(\mathcal{F},(W, E))$ be a general basic object, let $\left\{U_{\alpha}\right\}_{\alpha \in \Lambda}$ be the corresponding open covering of $W$ and let $\left(\widetilde{W}_{\alpha},\left(B_{\alpha}, d_{\alpha}\right), \widetilde{E}_{\alpha}\right)$ be the collection of d-dimensional basic objects associated to $(\mathcal{F},(W, E))$. Then the functions

$$
\operatorname{ord}_{\alpha}^{d}: \operatorname{Sing}\left(B_{\alpha}, d_{\alpha}\right) \rightarrow \mathbb{Q} \quad \text { and } \quad n_{\alpha}^{d}: \operatorname{Sing}\left(B_{\alpha}, d_{\alpha}\right) \rightarrow \mathbb{Z}
$$

patch so as to define equivariant functions

$$
\operatorname{ord}_{\mathcal{F}}^{d}: F \rightarrow \mathbb{Q} \quad \text { and } \quad n_{\mathcal{F}}^{d}: F \rightarrow \mathbb{Z}
$$

which verify the requirements of Definition 14.6.

We sketch the proof below, which parallels the proof of Lemma 6.7 for basic objects, and we refer to Section 21 for technical details.

Proof: Because of the way they are defined, any transformation of general basic objects

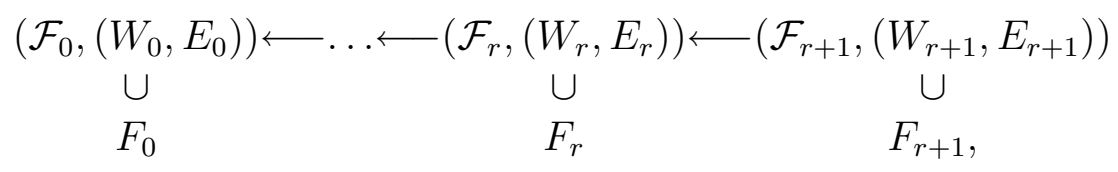

induces, for each index $\alpha$, a sequence of transformations of basic objects

$$
\begin{aligned}
\left(\widetilde{W}_{\alpha},\left(B_{\alpha}, d_{\alpha}\right), \widetilde{E}_{\alpha}\right) \longleftarrow & \left(\left(\widetilde{W}_{\alpha}\right)_{1},\left(\left(B_{\alpha}\right)_{1}, d_{\alpha}\right),\left(\widetilde{E}_{\alpha}\right)_{1}\right) \longleftarrow \cdots \\
& \cdots \longleftarrow\left(\left(\widetilde{W}_{\alpha}\right)_{r+1},\left(\left(B_{\alpha}\right)_{r+1}, d_{\alpha}\right),\left(\widetilde{E}_{\alpha}\right)_{r+1}\right) .
\end{aligned}
$$

In general, an arbitrary sequence of transformations over $\left(\widetilde{W}_{\alpha},\left(B_{\alpha}, d_{\alpha}\right), \widetilde{E}_{\alpha}\right)$, will not give rise to a sequence of transformations of the general basic object. However, this will be so if we fix $\alpha$ together with a point $x_{0} \in \operatorname{Sing}\left(B_{\alpha}, d_{\alpha}\right)$, and take the sequence (14.7.2) to be an arbitrary $x_{0}$-extendable sequence (6.4). 
So assume now that the sequence (14.7.1) induces an $x_{0}$-extendable sequence (14.7.2). In this case, for any index $j=0,1, \ldots, r$, there is an identification of fibers,

$$
\left(\operatorname{Sing}\left(\left(B_{\alpha, j}\right), d_{\alpha}\right)\right)_{x_{0}}=\left(F_{j}\right)_{x_{0}} .
$$

The characterization of the rational number $\operatorname{ord}_{\alpha}^{d}\left(x_{0}\right)\left(\mathrm{d}=\operatorname{dim}\left(\widetilde{W}_{\alpha}\right)\right)$ in terms of the dimensions of these closed sets (see Lemma 6.7) shows that, for $x_{0} \in F_{0} \cap U_{\alpha} \cap U_{\beta}, \operatorname{ord}_{\alpha}^{d}\left(x_{0}\right)=\operatorname{ord}_{\beta}^{d}\left(x_{0}\right)$. In particular $\operatorname{ord}_{\mathcal{F}}^{d}$ is well defined (see also Proposition 21.1). The property of equivariance of the function $\operatorname{ord}_{\mathcal{F}}^{d}$ follows now from (6.7.2).

As for the function $n_{\mathcal{F}}^{d}$, note that it is well defined by the very definition of general basic object; the same can be said about the property of equivariance. In fact any isomorphism

$$
\Theta:\left(\mathcal{F}_{0},\left(W_{0}, E_{0}\right)\right) \rightarrow\left(\mathcal{F}_{0}{ }^{\prime},\left(W_{0}^{\prime}, E_{0}^{\prime}\right)\right)
$$

defines an isomorphism

$$
\Theta_{0}:\left(W_{0}, E_{0}\right) \rightarrow\left(W_{0}^{\prime}, E_{0}^{\prime}\right)
$$

and the functions $n_{\mathcal{F}}$ and $n_{\mathcal{F}^{\prime}}$ are defined in terms of these pairs.

Theorem 14.8. (Theorem (d)) To each d-dimensional general basic object $\left(\mathcal{F}_{0},\left(W_{0}, E_{0}\right)\right)$ we can attach a resolution $R_{\mathcal{F}_{0}}$ in the sense of Definition 14.4,

$$
\begin{array}{ccc}
\left(\mathcal{F}_{0},\left(W_{0}, E_{0}\right)\right) \longleftarrow \ldots \longleftarrow \\
F_{0} & \cup & \left.\mathcal{F}_{r},\left(W_{r}, E_{r}\right)\right) \longleftarrow\left(\mathcal{F}_{r+1},\left(W_{r+1}, E_{r+1}\right)\right) \\
F_{r} & F_{r+1}=\emptyset,
\end{array}
$$

which has the following equivariance property: If $\Theta:\left(\mathcal{F}_{0},\left(W_{0}, E_{0}\right)\right) \rightarrow$ $\left(\mathcal{F}_{0}^{\prime},\left(W_{0}^{\prime}, E_{0}^{\prime}\right)\right)$ is an isomorphism of general basic objects, and if

$$
\begin{gathered}
R_{\mathcal{F}_{0}}:\left(\mathcal{F}_{0},\left(W_{0}, E_{0}\right)\right) \longleftarrow \ldots \longleftarrow\left(\mathcal{F}_{r-1},\left(W_{r-1}, E_{r-1}\right)\right) \longleftarrow \\
F_{0} \\
F_{r-1}
\end{gathered}
$$

and

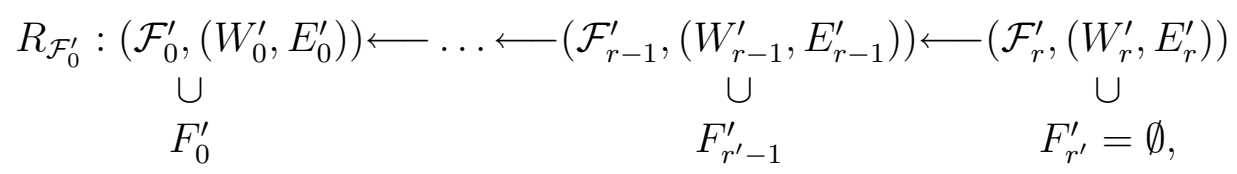

are the corresponding resolutions associated to $\mathcal{F}_{0}$ and $\mathcal{F}_{0}^{\prime}$, then there is a lifting of $\Theta$ to isomorphisms

$$
\Theta_{i}:\left(\mathcal{F}_{i},\left(W_{i}, E_{i}\right)\right) \rightarrow\left(\mathcal{F}_{i}^{\prime},\left(W_{i}^{\prime}, E_{i}^{\prime}\right)\right), \quad\left(\text { so } \Theta_{i}\left(F_{i}\right)=F_{i}^{\prime}\right) \text { for } i=1, \ldots, r,
$$

or, in other words, both resolutions are related by an isomorphism as in Definition 14.5 (ii). 
We will show later, that the resolutions $R_{\mathcal{F}_{0}}$, to be constructed here, will also be that defined by the functions $f_{i}^{d}$ that appear in Definition 5.5. The fact that the definition of the functions $f_{i}^{d}$ is given later, in section 17 , is due to the fact that the invariants involved in the definition of such functions is largely motivated by our proof of 14.8 .

The proof of Theorem 14.8 will be presented through the next two sections: In Section 15 the theorem will be proven for the case of basic objects, and in Section 16 the general case will be treated. Since the proof is based in an inductive argument, we already show why Theorem 14.8 holds for 0-dimensional general basic objects: namely, in that in that case, each $\left(\widetilde{W}_{\alpha},\left(B_{\alpha}, d_{\alpha}\right), E_{\alpha}\right)$ is zero dimensional, so we can assume that each $\widetilde{W}_{\alpha}$ is a point, and hence, each $B_{\alpha}$ is a non-zero ideal in a field. Therefore, $\operatorname{Sing}\left(B_{\alpha}, d_{\alpha}\right)=\emptyset$, and hence, $F_{0}=\emptyset$.

\section{Proof of Theorem 14.8 for basic objects}

Given a basic object $\mathcal{B}=(W,(J, b), E)$, we will distinguish three cases:

- Case 1: $\operatorname{ord}_{\mathcal{B}} \cong 1$ and $E=\emptyset$

- Case 2: $\operatorname{ord}_{\mathcal{B}} \cong 1$ and $E \neq \emptyset$.

- Case 3: The general case: Proof of Theorem 14.8 for basic objects.

Cases 1 and 2 are not special cases: They appear as intermediate steps when proving the general case.

\section{Case 1.}

We will first study the case $B=(W,(J, b), \emptyset)$ such that $\operatorname{ord}_{B} \cong 1$, or a transform of this case. Induction in this case parallels the treatment of Tschirnhausen transformations (13.12). This is the simplest situation to be considered, but at the same time the most significant; in fact it shows why centers defined by our procedure are regular, and why the algorithm of resolution of basic objects is equivariant. The key point in this case is the fact that the closed set $\operatorname{Sing}(J, b)=V\left(\Delta^{b-1}(J)\right)$ is defined by an ideal of order at most one.

Proposition 15.1. Theorem 14.8 holds in Case 1.

To prove this statement we will need some auxiliary results: Lemmas 15.4 and 15.5. The proof of Proposition 15.1 will be given in 15.7. To illustrate the general idea behind this case, let us consider the following example: 
Example 15.2. Let $W=\operatorname{Spec}(k[x, y, z])$, let

$$
J=<z^{2}+y^{3} x^{2}>\subset \mathcal{O}_{W}=k[x, y, z],
$$

$E=\emptyset$ and consider the basic object $(W,(J, 2), E)$. In this case $F=$ $\operatorname{Sing}(J, 2)$ is a union of two lines, and therefore $R(1)(F)=\emptyset$ (see Definition 13.1). Note that $\langle z\rangle \in \Delta(J)$ (see (13.13.1)).

Let $\widetilde{W}=V(\langle z\rangle) \subset W$. By Proposition 19.7 we may consider the two dimensional basic object

$$
(\widetilde{W}, \mathcal{A}, \emptyset)=\left(\widetilde{W},\left(y^{3} x^{2}, 2\right), \emptyset\right) .
$$

This basic object defines the 2-dimensional structure associated to $(W,(J, 2)$, $E$ ) in the sense of 12.9 . In this case note that

$$
\operatorname{Sing}\left(<z^{2}+y^{3} x^{2}>, 2\right)=\operatorname{Sing}\left(y^{3} x^{2}, 2\right),
$$

and furthermore the equality holds after any sequence of transformations. For example, blow-up the origin in both cases and compare the closed sets defined by

$(W,(J, 2), \emptyset) \longleftarrow\left(W_{1},\left(J_{1}, 2\right), E_{1}\right)$ and $(\widetilde{W},(\mathcal{A}, 2), \emptyset) \longleftarrow\left(\widetilde{W}_{1},\left(\mathcal{A}_{1}, 2\right), E_{1}\right)$

Summarizing we have that:

- A resolution of $(W,(J, 2), \emptyset)$ induces a resolution of $(\widetilde{W},(\mathcal{A}, 2), \emptyset)$.

- A resolution of $\left(W,\left(J_{1}, 2\right), E_{1}\right)$ induces a resolution of $\left(\widetilde{W},\left(\mathcal{A}_{1}, 2\right), \widetilde{E}_{1}\right)$.

Remark 15.3. Note that:

(1) If $(W,(J, b), E)$ is such that ord $\cong 1$, so is any transform (see Remark 13.13).

(2) If there is an inclusion of basic objects,

$$
(W,(K, d), E) \subset(W,(J, b), E)
$$

and $(W,(J, b), E)$ is such that ord $\cong 1$, then same holds for $(W,(K, d), E)$. This follows from 12.7,

$$
(W,(K, d), E) \cap(W,(J, b), E) \cong(W,(K, d), E),
$$

and the fact that the function ord is equivariant (6.7). 
Lemma 15.4. Let $\mathcal{B}=(W,(J, b), \emptyset)$ be a basic object such that $\operatorname{ord}_{\mathcal{B}} \cong 1$. Set $F=\operatorname{Sing}(J, b)=V\left(\Delta^{b-1}(J)\right)$ and let $G=\operatorname{Sing}(J, b) \backslash R(1)(F)$ be the union of the irreducible components of $\operatorname{Sing}(J, b)$ of codimension greater than or equal to 2. Then:

(a) $R(1)(F)$ and $G$ are both open and closed in $F$.

(b) $R(1)(F)$ is either empty or regular.

(c) If $R(1)(F)$ is not empty, then it is a permissible center for $(W,(J, b), \emptyset)$ and if

$$
(W,(J, b), \emptyset) \longleftarrow\left(W_{1},\left(J_{1}, b\right), E_{1}\right)
$$

is the transformation with center $R(1)(F)$, then

$$
W_{1}=W \quad \text { and } \quad R(1)\left(F_{1}\right)=\emptyset .
$$

(d) If $R(1)(F)=\emptyset$ then the basic object $(W,(J, b), \emptyset)$ has a structure of $(d-1)$-dimensional general basic object.

Proof: Note that if $x \in R(1)$, then $J_{x}=<l^{b}>\subset \mathcal{O}_{W, x}$, where $l \in \mathcal{O}_{W, x}$ is an element of order one. This reduces to two observations:

(i) If an ideal of order one at $\mathcal{O}_{W, x}$ defines a hypersurface, the ideal must be principal and generated by an element of order one.

(ii) If $Z$ is a smooth irreducible hypersurface included in $\operatorname{Sing}(J, b)$, then the order of $J$ at the generic point if $Z$ is at least b, and $J=\mathcal{I}(Z)^{b} \cdot J^{\prime} \subset \mathcal{O}_{W}$.

These observations prove statements (a), (b) and (c). Statement (d) follows from Proposition 13.3, which we reformulate below for a sequence of transformations. Note that if $R(1)(F)=\emptyset$ then $\operatorname{Coeff}_{Z}(J) \neq 0$ (see Definition 13.10).

Lemma 15.5. Let $\mathcal{B}_{0}=\left(W_{0},\left(J_{0}, b\right), \emptyset\right)$ be a basic object such that ord $d_{\mathcal{B}_{0}} \cong 1$ and let

$$
B_{0}=\left(W_{0},\left(J_{0}, b\right), E_{0}=\emptyset\right) \longleftarrow \ldots \longleftarrow B_{k}=\left(W_{k},\left(J_{k}, b\right), E_{k}\right)
$$

be a sequence of transformations of basic objects. Then:

(a) $R(1)\left(\operatorname{Sing}\left(J_{k}, b\right)\right)$ is the strict transform of $R(1)\left(\operatorname{Sing}\left(J_{0}, b\right)\right)$.

(b) If $\left(W_{0},\left(J_{0}, b\right), E_{0}=\emptyset\right) \subset\left(W_{0},\left(\mathcal{I}\left(\widetilde{W_{0}}\right), 1\right), E_{0}=\emptyset\right)$ for some smooth hypersurface $\widetilde{W_{0}} \subset W_{0}$, and

$$
\left(W_{0},\left(\mathcal{I}\left(\widetilde{W_{0}}\right), 1\right), E_{0}=\emptyset\right) \longleftarrow \cdots \longleftarrow\left(W_{k},\left(\mathcal{I}\left(\widetilde{W_{k}}\right), 1\right), E_{k}\right)
$$


is the sequence induced by (15.5.1), then

$$
\left(W_{k},\left(J_{k}, b\right), E_{k}\right) \subset\left(W_{k},\left(\mathcal{I}\left(\widetilde{W_{k}}\right), 1\right), E_{k}\right)
$$

and $\mathcal{I}\left(\widetilde{W}_{k}\right)$ defines a smooth hypersurface $\widetilde{W}_{k} \subset W_{k}$ transversal to all hypersurfaces of $E_{k}$.

(c) If $U_{0, \alpha}$ and $\widetilde{W}_{0 \alpha}$ denote, respectively, the open covering of $W_{0}$ and the corresponding smooth hypersurfaces stated in Proposition 13.3, then for each index $\alpha$, sequence (15.5.1) induces a sequence

$$
\left(U_{0, \alpha},\left(J_{0, \alpha}, b\right), E_{0, \alpha}=\emptyset\right) \longleftarrow \ldots \longleftarrow\left(\left(U_{k, \alpha}\right),\left(J_{k, \alpha}, b\right),\left(E_{k, \alpha}\right)\right)
$$

the open subsets $\left(U_{k, \alpha}\right)$ define an open cover of $W_{k}$, and for each index $\alpha$, this sequence can be replaced by one in dimension $d-1$ (13.3 (b)) since

$$
\left(U_{k, \alpha},\left(J_{k, \alpha}, b\right), E_{k, \alpha}\right) \cong\left(\widetilde{W}_{k, \alpha},\left(\mathcal{A}_{\alpha}, e_{\alpha}\right), \bar{E}_{k, \alpha}\right)
$$

Proof: Part (b) follows from 12.2, and (c) follows from Proposition 13.3. Part (a) follows from the proof of Lemma 15.4.

Remark 15.6. If only monoidal transformations arise in sequence (15.5.1), then:

1) If $R(1)\left(\operatorname{Sing}\left(J_{k}, b\right)\right)=\emptyset$, then $\mathcal{B}_{k}=\left(W_{k},\left(J_{k}, b\right), E_{k}\right)$ has a $(d-1)$ dimensional structure (Lemma $15.4(\mathrm{~d}))$ ).

2) If $R(1)\left(\operatorname{Sing}\left(J_{k}, b\right)\right)=\emptyset$ and

$$
\mathcal{B}_{k}=\left(W_{k},\left(J_{k}, b\right), E_{k}\right) \cong \mathcal{B}_{k}^{\prime}=\left(W_{k}^{\prime},\left(J_{k}^{\prime}, b^{\prime}\right), E_{k}\right),
$$

then $\mathcal{B}_{k}^{\prime}$ has a $(d-1)$-dimensional structure (Lemma $\left.15.4(\mathrm{~d})\right)$.

3) Let $\left(W_{k},(\mathcal{C}, s), E_{k}\right) \subset \mathcal{B}_{k}$. If $R(1)(\operatorname{Sing}(\mathcal{C}, s))=\emptyset$ and

$$
\left(W_{k},(\mathcal{C}, s), E_{k}\right) \subset \mathcal{B}_{k}=\left(W_{k},\left(J_{k}, b\right), E_{k}\right)
$$

then $\left(W_{k},(\mathcal{C}, s), E_{k}\right)$ has a $(d-1)$-dimensional structure (Lemma $\left.15.4(\mathrm{~d})\right)$.

All three observations follow from the fact that isomorphisms stated in (15.5.3) require only inclusion (15.5.2) to hold. 
15.7. Proof of Proposition 15.1: Assume that $\mathcal{B}=(W,(J, b), E=\emptyset)$ is within Case 1. If $R(1)(\operatorname{Sing}(J, b))=\emptyset$, then we apply Lemma $15.4(\mathrm{~d})$, and the statement follows by induction on $d$; in fact we are assuming Theorem 14.8 for $(d-1)$-dimensional general basic objects.

If $R(1)(\operatorname{Sing}(J, b)) \neq \emptyset$, then by Lemma 15.4 (c), after blowing-up $R(1)(F)$ we may assume that the basic object has a structure of $(d-1)$-dimensional general basic object. Again we proceed by induction.

If $\Theta: \mathcal{B}=(W,(J, b), E=\emptyset) \cong \mathcal{B}^{\prime}=\left(W^{\prime},\left(J^{\prime}, b^{\prime}\right), E^{\prime}=\emptyset\right)$ is an isomorphism of basic objects, then $B^{\prime}$ is within case 1 , and there is an isomorphism $\Theta: W \rightarrow W^{\prime}$, mapping $F=\operatorname{Sing}(J, b)$ isomorphically to $F^{\prime}=\operatorname{Sing}\left(J^{\prime}, b^{\prime}\right)$. It is clear that for any such isomorphism $\Theta, \Theta(R(1)(F))=R(1)\left(F^{\prime}\right)$. Note also that if $R(1)(F)=\emptyset=R(1)\left(F^{\prime}\right)$ then $\Theta$ induces an isomorphism of the corresponding $(d-1)$-dimensional general basic objects (see Lemma $15.4(\mathrm{~d}))$.

By induction there is a well defined equivariant resolution $\mathcal{R}_{\mathcal{B}}$ for any $\mathcal{B}=$ $(W,(J, b), E)$ within Case 1 . Hence Theorem 14.8 holds within this case.

Remark 15.8. Fix $\mathcal{B}_{0}=\left(W_{0},\left(J_{0}, b\right), \emptyset\right)$ within Case 1 , and now consider any sequence of permissible monoidal transformations as in (15.5.1), not necessarily related to the resolution $\mathcal{R}_{\mathcal{B}_{0}}$ defined in 15.7. We claim now that the basic object $\mathcal{B}_{k}=\left(W_{k},\left(J_{k}, b\right), E_{k}\right)$ can also be considered and treated as in Case 1 (although $E_{k}$ is no longer empty), in the sense that we can attach a resolution to $\mathcal{B}_{k}$ by the same arguments. In fact, $F=\operatorname{Sing}\left(J_{0}, b\right)$ is a disjoint union of two closed sets $F=R(1)(F) \cup G$ (Lemma 15.4), and $\mathcal{B}_{0}$ has a structure of $d$-1-dimensional general basic object in an open neighborhood of $G$. Now set $F_{k}=\operatorname{Sing}\left(J_{k}, b\right)$ and the disjoint union $F_{k}=R(1)\left(F_{k}\right) \cup G_{k}$ as before. By Lemma 15.5 (a), $R(1)\left(F_{k}\right)$ is the strict transform of $R(1)(F)$; and locally at points of $G_{k}, \mathcal{B}_{k}$ inherits the $d$-1-dimensional structure of $\mathcal{B}_{0}$ locally at $G$. So the whole point is to note that $R(1)\left(F_{k}\right)$ is either empty or a permissible center (it has normal crossings with $E_{k}$ ), which follows from the fact that it is the strict transform of $R(1)(F)$, and all irreducible components of the centers are either disjoint or included in the intermediate strict transforms of this hypersurface.

\section{The general pattern of Cases 2 and 3}

15.9. Cases 2 and 3 follow a general pattern which we describe now: For each $d$-dimensional basic object $\mathcal{B}=(W,(J, b), E)$ we will define an upper semi-continuous function with values in a totally ordered set (a family in the sense of Definition 5.2),

$$
h=h_{\mathcal{B}}: \operatorname{Sing}(J, b) \rightarrow(T, \leq) .
$$

Note that $\underline{\operatorname{Max}}\left(h_{\mathcal{B}}\right) \subset \operatorname{Sing}(J, b)$. 
The function $h_{\mathcal{B}}$ is such that there will be a $d$-dimensional basic object $(W,(A, c), E)$ associated to $\underline{\operatorname{Max}}(h)$. In this case we will say that $h$ is associated to $\equiv(W,(A, c), E)$, and it has the following properties:

$\mathbf{P 1}$. There is an inclusion of basic objects as in (12.2)

$$
(W,(A, c), E) \subset(W,(J, b), E) .
$$

Hence, any sequence of transformations of basic objects,

$$
\left(W_{k},\left(A_{k}, c\right), E_{k}\right) \longleftarrow \ldots \longleftarrow\left(W_{N},\left(A_{N}, c\right), E_{N}\right)
$$

induces a sequence of transformations with the same centers

$$
\left(W_{k},\left(J_{k}, b\right), E_{k}\right) \longleftarrow \ldots \longleftarrow\left(W_{N},\left(J_{N}, b\right), E_{N}\right)
$$

and $\operatorname{Sing}\left(A_{s}, c\right) \subset \operatorname{Sing}\left(J_{s}, b\right)$ for $k \leq s \leq N$.

P2. If the basic object $\left(W_{k},\left(J_{k}, b\right), E_{k}\right)$ is within Case $i$, then $\left(W_{k},\left(A_{k}, c\right), E_{k}\right)$ will be within Case $i-1$ (for $i=2,3$ ), which is simpler.

P3. If sequence (15.9.3) follows from sequence (15.9.2) as in (P1), then we have that:

(a) If $\operatorname{Sing}\left(A_{N}, c\right) \neq \emptyset$, then $\max h_{k}=\max h_{k+1}=\cdots=\max h_{N-1}=$ $\max h_{N}$, and

$$
\operatorname{Sing}\left(A_{j}, c\right)=\underline{\operatorname{Max}}\left(h_{j}\right) \text { for } \quad k \leq j \leq N .
$$

(b) If $\operatorname{Sing}\left(A_{N}, c\right)=\emptyset$, then $\max h_{k}=\max h_{k+1}=\ldots=\max h_{N-1}>$ $\max h_{N}$, and

$$
\operatorname{Sing}\left(A_{j}, c\right)=\underline{\operatorname{Max}}\left(h_{j}\right) \text { for } k \leq j \leq N-1 .
$$

P4. If $\Theta:\left(W_{k},\left(J_{k}, b\right), E_{k}\right) \cong\left(W_{k}^{\prime},\left(J_{k}^{\prime}, b\right), E_{k}^{\prime}\right)$ is an isomorphism of basic objects and if $h_{k}, h_{k}^{\prime}$ are the corresponding upper semi-continuous functions

$$
h_{k}: \operatorname{Sing}\left(J_{k}, b\right) \longrightarrow(T, \geq) \quad \text { and } \quad h_{k}^{\prime}: \operatorname{Sing}\left(J_{k}^{\prime}, b\right) \longrightarrow(T, \geq),
$$

then $h_{k}^{\prime}(\Theta(\xi))=h_{k}(\xi)$ for all $\xi \in \operatorname{Sing}\left(J_{k}, b\right)$.

P5. Both the functions $h_{i}$, and the basic objects $\left(W_{i},\left(A_{i}, c\right), E_{i}\right)$ attached to it, are compatible with open restrictions of $\left(W_{i},\left(J_{i}, b\right), E_{i}\right)$ (see Definition 4.5). 
Remark 15.10. Note that:

A) A basic object provides a way of describing closed sets. We may think of $\left(W_{i},\left(A_{i}, c\right), E_{i}\right)$ as a basic object attached to the value max $h_{i}$; and property (P3) expresses this fact properly. It indicates, in particular, that if the sequence (15.9.2) is a resolution of $(W,(A, c), E)$, then in the sequence (15.9.3) we have that

$$
\max h_{k}=\max h_{k+1}=\cdots=\max h_{N-1}>\max h_{N},
$$

and $\operatorname{Sing}\left(A_{j}, c\right)=\underline{\operatorname{Max}}\left(h_{j}\right)$ for $k \leq j \leq N-1$. In other words, lowering $\max h_{k}$ is guaranteed by a resolution of the basic object $\left(W_{k},\left(A_{k}, c\right), E_{k}\right)$, attached to this maximum value.

B) If a sequence of transformations is defined (as in 3.6 and 3.12),

$$
\mathcal{B}=(W,(J, b), E) \longleftarrow \ldots \longleftarrow \mathcal{B}_{k}=\left(W_{k},\left(J_{k}, b\right), E_{k}\right),
$$

if

$$
\Theta: \mathcal{B}=(W,(J, b), E) \rightarrow \mathcal{B}^{\prime}=\left(W^{\prime},\left(J^{\prime}, b^{\prime}\right), E^{\prime}\right)
$$

is an isomorphism of basic objects, and if

$$
\mathcal{B}^{\prime}=\left(W^{\prime},\left(J^{\prime}, b^{\prime}\right), E^{\prime}\right) \longleftarrow \ldots \longleftarrow \mathcal{B}_{k}^{\prime}=\left(W_{k}^{\prime},\left(J_{k}^{\prime}, b^{\prime}\right), E_{k}^{\prime}\right)
$$

is the corresponding induced sequence, so that there are isomorphisms

$$
\Theta_{j}:\left(W_{j},\left(J_{j}, b\right), E_{j}\right) \cong\left(W_{j}^{\prime},\left(J_{j}^{\prime}, b^{\prime}\right), E_{j}^{\prime}\right),
$$

for $0 \leq j \leq k$ as in Definition 4.6 (ii), then (P3) and (P4) assert that $\Theta_{k}:\left(W_{k},\left(J_{k}, b\right), E_{k}\right) \cong\left(W_{k}^{\prime},\left(J_{k}^{\prime}, b^{\prime}\right), E_{k}^{\prime}\right)$ induces an isomorphism $\left(W_{k},\left(A_{k}, c\right), E_{k}\right) \cong\left(W_{k}^{\prime},\left(A_{k}^{\prime}, c^{\prime}\right), E_{k}^{\prime}\right)$.

C) The analog for general basic objects: If $\mathcal{B}=(\mathcal{F},(W, E))$ is now a general basic object and $\left\{U_{\alpha}\right\}_{\alpha \in I}$ is an open covering of $W$ as in Definition 14.1 (i), then for each index $\alpha$ and each basic object $\left(\widetilde{W}_{\alpha},\left(B_{\alpha}, d_{\alpha}\right), \widetilde{E}_{\alpha}\right)$ there is an upper semi-continuous function

$$
h_{\alpha}: \operatorname{Sing}\left(B_{\alpha}, d_{\alpha}\right) \longrightarrow(T, \leq) .
$$

If the collection of functions $\left\{h_{\alpha}\right\}$ patch so as to define a function,

$$
h: F \rightarrow(T, \leq)
$$

then the argument exhibit in (A) and (B) show that the different basic objects

$$
\left(\widetilde{W}_{\alpha},\left(A_{\alpha}, c_{\alpha}\right), \widetilde{E}_{\alpha}\right)
$$

(defined in terms of $\alpha$ ), define a general basic object attached to the value $\max h_{i}$. 
D) In our approach, the functions $h_{i}$ will be defined entirely in terms of the functions ord and $n$. So Proposition 14.7 guarantees that they define functions on general basic objects as required in (C).

\section{Case 2.}

Now we consider the case of a basic object $(W,(J, b), E)$ such that the function $\operatorname{ord}_{\mathcal{B}} \cong 1$, but where $E$ may not be empty.

Proposition 15.11. Theorem 14.8 holds for d-dimensional basic objects which are within Case 2.

The proof of this result, which will be stated in 15.17 , is based on the construction of suitable functions as indicated in 15.9. To illustrate the philosophy behind this case we present the following example:

Example 15.12. Let $W=\mathbb{A}_{\mathbf{k}}^{2}$. Set $J=\langle x-y\rangle$ the ideal of the diagonal $D$, and set $E=\left\{H_{1}, H_{2}\right\}$, where $H_{1}$ is the $x$-axis and $H_{2}$ the $y$-axis. The resolution of the basic object $(W,(J, 1), E)$ consists of two transformations:

$$
(W,(J, 1), E) \longleftarrow\left(W_{1},\left(J_{1}, 1\right), E_{1}\right) \longleftarrow\left(W_{2},\left(J_{2}, 1\right), E_{2}\right) .
$$

The first transformation is the blowing-up at the origin and the second is the blowing-up at $D^{\prime}$, where $D^{\prime}$ is the strict transform of the diagonal.

Now consider the automorphism $\Theta$ defined by the symmetry about the diagonal. The resolution is equivariant and the automorphism $\Theta$ lifts to automorphisms $\Theta_{1}$ in $\left(W_{1}, E_{1}\right)$ and $\Theta_{2}$ in $\left(W_{2}, E_{2}\right)$.

15.13. Let $\mathcal{B}=(W,(J, b), E)$ be a basic object such that $\operatorname{ord}_{\mathcal{B}} \cong 1$. Remark 15.3 asserts that for any sequence of transformations of basic objects

$$
\begin{aligned}
\mathcal{B}=(W,(J, b), E) \longleftarrow \mathcal{B}_{1}=\left(W_{1},\left(J_{1}, b\right), E_{1}\right) \longleftarrow \ldots \\
\ldots \longleftarrow \mathcal{B}_{k}=\left(W_{k},\left(J_{k}, b\right), E_{k}\right),
\end{aligned}
$$

then $\operatorname{Sing}\left(J_{k}, b\right)=\emptyset$ or $\operatorname{ord}_{\mathcal{B}_{i}} \cong 1$ for $1 \leq i \leq k$. Now consider the disjoint union

$$
E_{k}=E_{k}^{+} \cup E_{k}^{-}
$$

where $E_{k}^{-}$denotes the strict transform of hypersurfaces of $E$. Note that sequence (15.13.1) induces a sequence of transformations

$$
(W,(J, b), \emptyset) \longleftarrow\left(W_{1},\left(J_{1}, b\right), E_{1}^{+}\right) \longleftarrow \ldots \longleftarrow\left(W_{k},\left(J_{k}, b\right), E_{k}^{+}\right)
$$


Definition 15.14. With the notation introduced above, we define the upper semi-continuous function

$$
\begin{gathered}
n_{k}: \operatorname{Sing}\left(J_{k}, b\right) \rightarrow \mathbb{Z} \\
n_{k}(x)=\sharp\left\{H_{i} \in E_{k}^{-} \quad: x \in H_{i}\right\} .
\end{gathered}
$$

If $E_{k}^{-}=\left\{H_{1}, H_{2}, \ldots, H_{s}\right\}$, and $\max n_{k}=m$, we set

$$
\mathcal{P}_{k}=\mathcal{P}_{k}(m)=\prod_{i_{1}<\cdots<i_{m}}\left(\sum_{j=1}^{m} \mathcal{I}\left(H_{i_{j}}\right)\right)
$$

and define the basic objects

$$
\left(W_{k},\left(A_{k}, c\right), E_{k}^{+}\right)= \begin{cases}\left(W_{k},\left(J_{k}, b\right), E_{k}^{+}\right) \cap\left(W_{k},\left(\mathcal{P}_{k}, 1\right), E_{k}^{+}\right) & \text {if } m>0 \\ \left(W_{k},\left(J_{k}, b\right), E_{k}^{+}\right) & \text {if } m=0\end{cases}
$$

and

$$
\left(W_{k},\left(A_{k}, c\right), E_{k}\right)=\left\{\begin{array}{lll}
\left(W_{k},\left(J_{k}, b\right), E_{k}\right) \cap\left(W_{k},\left(\mathcal{P}_{k}, 1\right), E_{k}\right) & \text { if } & m>0 \\
\left(W_{k},\left(J_{k}, b\right), E_{k}\right) & \text { if } & m=0 .
\end{array}\right.
$$

Remark 15.15. Note that:

(a) By definition

$$
\left(W_{k},\left(A_{k}, c\right), E_{k}^{+}\right) \subset\left(W_{k},\left(J_{k}, b\right), E_{k}^{+}\right)
$$

(b) The function $n_{k}$ is associated to $\left(W_{k},\left(A_{k}, c\right), E_{k}\right)$ (linked by the five properties stated in 15.9). These properties will be discussed below.

(c) For any $\xi \in W_{k}$,

$$
\left(\mathcal{P}_{k}\right)_{\xi}=\mathcal{O}_{W_{k}} \quad \Longleftrightarrow \quad \xi \notin \underline{\operatorname{Max}} n_{k}
$$

In fact if $\xi \in \underline{\operatorname{Max}} n_{k}$ then

$$
\left(\mathcal{P}_{k}\right)_{\xi}=\sum_{j=1}^{m=\max \left(n_{k}\right)} \mathcal{I}\left(H_{i_{j}}\right)_{\xi} \quad \text { and } \quad \xi \in \bigcap_{j=1}^{m} H_{i_{j}} \quad H_{i_{j}} \in E_{k}^{-} .
$$

So $\underline{\operatorname{Max}} n_{k}=\operatorname{Sing}\left(A_{k}, c\right)$. Note now that the expressions in (15.14.3) and (15.14.2), as intersection of basic objects, are compatible with transformations in the sense of 12.7. This fact together with (a) prove that Property (P1) stated in 15.9 holds for the functions $n_{k}$. 
(d) If $C$ is a permissible center of $\left(W_{k},\left(\mathcal{P}_{k}, 1\right), E_{k}\right)$, for any hypersurface $H \in E_{k}^{-}$, and any irreducible component $C^{\prime}$ of $C$, either $C^{\prime} \subset H$ or $C^{\prime} \cap H=\emptyset$. This last observation and the following lemma will indicate that

$$
\left(W_{k},\left(A_{k}, c\right), E_{k}\right) \cong\left(W_{k},\left(A_{k}, c\right), E_{k}^{+}\right),
$$

in the sense of 4.6 , despite the fact that $E_{k} \neq E_{k}^{+}$. In fact the following lemma proves that both basic objects have the same sequences of transformations.

Lemma 15.16. Let $(W, E)$ be a pair, assume that $E$ is a disjoint union $E=$ $E^{+} \cup E^{-}$. If $C \subset W$ is a permissible center for $\left(W, E^{+}\right)$, and each irreducible component of $C$ is either disjoint from or included in hypersurfaces of $E^{-}$, then $C$ is permissible for $(W, E)$.

Proof: This follows from the definition of normal crossing (see Definition 2.1).

15.17. Proof of Proposition 15.11: First note that given a sequence of transformation of basic objects

$$
\mathcal{B}=\left(W_{0},\left(J_{0}, b\right), E_{0}\right) \longleftarrow \ldots \longleftarrow \mathcal{B}_{k}=\left(W_{k},\left(J_{k}, b\right), E_{k}\right),
$$

and isomorphisms

$$
\Theta: \mathcal{B}=(W,(J, b), E) \rightarrow \mathcal{B}^{\prime}=\left(W^{\prime},\left(J^{\prime}, b^{\prime}\right), E^{\prime}\right),
$$

sequence (15.17.1) induces a sequence

$$
\mathcal{B}^{\prime}=\left(W^{\prime},\left(J^{\prime}, b^{\prime}\right), E^{\prime}\right) \longleftarrow \ldots \longleftarrow \mathcal{B}_{k}^{\prime}=\left(W_{k}^{\prime},\left(J_{k}^{\prime}, b^{\prime}\right), E_{k}^{\prime}\right) .
$$

In particular, for $0 \leq j \leq k$, we have the isomorphisms

$$
\Theta_{j}:\left(W_{j}, E_{j}\right) \cong\left(W_{j}^{\prime}, E_{j}^{\prime}\right),
$$

as in Definition 4.6 (b). Now set

$$
E_{k}=E_{k}^{+} \cup E_{k}^{-} \quad \text { and } \quad E_{k}^{\prime}=E_{k}^{\prime+} \cup E_{k}^{\prime-},
$$

and consider the functions $n_{k}^{\prime}: \operatorname{Sing}\left(J_{k}^{\prime}, b\right) \rightarrow \mathbb{Z}$, and the corresponding basic objects

$$
\left(W_{k}^{\prime},\left(A_{k}^{\prime}, c^{\prime}\right), E_{k}^{\prime}\right)
$$

Clearly the functions $n_{k}$ are compatible with the expressions in (15.17.3) defined in terms of $E_{k}$ and $E_{k}^{\prime}$. Hence,

$$
n_{k}(x)=n_{k}^{\prime}\left(\Theta_{k}(x)\right) .
$$


Note that $\Theta_{k}$ defines an isomorphism

$$
\left(W_{k},\left(A_{k}, c\right), E_{k}\right) \cong\left(W_{k}^{\prime},\left(A_{k}^{\prime}, c^{\prime}\right), E_{k}^{\prime}\right)
$$

as can be checked directly from the construction of these basic object.

We are now ready to give a proof of Proposition 15.11. We begin by making some inductive assumptions on sequence (15.17.1): Assume first that sequence (15.17.1) is equivariant, and assume also that

$$
\max n_{0} \geq \max n_{1} \geq \ldots \geq \max n_{k} .
$$

Let $k_{0}$ be the index such that $\max n_{k_{0}-1}>\max n_{k_{0}}=\ldots=\max n_{k}$, and set $\left(W_{k_{0}},\left(A_{k_{0}}, c\right), E_{k_{0}}\right)$ as in (15.14.3).

By (15.15.2) a resolution of the basic object $\left(W_{k_{0}},\left(A_{k_{0}}, c\right), E_{k_{0}}\right)$ is equivalent to a resolution of $\left(W_{k_{0}},\left(A_{k_{0}}, c\right), E_{k_{0}}^{+}\right)$. We will show that

$$
\left(W_{k_{0}},\left(A_{k_{0}}, c\right), E_{k_{0}}^{+}\right)
$$

is within Case 1, in the extended form indicated in Remark 15.8 (see 15.9 (P2)). To check this, note that $\left(W_{0},\left(J_{0}, b\right), \emptyset\right)$ in $(15.13 .3)$ is within Case 1 , so the assertion follows from Remark 15.6.

Assume now, by induction on $k$, that the last $k-k_{0}$ transformations in sequence (15.17.1) are defined by the first $k-k_{0}$ steps of the equivariant resolution of $\left(W_{k_{0}},\left(A_{k_{0}}, c\right), E_{k_{0}}^{+}\right)$. We finally enlarge sequence $(15.17 .1)$ by the sequence induced by the equivariant resolution of $\left(W_{k_{0}},\left(A_{k_{0}}, c\right), E_{k_{0}}^{+}\right)$, as was indicated in $15.9(\mathrm{P} 1)$. This defines for some index $k^{\prime}>k$ an equivariant enlargement of sequence (15.17.1). Note that if $\max n_{k}=0$ this extension is already an equivariant resolution of $(W,(J, b), E)$. If not

$$
\max n_{k_{0}}=\cdots=\max n_{k}=\cdots=\max n_{k^{\prime}-1}>\max n_{k^{\prime}} .
$$

Repeating this procedure, ultimately $\max n_{k^{\prime}}=0$, and hence, we have constructed an equivariant resolution within this case, say

$$
R_{\mathcal{B}}=(W,(J, b), E) \longleftarrow\left(W_{1},\left(J_{1}, b\right), E_{1}\right) \longleftarrow \ldots \longleftarrow\left(W_{N},\left(J_{N}, b\right), E_{N}\right)
$$

together with a sequence $0=k_{0}<k_{1}<k_{2}<\cdots<k_{s} \leq N$, and

$$
\max n_{k_{i-1}}>\max n_{k_{i}}=\max n_{k_{i}+1}=\ldots=\max n_{k_{i+1}-1}>\max n_{k_{i+1}} \ldots
$$

This proves Proposition 15.11.

Note that in the Example 15.2, $\max n_{0}=2>\max n_{1}=1>\max n_{2}=0$. 


\section{Case 3: Proof of Theorem 14.8 for basic objects}

Our next objective is the following statement:

Proposition 15.18. Theorem 14.8 holds for d-dimensional basic objects.

We will start with an example to illustrate the invariants involved in the general case, and then we will introduce some auxiliary definitions and results that will be used in the proof of the proposition, which will be detailed in 15.25 .

Example 15.19. Let $W$ be the affine plane $\mathbb{A}_{k}^{2}$, let $J=<x^{3}, y^{4}>\subset k[x, y]$, set $b=2$, and consider the basic object $\mathcal{B}=(W,(J, 2), E=\emptyset)$. In this case $\operatorname{Sing}(J, 2)$ is the origin in $\mathbb{A}_{k}^{2}$, and $\operatorname{ord}_{\mathcal{B}}=3 / 2$. Now blow-up at the origin, $(W,(J, 2), \emptyset) \longleftarrow\left(W_{1},\left(J_{1}, 2\right), E_{1}=\left\{H_{1}\right\}\right)$.

Note that $J_{1} \subset \mathcal{O}_{W_{1}}$ is defined so that $J \mathcal{O}_{W_{1}}=\mathcal{I}\left(H_{1}\right)^{2} J_{1}$. However in this case, since the function ord is not equal to 1 , there is a new factorization of the form

$$
J_{1}=\mathcal{I}\left(H_{1}\right) \bar{J}_{1} .
$$

This factorization of $J_{1}$ does not hold for a basic object for which ord $\cong 1$, in fact $J_{1}=\bar{J}_{1}$ if ord $\cong 1$.

15.20. Given a sequence of transformation of basic objects

$$
\left(W_{0},\left(J_{0}, b\right), E_{0}\right) \longleftarrow \ldots \longleftarrow\left(W_{k},\left(J_{k}, b\right), E_{k}\right),
$$

consider the expression

$$
J_{k}=\mathcal{I}\left(H_{1}\right)^{\alpha(1)} \mathcal{I}\left(H_{2}\right)^{\alpha(2)} \ldots \mathcal{I}\left(H_{k}\right)^{\alpha(k)} \cdot \bar{J}_{k}
$$

which is unique, if we require that $\bar{J}_{k}$ does not vanish along (any component of) any $H_{i}$, arising from a previous transformation.

Remark 15.21. Let $b_{k}=\max \nu_{\bar{J}_{k}}$, where $\nu_{\bar{J}}$ is the numerator of the function introduced in 6.2. Note that $\operatorname{Sing}\left(\bar{J}_{k}, b_{k}\right)=\operatorname{Max} \nu_{\bar{J}_{k}}$. If the transformation is such that $C_{k} \subset \operatorname{Sing}\left(\bar{J}_{k}, b_{k}\right)$ then $\left(W_{k+1},\left(\overline{\bar{J}}_{k+1}, b_{k}\right), E_{k+1}\right)$ is the transform of the basic object $\left(W_{k},\left(\bar{J}_{k}, b_{k}\right), E_{k}\right)$. Moreover if $b_{k+1}=\max \nu_{\bar{J}_{k+1}}$ then $b_{k+1} \leq b_{k}$ (see Remark 13.16).

Definition 15.22. Given a sequence of transformations of basic objects as in (15.20.1), and expressions as in (15.20.2), we define the upper semicontinuous function:

$$
\begin{array}{cl}
\mathrm{w}-o r d_{k}: \operatorname{Sing}\left(J_{k}, b\right) & \rightarrow \mathbb{Q} \\
x & \rightarrow \mathrm{w}-\operatorname{ord}_{k}(x)=\nu_{x}\left(\bar{J}_{k}\right) / b .
\end{array}
$$


Remark 15.23. Given a sequence of transformations as in (15.20.1) note that:

(a) If sequence (15.20.1) is defined by blowing up centers $C_{i} \subset \underline{\text { Max } \text { w-ord }}{ }_{i}$, then by Remark 15.21

$$
\max \mathrm{w}-\operatorname{ord}_{0} \geq \max \mathrm{w}-\operatorname{ord}_{1} \geq \cdots \geq \max \mathrm{w}-\operatorname{ord}_{k} .
$$

(b) If $\max \mathrm{w}-\operatorname{ord}_{k}=0$ then

$$
J_{k}=\mathcal{I}\left(H_{1}\right)^{\alpha(1)} \mathcal{I}\left(H_{2}\right)^{\alpha(2)} \cdot \ldots \cdot \mathcal{I}\left(H_{k}\right)^{\alpha(k)} .
$$

(c) If $\operatorname{maxw}$-ord $d_{k}>0$ then the functions $\mathrm{w}$-ord $\mathrm{or}_{k}$ will play the role of the functions $h_{k}$ in (15.9.1). In fact if $\max \mathrm{w}-\operatorname{ord}_{k}=b_{k} / b>0$ for $0<b_{k}$ then we define:

$$
\text { (15.23.1) }\left(W_{k},\left(A_{k}, c\right), E_{k}\right)=\left(W_{k},\left(\bar{J}_{k}, b_{k}\right), E_{k}\right) \cap\left(W_{k},\left(J_{k}, b\right), E_{k}\right) .
$$

(d) The basic object $\left(W_{k},\left(\bar{J}_{k}, b_{k}\right), E_{k}\right)$ is such that ord $\cong 1$, so the same holds for the basic objects $\left(W_{k},\left(A_{k}, c\right), E_{k}\right)(15.3(2))$, and $\operatorname{Sing}\left(\bar{J}_{k}, b_{k}\right)=$

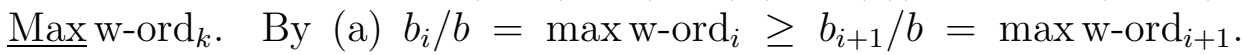
Note that if equality holds, then

$$
\left(W_{i+1},\left(\bar{J}_{i+1}, b_{i+1}\right), E_{i+1}\right)
$$

is the transform of $\left(W_{i},\left(\bar{J}_{i}, b_{i}\right), E_{i}\right)$.

(e) The function w-ord $\mathrm{w}_{k}$ is associated to $\left(W_{k},\left(A_{k}, c\right), E_{k}\right)$, and hence it satisfies the five properties stated in 15.9.

Lemma 15.24. Let $\Theta: \mathcal{B}=\left(W_{0},\left(J_{0}, b\right), E_{0}\right) \cong \mathcal{B}^{\prime}=\left(W_{0}^{\prime},\left(J_{0}^{\prime}, b^{\prime}\right), E_{0}^{\prime}\right)$ be an isomorphism of basic objects. Consider a sequence of transformations over $\mathcal{B}$,

$$
\left(W_{0},\left(J_{0}, b\right), E_{0}\right) \longleftarrow \ldots \longleftarrow\left(W_{k},\left(J_{k}, b\right), E_{k}\right),
$$

together with the induced sequence over $\mathcal{B}^{\prime}$ (as in Definition 4.6 (ii)),

$$
\mathcal{B}^{\prime}=\left(W_{0}^{\prime},\left(J_{0}^{\prime}, b^{\prime}\right), E_{0}^{\prime}\right) \longleftarrow \ldots \longleftarrow \mathcal{B}_{k}^{\prime}=\left(W_{k}^{\prime},\left(J_{k}^{\prime}, b^{\prime}\right), E_{k}^{\prime}\right),
$$

and let

$$
J_{k}=\mathcal{I}\left(H_{1}\right)^{\alpha(1)} \mathcal{I}\left(H_{2}\right)^{\alpha(2)} \cdot \ldots \cdot \mathcal{I}\left(H_{k}\right)^{\alpha(k)} \cdot \bar{J}_{k}
$$

and

$$
J_{k}^{\prime}=\mathcal{I}\left(H_{1}^{\prime}\right)^{\alpha^{\prime}(1)} \mathcal{I}\left(H_{2}^{\prime}\right)^{\alpha^{\prime}(2)} \cdot \ldots \cdot \mathcal{I}\left(H_{k}^{\prime}\right)^{\alpha^{\prime}(k)} \cdot \bar{J}_{k}^{\prime},
$$

be the factorizations of the ideals $J_{k}$ and $J_{k}^{\prime}$ as in 15.20. Set

$$
\xi_{k} \in \operatorname{Sing}\left(\bar{J}_{k}, b_{k}\right)=\underline{\operatorname{Max} \mathrm{w}-\operatorname{ord}_{k}}
$$

and $\Theta\left(\xi_{k}\right)=\xi_{k}^{\prime}$. Then: 
(i) For each $\xi_{k} \in \operatorname{Sing}\left(J_{k}, b\right)$ the rational numbers $\mathrm{w}-\operatorname{ord}_{k}\left(\xi_{k}\right)$, and the $\{\alpha(i) / b\}_{\{i=1, \ldots, k\}}$ corresponding to hypersurfaces containing $\xi_{k}$, can be expressed in terms of the rational numbers

$$
\left\{\operatorname{ord}_{\mathcal{B}_{0}}\left(\xi_{0}\right), \operatorname{ord}_{\mathcal{B}_{1}}\left(\xi_{1}\right), \ldots, \operatorname{ord}_{\mathcal{B}_{k}}\left(\xi_{k}\right)\right\}
$$

where $\xi_{i}$ denotes the image of $\xi_{k}$ in $W_{i}$.

(ii) For each $i=1, \ldots, k, \xi_{k} \in H_{i} \in E_{k}$ if and only if $\xi_{k}^{\prime} \in \Theta_{k}\left(H_{i}\right) \in E_{k}^{\prime}$, and in that case $\alpha(i) / b=\alpha^{\prime}(i) / b^{\prime}$.

(iii) The function $\mathrm{w}-\operatorname{ord}_{k}$ is equivariant, i.e., $\mathrm{w}-\operatorname{ord}_{k}\left(\xi_{k}\right)=\mathrm{w}-\operatorname{ord}_{k}^{\prime}\left(\xi_{k}^{\prime}\right)$.

Proof: We first indicate how to prove (i) by induction on $k$. If $k=0$, w-ord ${ }_{0}=\operatorname{ord}_{\mathcal{B}_{0}}$ so it follows from 6.7 .

If $k=1$, and $\xi_{1} \notin H_{1}$, then $\xi_{1}$ can be identified with $\xi_{0} \in W_{0}$, and w-ord $1\left(\xi_{1}\right)=\mathrm{w}-\operatorname{ord}_{0}\left(\xi_{0}\right)=\operatorname{ord}_{0}\left(\xi_{0}\right)$. If $k=1$ and $\xi_{1} \in H_{1}$, note that (as in Example 15.19) $\alpha_{1}$ is the order of $J$ along the first center of transformation. Hence $\alpha_{1} / b=\operatorname{ord}_{\mathcal{B}_{0}}\left(\xi_{0}\right)$, and then $\operatorname{ord}_{\mathcal{B}_{1}}\left(\xi_{1}\right)=\alpha_{1} / b+\mathrm{w}-\operatorname{ord}_{1}\left(\xi_{1}\right)$ and (i) holds in this case.

Assume that (i) holds for $i=k-1$, and we want to prove the assertion for $i=k$. Then we argue as above distinguishing the case when $\xi_{k} \in H_{k}$, or $\xi_{k} \notin H_{k}$. A general expression of the invariants mentioned in (i) in terms of $\left\{\operatorname{ord}_{\mathcal{B}_{0}}\left(\xi_{0}\right), \operatorname{ord}_{\mathcal{B}_{1}}\left(\xi_{1}\right), \ldots, \operatorname{ord}_{\mathcal{B}_{k}}\left(\xi_{k}\right)\right\}$ can be found in [18, Theorem 7.6].

Parts (ii) and (iii) follow from the proof of part (i).

15.25. Proof of Proposition 15.18: Let $(W,(J, b), E)$ be a basic object, and assume by induction on $k$, that we have defined an equivariant sequence of transformations

$$
\left(W_{0},\left(J_{0}, b\right), E_{0}\right) \longleftarrow \ldots \longleftarrow\left(W_{k},\left(J_{k}, b\right), E_{k}\right),
$$

at centers $C_{i} \subset \underline{\operatorname{Max}}^{\mathrm{M}^{\prime}-\mathrm{ord}_{i}}$ for $i=0,1, \ldots, k-1$. Hence, if $\Theta: \mathcal{B}=$ $(W,(J, b), E) \rightarrow \mathcal{B}^{\prime}=\left(W^{\prime},\left(J^{\prime}, b^{\prime}\right), E^{\prime}\right)$ is an isomorphism, then there is an induced sequence of transformations

$$
\mathcal{B}^{\prime}=\left(W_{0}^{\prime},\left(J_{0}^{\prime}, b^{\prime}\right), E_{0}^{\prime}\right) \longleftarrow \ldots \longleftarrow \mathcal{B}_{k}^{\prime}=\left(W_{k}^{\prime},\left(J_{k}^{\prime}, b^{\prime}\right), E_{k}^{\prime}\right)
$$

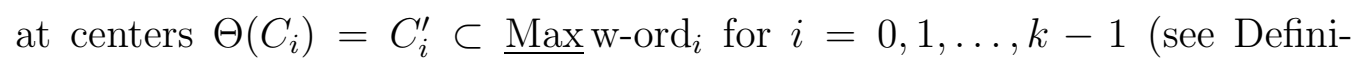
tion 4.6).

Let $k_{0}$ be the smallest index in sequence (15.25.1) (or in (15.25.2)) such that

$$
\max \mathrm{w}-\operatorname{ord}_{k_{0}}=\max \mathrm{w}-\operatorname{ord}_{k} .
$$

We distinguish two cases: 
- Case $\max \mathrm{w}-\operatorname{ord}_{k_{0}}>0$.

Recall that in this case, if we set $\max \mathrm{w}-\operatorname{ord}_{k_{0}}=b^{\prime} / b$, the basic object

$$
\left(W_{k_{0}},\left(A_{k_{0}}, c\right), E_{k_{0}}\right)=\left(W_{k_{0}},\left(\overline{J_{k_{0}}}, b^{\prime}\right), E_{k_{0}}\right) \cap\left(W_{k_{0}},\left(J_{k_{0}}, b\right), E_{k_{0}}\right)
$$

is within the previously treated Case 2 ; moreover, $\operatorname{Sing}\left(A_{k_{0}}, c\right)=\underline{\operatorname{Max}} \mathrm{w}-\operatorname{ord}_{k_{0}}$, and the basic object $\left(W_{k_{0}},\left(A_{k_{0}}, c\right), E_{k_{0}}\right)$ fulfills all conditions stated in 15.9. As indicated in Remark $15.10(\mathrm{~A})$, a resolution of $\left(W_{k_{0}},\left(A_{k_{0}}, c\right), E_{k_{0}}\right)$, say

$$
\left(W_{k_{0}},\left(A_{k_{0}}, c\right), E_{k_{0}}\right) \longleftarrow \ldots \longleftarrow\left(W_{N},\left(A_{N}, c\right), E_{N}\right),
$$

induces a sequence

$$
\left(W_{k_{0}},\left(J_{k_{0}}, b\right), E_{k_{0}}\right) \longleftarrow \ldots \longleftarrow\left(W_{N},\left(J_{N}, b\right), E_{N}\right)
$$

such that

$\max \mathrm{w}-\operatorname{ord}_{k_{0}}=\max \mathrm{w}-\operatorname{ord}_{k_{0}+1}=\cdots=\max \mathrm{w}-\operatorname{ord}_{N-1}>\max \mathrm{w}-\operatorname{ord}_{N}$.

Assume that the last $k-k_{0}$ steps of sequence (15.25.1) are the first $k-k_{0}$ steps of the sequence (15.25.4) (induced by the resolution (15.25.3)), and now extend sequence (15.25.1), as above, to a sequence of length $N$. Note that $N>k$ and that all the hypothesis on sequence (15.25.1) also hold on this enlarged sequence. In particular, if sequence (15.25.2) is defined in terms of sequence (15.25.1), then by Lemma 15.24 and the equivariance of Case 2, sequence (15.25.2) can also be extended to a sequence of length $N$, such that $\mathrm{w}_{-} \operatorname{ord}_{k_{0}}>\max \mathrm{w}-\operatorname{ord}_{N}$, and the isomorphisms $\Theta_{j}, j=0,1, \ldots, k$ can be lifted for $j=k+1, \ldots, N$.

If $\operatorname{maxw}-\operatorname{ord}_{N}>0$ we repeat the argument. But this can happen only finitely many times. In fact, if $\max \mathrm{w}-\mathrm{ord}_{k}=b^{\prime} / b$, then this positive rational number can drop at most $b^{\prime}$ times. This leads us ultimately to the case $\max \mathrm{w}$-ord $=0$.

- Case $\max \mathrm{w}-\operatorname{ord}_{k_{0}}=0$.

In this case we have that

$$
J_{k_{0}}=\mathcal{I}\left(H_{1}\right)^{\alpha(1)} \mathcal{I}\left(H_{2}\right)^{\alpha(2)} \cdot \ldots \cdot \mathcal{I}\left(H_{k_{0}}\right)^{\alpha\left(k_{0}\right)},
$$

and hence also that

$$
J_{k_{0}}^{\prime}=\mathcal{I}\left(H_{1}^{\prime}\right)^{\alpha(1)} \mathcal{I}\left(H_{2}^{\prime}\right)^{\alpha(2)} \cdot \ldots \cdot \mathcal{I}\left(H_{k_{0}}^{\prime}\right)^{\alpha\left(k_{0}\right)}
$$


This is what we call the monomial case which will be treated in Section 20 . We will only mention here that in this case it is very simple to extend sequence (15.25.1) (and hence sequence (15.25.2)) to a resolution. This can be done in various ways, and simply by considering the exponents $\alpha(i)$ : One can define a new upper semi-continuous equivariant function whose value at $\xi \in \operatorname{Sing}\left(J_{k}, b\right)$ depends entirely on $\left\{\alpha(i) / b: \xi \in H_{i} \in E_{k}\right\}$. These functions are equivariant by Lemma 15.24 (ii), and reach their maximum value at a smooth permissible center. An extension of the first $k_{0}$ steps of the equivariant sequence (15.25.1) (of (15.25.2)) to an equivariant resolution is achieved by repeating this procedure (see Section 20 ).

\section{Proof of Theorem 14.8 for general basic objects}

We now address the proof of Theorem 14.8 for general basic objects $\mathcal{B}=$ $(\mathcal{F},(W, E))$ of dimension $d$. Fix an open covering $\left\{U_{\alpha}\right\}_{\alpha \in \Lambda}$, a collection of smooth $d$-dimensional subschemes $\widetilde{W}_{\alpha} \subset U_{\alpha}$ and $\mathcal{B}_{\alpha}=\left(\widetilde{W}_{\alpha},\left(B_{\alpha}, d_{\alpha}\right) \widetilde{E}_{\alpha}\right)$ with the properties stated in Definition 14.1. Recall that by Proposition 14.7, there are well defined equivariant functions,

$$
\operatorname{ord}^{d}: F \rightarrow \mathbb{Q} \text { and } n^{d}: F \rightarrow \mathbb{Z},
$$

obtained by patching the functions $\operatorname{ord}_{\mathcal{B}_{\alpha}}^{d}$ and $n_{\mathcal{B}_{\alpha}}^{d}$.

As in the case of basic objects we will consider three cases:

- Case 1: $\operatorname{ord}_{\mathcal{B}}=1$ and $E=\emptyset$.

- Case 2: $\operatorname{ord}_{\mathcal{B}}=1$ and $E \neq \emptyset$.

- Case 3. The general case: Proof of Theorem 14.8.

Again, we stress here that Cases 1 and 2 are not special cases, and that they appear as intermediate steps when proving the general case. Recall that Theorem 14.8 holds for $d=0$, so we will argue by induction on $d$.

\section{- Case 1.}

Since $\mathcal{F}$ is of dimension $d$, the closed set $F(\subset W)$, has dimension at most $d-1$. Lemma 15.4 asserts that the $(d-1)$-dimensional components of $F$ are both open and closed in $F$, defining a smooth permissible center; and furthermore, by blowing up such center the transform has a structure of $(d-1)$ dimensional general basic object. A similar argument as the one used in the proof of Proposition 15.1 shows that this particular transformation is equivariant. This proves the theorem for $d$-dimensional general basic objects within Case 1. 
Remark 16.1. We may also repeat the outcome of Remark 15.8 in the context of general basic objects, namely that the construction of the resolution indicated above, also applies for transform of a general basic object within Case 1.

\section{- Case 2.}

Let $\mathcal{F}_{0}$ be a general basic object within Case 2. As in Proposition 14.7, set

$$
n_{\mathcal{F}_{0}}: F_{0} \rightarrow \mathbb{N}
$$

Given a sequence of transformation of general basic objects,

$$
\left(\mathcal{F}_{0},\left(W_{0}, E_{0}\right)\right) \longleftarrow \ldots \longleftarrow\left(\mathcal{F}_{r-1},\left(W_{r-1}, E_{r-1}\right)\right) \longleftarrow\left(\mathcal{F}_{r},\left(W_{r}, E_{r}\right)\right) .
$$

we can consider the partition, as in (15.13.2),

$$
E_{k}=E_{k}^{+} \cup E_{k}^{-}
$$

Finally define the functions

$$
\begin{gathered}
n_{k}: F_{k} \rightarrow \mathbb{Z} \\
n_{k}(x)=\sharp\left\{H_{i} \in E_{k}^{-} \quad: x \in H_{i}\right\} .
\end{gathered}
$$

In this way we extend, to general basic objects, the previously defined function on basic objects. The same holds for the $\mathcal{P}_{k}(m)$ in (15.14.1) as we sketch below. For each $\alpha \in \Lambda$ (as in the covering introduced in 14.1), define basic objects

$$
\left(\widetilde{W}_{\alpha, k},\left(A_{\alpha, k}, c_{\alpha}\right), \widetilde{E}_{\alpha, k}^{+}\right) \quad \text { and } \quad\left(\widetilde{W}_{\alpha, k},\left(A_{\alpha, k}, c_{\alpha}\right), \widetilde{E}_{\alpha, k}\right),
$$

with

$$
\left(\widetilde{W}_{\alpha, k},\left(A_{\alpha, k}, c_{\alpha}\right), \widetilde{E}_{\alpha, k}\right) \subset\left(\widetilde{W}_{\alpha, k},\left(B_{\alpha, k}, d_{\alpha}\right), \widetilde{E}_{\alpha, k}\right)
$$

as in (15.14.2) and (15.14.3):

$$
\left(\widetilde{W}_{\alpha, k},\left(A_{\alpha, k}, c_{\alpha}\right), \widetilde{E}_{\alpha, k}^{+}\right)=\left(\widetilde{W}_{\alpha, k},\left(B_{\alpha, k}, d_{\alpha}\right), \widetilde{E}_{\alpha, k}^{+}\right) \cap\left(\widetilde{W}_{\alpha, k},\left(\mathcal{P}_{\alpha, k}, 1\right), E_{k}^{+}\right),
$$

and

$$
\left(\widetilde{W}_{\alpha, k},\left(A_{\alpha, k}, c\right) \widetilde{E}_{\alpha, k}\right)=\left(\widetilde{W}_{\alpha, k},\left(B_{\alpha, k}, d\right) \widetilde{E}_{\alpha, k}\right) \cap\left(\widetilde{W}_{\alpha, k},\left(\mathcal{P}_{\alpha, k}, 1\right), E_{k}\right) .
$$


Now note that the $\left(\widetilde{W}_{\alpha, k},\left(A_{\alpha, k}, c\right) \widetilde{E}_{\alpha, k}\right)$ define a new $d$-dimensional general basic objects (see Remark $15.10(\mathrm{C})$ ). So for $k=0$ we obtain a new general basic object, say $\left(\mathcal{F}_{0}^{\prime},\left(W_{0}, E_{0}\right)\right)$, and we repeat the argument used in Case 2 in the context of basic objects: Since $\left(\mathcal{F}_{0}^{\prime},\left(W_{0}, E_{0}\right)\right)$ is in Case 1 there is an equivariant resolution,

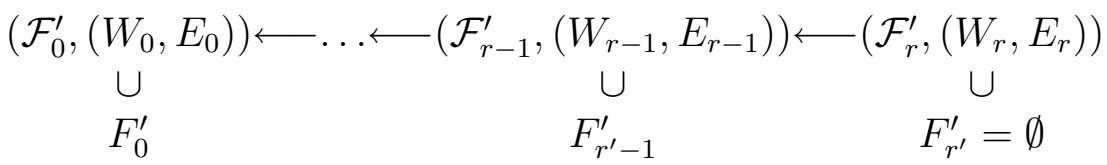

which induces a sequence of transformations

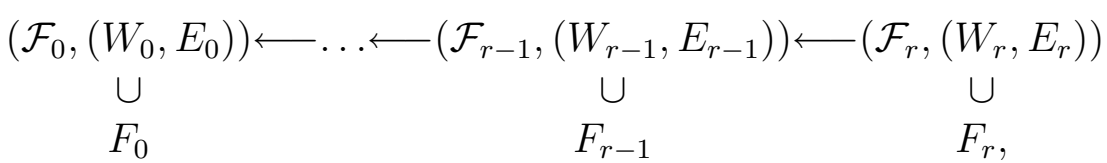

such that $\max n_{0}=\max n_{1}=\cdots=\max n_{r-1}>\max n_{r}$, and $F_{i}^{\prime}=\underline{\operatorname{Max}} n_{i} \subset$ $F_{i}$ for $i=0,1, \ldots, r-1$ (see Remark $15.10(\mathrm{~A})$ ).

If $\max n_{r} \neq 0$, a new $d$-dimensional general basic object is attached to the value $\max n_{r}$, as we did before. We repeat this argument up to the case in which $\max n_{r}=0$. In this case $\left(\mathcal{F}_{r},\left(W_{r}, E_{r}\right)\right)$ is within Case 1 (in the sense of Remark 16.1), and hence can be extended to a resolution.

Assume now that there is an isomorphism of general basic objects, say $\mathcal{F} \rightarrow \mathcal{G}$. If $\mathcal{F}^{\prime}$ and $\mathcal{G}^{\prime}$ are both defined, as before, in terms of the value $\max n$, then by Remark $15.10(\mathrm{~B}), \mathcal{F}^{\prime} \cong \mathcal{G}^{\prime}$ (see also Remark $15.10(\mathrm{D})$ ). Hence these resolutions are equivariant.

\section{- Case 3: Proof of Theorem 14.8}

We may argue as in the previous case, to show that the functions w-ord $\mathrm{d}_{i}$ can also be defined for general basic objects, and for a sequence of transformations of general basic objects. The same arguments used above, replacing here the functions $n_{i}$ by w-ord ${ }_{i}$, show that:

(i) For any general basic object $\mathcal{F}_{0}$, we can associate to it a sequence of transformations,

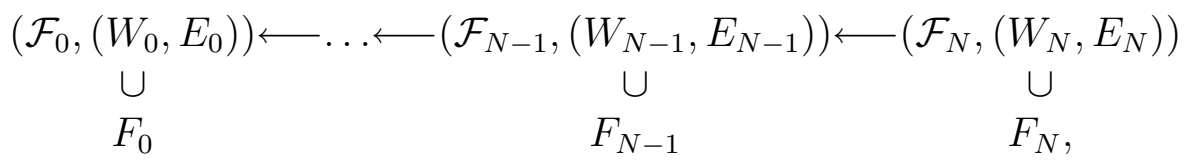

such that $\max \mathrm{w}-\operatorname{ord}_{N}=0$.

(ii) Given an isomorphism $\Theta: \mathcal{F}_{0} \rightarrow \mathcal{F}_{0}^{\prime}$, then the sequences defined in (i) for $\mathcal{F}_{0}$ and $\mathcal{F}_{0}^{\prime}$, are both of same length, say $N$, and linked by isomorphisms $\Theta_{i}: \mathcal{F}_{i} \rightarrow \mathcal{F}_{i}^{\prime}$ for $0 \leq i \leq N$. 
Finally, the discussion in Case w-ord ${ }_{N}=0$ applies also for general basic objects. In fact the extension of sequence (16.1.2) to a resolution is achieved by means of a function that depends only on the invariants of Lemma 15.24. Thus, Lemma 15.24 together with the equivariance of the functions $\operatorname{ord}_{\mathcal{F}_{i}}$ assert that the extensions of sequence (16.1.2) to a resolution of $\mathcal{F}_{0}$ (of $\mathcal{F}_{0}^{\prime}$ ) are defined together with linking isomorphisms. This proves Theorem 14.8.

\section{Part V. The Algorithm}

\section{On the definition of the functions $f_{i}^{d}$}

We address here the explicit description of the functions $f_{i}^{d}$ introduced in Definition 5.5. These functions provide the elementary proofs of desingularization and principalization given in 5.8 and 5.9; and the resolution of basic objects they define turn out being that defined in Theorem 14.8. We summarize some previous results for self-containment, although some references to Part IV will be needed.

17.1. Let $\left(W_{0},\left(J_{0}, b\right), E_{0}=\left\{H_{1}, \ldots, H_{l}\right\}\right)$ be a $d$-dimensional basic object. Given a sequence of transformations,

$$
\left(W_{0},\left(J_{0}, b\right), E_{0}\right) \stackrel{\pi_{1}}{\longleftarrow} \ldots \stackrel{\pi_{r}}{\longleftarrow}\left(W_{r},\left(J_{r}, b\right), E_{r}\right)
$$

we define expressions

$$
J_{i}=\mathcal{I}\left(H_{l+1}\right)^{a_{1}} \ldots \mathcal{I}\left(H_{l+i}\right)^{a_{r}} \bar{J}_{i}
$$

where $H_{l+i}$ denotes the exceptional divisor at that $i$-th blowing-up, and upper semi-continuous functions

$$
\begin{array}{ccc}
\mathrm{w}_{-} \operatorname{ord}_{i}^{d}: \operatorname{Sing}\left(J_{i}, b\right) & \longrightarrow \frac{1}{b} \mathbb{Z} \subset \mathbb{Q} \\
\xi & \longrightarrow \frac{\nu_{\bar{J}_{i}}(\xi)}{b}
\end{array}
$$

are defined in terms of (17.1.2) (see (15.20.2) and Definition 15.22).

Assume now that sequence (17.1.1) is a sequence of transformations at permissible centers $Y_{i}$ such that

$$
Y_{i} \subset \underline{\operatorname{Max}} \mathrm{w}-\operatorname{ord}_{i}^{d} \subset \operatorname{Sing}\left(J_{i}, b\right)
$$

for $i=0,1, \ldots, r$. In this case:

$$
\mathrm{w}-\operatorname{ord}_{i-1}^{d}\left(\pi_{i}\left(\xi_{i}\right)\right) \geq \mathrm{w}-\operatorname{ord}_{i}^{d}\left(\xi_{i}\right)
$$

for every $\xi_{i} \in \operatorname{Sing}\left(J_{i}, b\right)$, and equality holds if $\pi_{i}\left(\xi_{i}\right) \notin Y_{i-1}$ (see Remark 13.16). 
In particular

$$
\max \mathrm{w}-\operatorname{ord}_{0}^{d} \geq \cdots \geq \max \mathrm{w}-\operatorname{ord}_{r}^{d}
$$

Pick $k \in\{0,1, \ldots, r\}$. If $\max _{\mathrm{w}-\operatorname{ord}_{k}^{d}}^{d}>0$ let $k_{0}$ be the smallest index so that

$$
\operatorname{max~w-ord} k_{k_{0}-1}^{d}>\operatorname{maxw}-\operatorname{ord}_{k_{0}}^{d}=\operatorname{max~w-ord}_{k}^{d},
$$

$\left(k_{0}=0\right.$ if $\left.\max \mathrm{w}-\operatorname{ord}_{0}^{d}=\cdots=\operatorname{max~w-ord}_{r}^{d}\right)$. Write

$$
E_{k}=E_{k}^{+} \sqcup E_{k}^{-}
$$

where $E_{k}^{-}$is the set hypersurfaces of $E_{k}$ which are strict transforms of hypersurfaces of $E_{k_{0}}$. Now define

$$
n_{k}^{d}(\xi)=\left\{\begin{array}{l}
\#\left\{H \in E_{k} \mid \xi \in H\right\} \quad \text { if } \quad \mathrm{w}-\operatorname{ord}_{k}^{d}(\xi)<\operatorname{maxw}_{-} \operatorname{ord}_{k}^{d} \\
\#\left\{H \in E_{k}^{-} \mid \xi \in H\right\} \quad \text { if } \quad \mathrm{w}-\operatorname{ord}_{k}^{d}(\xi)=\operatorname{maxw}_{-} \operatorname{ord}_{k}^{d}
\end{array}\right.
$$

Definition 17.2. If condition (17.1.3) holds, and $\max \mathrm{w}-\mathrm{ord}_{r}^{d}>0$, we define, for the index $r$, a function $t_{r}^{d}$ by setting:

$$
\begin{array}{ccc}
t_{r}^{d}: \operatorname{Sing}\left(J_{r}, b\right) & \longrightarrow & (\mathbb{Q} \times \mathbb{Z}, \leq) \\
\xi & \longrightarrow & \left(\operatorname{w-ord}_{r}^{d}(\xi), n_{r}^{d}(\xi)\right),
\end{array}
$$

where $\mathbb{Q} \times \mathbb{Z}$ is ordered lexicographically. In the same way we define functions $t_{r-1}^{d}, t_{r-2}^{d}, \ldots, t_{0}^{d}$. If $Y_{r} \subset \underline{\operatorname{Max}} t_{r}^{d}$ is a permissible center, then $Y_{r}$ is said to be a $t_{r}^{d}$-permissible center.

17.3. Properties of the inductive function $t_{i}^{d}$ (cf. [18, 4.15]).

1. Set $F^{i}=\operatorname{Sing}\left(J_{i}, b\right)$. Given $(q, m) \in \mathbb{Q} \times \mathbb{Z}$, set

$$
F_{(q, m)}^{i}=\left\{x \in F^{i}: t_{i}^{d}(x) \geq(q, m)\right\} .
$$

Note that

$$
F_{(q, m)}=\left\{x \in F^{i}: \mathrm{w}_{-} \operatorname{ord}_{i}(x)>q\right\} \cup\left\{x \in F^{i}: \mathrm{w}-o r d_{i}(x) \geq q, n_{i}(x) \geq m\right\} .
$$

Here the first term is closed since w-ord is upper semi-continuous and takes only finitely many different values. Since $n_{i}$ and w-ord ${ }_{i}$ are upper semi-continuous functions, it easily follows that the second term is also closed. This shows that $F_{(q, m)}$ is closed, and hence that each $t_{i}^{d}$ is upper

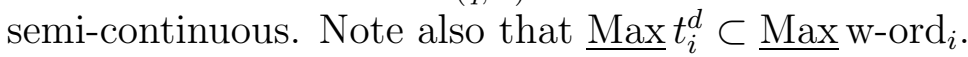


2. If the sequence of transformations

$$
\left(W_{0},\left(J_{0}, b\right), E_{0}\right) \stackrel{\pi_{1}}{\longleftarrow}\left(W_{1},\left(J_{1}, b\right), E_{1}\right) \stackrel{\pi_{2}}{\longleftarrow} \ldots \stackrel{\pi_{r}}{\longleftarrow}\left(W_{r},\left(J_{r}, b\right), E_{r}\right)
$$

is $t^{d}$-permissible, namely if $Y_{i} \subset \underline{\operatorname{Max}} t_{i}^{d}(\subset \underline{\text { Max } \text {-ord }} i)$, then for each index $i=0,1, \ldots, r$,

$$
t_{i-1}^{d}\left(\pi_{i}\left(\xi_{i}\right)\right) \geq t_{i}^{d}\left(\xi_{i}\right)
$$

for all $\xi_{i} \in \operatorname{Sing}\left(J_{i}, b\right)$, and equality holds if $\pi_{i}\left(\xi_{i}\right) \notin Y_{i-1}$. In particular

$$
\max t_{0}^{d} \geq \cdots \geq \max t_{r}^{d}
$$

3. We say that $\max t^{d}$ drops at $i_{0}$ if $\max t_{i_{0}-1}^{d}>\max t_{i_{0}}$. If $\max \mathrm{w}-\mathrm{ord}_{0}^{d}=$ $b^{\prime} / b$ and $\operatorname{dim} W_{0}=d$, note that $\max t_{i}^{d}=(s / b, m), 0 \leq s \leq b^{\prime}, 0 \leq$ $m \leq d$. So it is clear that $\max t^{d}$ can drop at most $b^{\prime} d$ times.

4. The functions $t_{i}^{d}$ are the inductive invariants. In fact, if

$$
R(1)(\underline{\operatorname{Max}} t) \neq \emptyset
$$

(see 13.1), then this is our canonical choice of center; and after repeatedly blowing up this center we may assume that $R(1)(\underline{\operatorname{Max}} t)=\emptyset$. On the other hand, if $R(1)(\underline{\operatorname{Max}} t)=\emptyset$, then via some form of induction which will be described below, it is possible to construct a unique enlargement of sequence (17.3.1),

$$
\begin{aligned}
& \left(W_{0},\left(J_{0}, b\right), E_{0}\right) \longleftarrow \ldots \longleftarrow\left(W_{r},\left(J_{r}, b\right), E_{r}\right) \longleftarrow \\
& \longleftarrow\left(W_{r+1},\left(J_{r+1}, b\right), E_{r+1}\right) \longleftarrow \ldots \longleftarrow\left(W_{N},\left(J_{N}, b\right), E_{N}\right),
\end{aligned}
$$

such that $\max t_{r}^{d}=\max t_{r+1}^{d}=\cdots=\max t_{N-1}^{d}$ and either

(a) $\operatorname{Sing}\left(J_{N}, b\right)=\emptyset$; or

(b) $\operatorname{Sing}\left(J_{N}, b\right) \neq \emptyset$; and $\max \mathrm{w}-\operatorname{ord}_{N}=0$; or

(c) $\operatorname{Sing}\left(J_{N}, b\right) \neq \emptyset, \max \mathrm{w}-\operatorname{ord}_{N}>0$ and $\max t_{N-1}>\max t_{N}$.

Note that Property 3 says that the function max w-ord ${ }^{d}$ can drop at most finitely many times, in particular for some index $N$, either (a) or (b) will hold. We show now why Property (4) holds, and why the construction of the equivariant resolution $R_{\mathcal{F}}$, in Theorem 14.8, follows essentially from the function $t_{j}^{i}$ : Roughly speaking, we attach a new basic object to $\max t_{r}^{d}$, say 
$\left(W_{r},\left(J_{r}^{\prime \prime}, b^{\prime \prime}\right), E_{r}\right)$, so that $\underline{\operatorname{Max}} t_{r}^{d}=\operatorname{Sing}\left(J_{r}^{\prime \prime}, b^{\prime \prime}\right)$, and a sequence of transformations

$$
\left(W_{r},\left(J_{r}^{\prime \prime}, b^{\prime \prime}\right), E_{r}\right) \longleftarrow \ldots \longleftarrow\left(W_{N},\left(J_{N}^{\prime \prime}, b^{\prime \prime}\right), E_{N}\right)
$$

is naturally associated to sequence (17.3.2), so that $\underline{\operatorname{Max}} t_{i}^{d}=\operatorname{Sing}\left(J_{r}^{\prime \prime}, b^{\prime \prime}\right)$ $(i=r, r+1, \ldots, N-1)$, and such that $\max t^{d}{ }_{N}<\max t^{d}{ }_{N-1}$ if and only if $\operatorname{Sing}\left(J_{N}^{\prime \prime}, b^{\prime \prime}\right)=\emptyset$ (if and only if sequence (17.3.3) is a resolution). The point is that a resolution of $\left(W_{r},\left(J_{r}^{\prime \prime}, b^{\prime \prime}\right), E_{r}\right)$ is easy to achieve by induction, essentially as in 13.3, which we prove in 19. We will do this in two steps, by first attaching a basic object to the function $\mathrm{w}-\mathrm{ord}^{d}$ (in the sense described below, see 15.9 for a more precise description), and finally to the function $t^{d}$.

17.4. Fix a $d$-dimensional basic objects $\left(W_{0},\left(J_{0}, b\right), E_{0}\right)$, and let

$$
\left(W_{0},\left(J_{0}, b\right), E_{0}\right) \longleftarrow \ldots \longleftarrow\left(W_{r},\left(J_{r}, b\right), E_{r}\right)
$$

be a $t_{i}^{d}$-permissible sequence (so $\max \mathrm{w}-\operatorname{ord}_{i}^{d} \geq \operatorname{maxw}-\operatorname{ord}_{i+1}^{d}$ and $\max t_{i}^{d} \geq$ $\left.\max t_{i+1}^{d}\right)$. For each $i=0,1, \ldots, r$, consider the factorization

$$
J_{i}=\mathcal{I}\left(H_{l+1}\right)^{a_{1}} \cdot \ldots \cdot \mathcal{I}\left(H_{l+i}\right)^{a_{i}} \bar{J}_{i}
$$

as in (17.1.2). We will assume that sequence (17.4.1) has been defined by induction on $r$, together with some other added conditions that we will impose below.

- Case $\max \mathrm{w}-\operatorname{ord}_{r}>0$.

The function $t_{r}^{d}$ was defined only when $\operatorname{maxw}-\operatorname{ord}_{r}^{d}>0$, and in that case

$$
\underline{\operatorname{Max}} t_{i}^{d} \subset \underline{\operatorname{Max}} \mathrm{w}-\operatorname{ord}_{i}^{d} \subset V\left(\bar{J}_{i}\right) .
$$

Set $r_{0}(\leq r)$ the smallest index such that $\max \mathrm{w}-\operatorname{ord}_{r_{0}}=\operatorname{maxw}_{\mathrm{w}} \operatorname{ord}_{r}$. A basic object

$$
\left(W_{r_{0}},\left(J_{r_{0}}^{\prime}, b^{\prime}\right), E_{r_{0}}\right)
$$

can be attached to the value max w-ord $\mathrm{r}_{0}$, in the sense indicated above (and denoted by $\left(W_{r_{0}},\left(A_{r_{0}}, c\right), E_{r_{0}}\right)$ in $(15.23 .1)$, see Remark $\left.15.23(\mathrm{e})\right)$. Here all the centers $Y_{i} \subset$ Max w-ord $_{i}$, and we will assume that the last $r-r_{0}$ steps of (17.4.1) are defined by a sequence of transformations

$$
\left(W_{r_{0}},\left(J_{r_{0}}^{\prime}, b^{\prime}\right), E_{r_{0}}\right) \leftarrow\left(W_{r_{0}+1},\left(J_{r_{0}+1}^{\prime}, b^{\prime}\right), E_{r_{0}+1}\right) \leftarrow \ldots \leftarrow\left(W_{r},\left(J_{r}^{\prime}, b^{\prime}\right), E_{r}\right)
$$

as in the case of sequence (17.3.3) (or see (P1) in 15.9). 
Note that $\left(W_{r_{0}},\left(J_{r_{0}}^{\prime}, b^{\prime}\right), E_{r_{0}}\right)$ is closer to the setting in 13.3 , in fact ord $\cong 1$ (it is within Case 2, see Remark $15.23(\mathrm{~d})$ ). Consider the partition $E_{j}=E_{j}^{+} \cup E_{j}^{-}$, for each index $r_{0} \leq j \leq r$, and assume that sequence (17.4.2) is such that

$$
\max n_{r_{0}} \geq \max n_{r_{0}+1} \geq \cdots \geq \max n_{r} .
$$

Since $n_{j}$ is the second coordinate of $t_{j}$ we just view it as a function on $\underline{\operatorname{Max}} \mathrm{w}_{-} \operatorname{ord}_{j}=\operatorname{Sing}\left(J_{j}^{\prime}, b^{\prime}\right)$. Set $r_{1}\left(\geq r_{0}\right)$ the smallest index such that $\max n_{r_{1}}=$ $\max n_{r}$ and let

$$
\left(W_{r_{1}},\left(J_{r_{1}}^{\prime \prime}, b^{\prime \prime}\right), E_{r_{1}}\right)
$$

be the basic object attached to $\max n_{r_{1}}$ in (15.14.3) (see also Remark 15.15(b)).

All centers $Y_{i} \subset \underline{\operatorname{Max}} n_{i}\left(=\underline{\operatorname{Max}} t_{i} \subset \underline{\operatorname{Max}} \mathbf{w}-\operatorname{ord}_{i}\right)$, and we assume that the last $r-r_{1}$ terms of sequence (17.4.2) are defined by

$$
\left(W_{r_{1}},\left(J_{r_{1}}^{\prime \prime}, b^{\prime \prime}\right), E_{r_{1}}\right) \leftarrow\left(W_{r_{1}+1},\left(J_{r_{1}+1}^{\prime \prime}, b^{\prime \prime}\right), E_{r_{1}+1}\right) \leftarrow \cdots \leftarrow\left(W_{r},\left(J_{r}^{\prime \prime}, b^{\prime \prime}\right), E_{r}\right)
$$

as in the case of 17.3 .3 (or see (P1) in 15.9).

The point is that $\left(W_{r_{1}},\left(J_{r_{1}}^{\prime \prime}, b^{\prime \prime}\right), E_{r_{1}}\right) \cong\left(W_{r_{1}},\left(J_{r_{1}}^{\prime \prime}, b^{\prime \prime}\right), E_{r_{1}}^{+}\right)$(see $\left.(15.15 .2)\right)$, and the right hand term is essentially in the setting of 13.3 (it is within Case 1, see (15.17)). It follows now from 15.8 that the set $F=\operatorname{Sing}\left(J^{\prime \prime}, b^{\prime \prime}\right)=$ Max $t^{d}$ is a disjoint union $R(1)(F) \cup F_{1}$, where $R(1)(F)$ is a permissible center, and after blowing up that smooth hypersurface we may assume that $R(1)(F)=\emptyset$, in which case $\left(W_{r_{1}},\left(J_{r_{1}}^{\prime \prime}, b^{\prime \prime}\right), E_{r_{1}}\right)$ has a $(d-1)$-dimensional structure. Theorem 14.8 defines a resolution, which in this case has first center $R(1)(F) \neq \emptyset$, and then proceeds by induction on $d-1$.

Assume, by induction, that $\left(I_{d-1}, \geq\right)$ is defined, together with functions $f_{i}^{d-1}$ which have the properties stated in Definition 5.5 for general basic objects of dimension $d-1$. Assume also that the resolution that they provide, is the same resolution as Theorem (d-1) (Theorem 14.8). We also assume that $I_{d-1}$ has a biggest element, say $\infty_{d-1} \in I_{d-1}$, and that this value is never reached by any $f_{i}^{d-1}$.

Set $\bar{f}_{r_{1}}^{d-1}(x)=\infty_{d-1}$ if $x \in R(1)(F)$, and $\bar{f}_{r_{1}}^{d-1}(x)=f_{0}^{d-1}(x)$ if $x \in F_{1}$. So if $R(1)(F) \neq \emptyset$ the following properties hold:

$$
\begin{aligned}
& \underline{\operatorname{Max}} \bar{f}_{r_{1}}^{d-1}=R(1)(F) ; \quad \max \bar{f}_{r_{1}}^{d-1}>\max \bar{f}_{r_{1}+1}^{d-1} \\
& \bar{f}_{r_{1}}^{d-1}(x)=\bar{f}_{r_{1}+1}^{d-1}\left(x^{\prime}\right) \quad \text { if } \quad x \notin R(1)(F),
\end{aligned}
$$

where $x^{\prime} \in \operatorname{Sing}\left(J_{r_{1}+1}^{\prime \prime}, b^{\prime \prime}\right)$ is, in this case, the point naturally identified with $x$, and assume that the first transformation in sequence (17.4.3) is defined by blowing up at $\underline{\operatorname{Max}} \bar{f}_{i}^{d-1}=R(1)(F)$ (see Case 1 in 15.7). 
Fix some index $i, r_{1}<i \leq r$. For any

$$
x \in \underline{\operatorname{Max}} n_{i} \quad\left(=\underline{\operatorname{Max}} t_{i} \subset \underline{\operatorname{Max}} \mathrm{w}-\operatorname{ord}_{i}\right)
$$

set

$$
\bar{f}_{i}^{d-1}(x)=f_{i-r_{1}}^{d-1}(x)
$$

and assume that sequence (17.4.3) is defined by blowing up at $\underline{\operatorname{Max}} \bar{f}_{i}^{d-1}$. Extend sequence (17.4.3) to a resolution by means of these functions. Finally extend sequence (17.4.1) by this resolution in the sense of 15.9 (P1). Since $\max t^{d}$ can drop only finitely many times, eventually we come to the following case.

- Case max w-ord ${ }_{r}=0$.

Set $r_{0} \leq r$ the smallest index for which $\operatorname{maxw}-\operatorname{ord}_{r_{0}}=0$. This is the case in which $J_{r_{0}}$ is in the setting of Remark 15.23 (b). This is a simple case, in which no form of induction is required. It is not hard to formulate the development in the proof of Case w-ord $=0$ in Proposition 15.18, by defining a totally ordered set $(\Gamma, \geq)$ and an upper semi-continuous function $h_{r_{0}}: \operatorname{Sing}\left(J_{r_{0}}, b\right) \rightarrow \Gamma$ so that, as in that proof, $\underline{\operatorname{Max}} h_{r_{0}}$ is a permissible center (see Section 20). Furthermore, by setting inductively functions $h_{i}$ : $\operatorname{Sing}\left(J_{i}, b\right) \rightarrow \Gamma$, a resolution

$$
\left(W_{r_{0}},\left(J_{r_{0}}, b\right), E_{r_{0}}\right) \longleftarrow \ldots \longleftarrow\left(W_{N},\left(J_{N}, b\right), E_{N}\right)
$$

is defined by blowing up at $\left.\underline{\operatorname{Max}} h_{i} \subset \operatorname{Sing}\left(J_{i}, b\right), E_{i}\right)$ for $i=0,1, \ldots, N-1$, and the sequence is such that

$$
\max h_{j}>\max h_{j+1} ; \quad h_{j}^{d}(x)=h_{j+1}^{d}\left(x^{\prime}\right) \text { if } x \notin \underline{\operatorname{Max}} h_{j},
$$

where $x^{\prime} \in \operatorname{Sing}\left(J_{j+1}, b\right)$ is, in this case, the point naturally identified with $x$. Assume that the last $r-r_{0}$ steps of sequence (17.4.1) are the first $r-r_{0}$ steps of sequence (17.4.6). Finally extend sequence (17.4.1) to a resolution of length $\mathrm{N}$.

Definition 17.5. Set $T^{d}=\{\infty\} \sqcup(\mathbb{Q} \times \mathbb{Z}) \sqcup \Gamma$ where this disjoint union is totally ordered by setting that $\infty$ is the biggest element, and that $\alpha<\beta$ if $\beta \in(\mathbb{Q} \times \mathbb{Z})$ and $\alpha \in \Gamma$. We now set $I_{d}=T^{d} \times I_{d-1}$ ordered lexicographically, and define $f_{r}^{d}: \operatorname{Sing}\left(J_{r}, b\right) \rightarrow I_{d}$.

(i) If $\max \mathrm{w}-o r d_{r}>0$, and $x \in \underline{\operatorname{Max}} t_{r}\left(\subset \operatorname{Sing}\left(J_{r}, b\right)\right): f_{r}^{d}(x)=\left(\max t_{r}, \bar{f}_{r}^{d}(x)\right)$.

(ii) If $\max \mathrm{w}-\mathrm{ord}_{r}=0: f_{r}^{d}(x)=\left(h_{r-r_{0}}(x), \infty_{d-1}\right)$. 
(iii) If $\max \mathrm{w}-\operatorname{ord}_{r}>0, x \notin \underline{\operatorname{Max}} t_{r}^{d}$ and $\mathrm{w}-\operatorname{ord}_{r}(x)>0$ : There is a smallest index $r^{\prime}>r$ in the resolution, such that $\max t_{r^{\prime}}^{d}=t_{r}^{d}(x)$. Note that $x$ can be identified with a point $x^{\prime} \in \underline{\operatorname{Max}} t_{r^{\prime}}^{d}$. Set $f_{r}^{d}(x)=f_{r^{\prime}}^{d}\left(x^{\prime}\right)$.

(iv) If $\max \mathrm{w}-\operatorname{ord}_{r}>0$ and $\mathrm{w}-\operatorname{ord}_{r}(x)=0$ : There is a smallest index $r^{\prime}>r$ in the resolution, such that $\operatorname{maxw}-\operatorname{ord}_{r^{\prime}}=0$. Note that $x$ can be identified with a point $x^{\prime} \in \operatorname{Sing}\left(J_{r^{\prime}}, b\right)$ and we set $f_{r}^{d}(x)=f_{r^{\prime}}^{d}\left(x^{\prime}\right)$.

\section{Remark 17.6. On Definition 5.5.}

(1) The value $\left(\infty, \infty_{d-1}\right) \in I_{d}$ is never reached, and up to induction on the dimension $d$, choices of centers (namely, $\operatorname{Max} f^{d}$ ) are defined either as $R(1)\left(\underline{\operatorname{Max}} t^{d}\right)$ in case $\mathrm{w}$-ord ${ }^{d}>0$, or as $\underline{\operatorname{Max}} h^{d}$ in case $\mathrm{w}$-ord ${ }^{d}=0$. This shows that the centers $\underline{\operatorname{Max}} f^{d}$ are regular and permissible.

(2) Fix, as before, a basic object $\mathcal{B}=(W,(J, b), E)$ of dimension $d$, and let $R_{\mathcal{B}}:(W,(J, b), E) \longleftarrow\left(W_{1},\left(J_{1}, b\right), E_{1}\right) \longleftarrow \ldots \longleftarrow\left(W_{M},\left(J_{M}, b\right), E_{M}\right)$

be the the resolution defined by Theorem 14.8. It follows from (17.4.4) and (17.4.7) that $\max f_{j}^{d}>\max f_{j+1}^{d}$ and that $f_{j}^{d}(x)=f_{j+1}^{d}\left(x^{\prime}\right)$ if $x \notin \underline{\operatorname{Max}} f_{j}$, where $x^{\prime} \in \operatorname{Sing}\left(J_{j+1}, b\right)$ is the point naturally identified with $x$.

(3) One can check from (2) that given $\alpha \in I_{d}$,

$$
F_{\alpha}=\left\{x \in \operatorname{Sing}\left(J_{r}, b\right): f_{r}^{d}(x) \geq \alpha\right\}=\cup \pi_{r}^{s}\left(\underline{\operatorname{Max}} f_{s}^{d}\right),
$$

where the union is taken over each index $s \geq r$ such that $\max f_{s}^{d} \geq \alpha$, and where $\pi_{r}^{s}: W_{s} \rightarrow W_{r}$ is the composite morphism. In particular $F_{\alpha}$ is closed, so the functions $f_{r}^{d}$ are upper semi-continuous.

(4) Conditions B (e) and B (f) of Definition 5.5 hold for the functions $f_{r}^{d}$. In fact, by Lemma 6.7 and Lemma 15.24, it follows that both conditions hold for the functions $h_{i}$ and $t_{i}^{d}$ since they hold for the functions ord and $n$ (see also Proposition 14.7).

(5) Note that $I_{d}=T^{d} \times \ldots \times T^{0}$, and that $(\infty, \infty, \ldots, \infty) \in I_{d}$ is the biggest element.

(6) It follows now that these functions have the properties described in Definition 5.5; and since all invariants involved rely on the functions ord and $n$ ), Definition 5.5 also applies in the setting of general basic objects. 
Example 17.7. Fix $W=\mathbb{A}_{k}^{3}, E=\emptyset, X \subset W$ a smooth subscheme and set $(W,(\mathcal{I}(X), 1), \emptyset)$. Note that $\operatorname{Sing}(\mathcal{I}(X), 1)=X$ and that $t^{3}(x)=(1,0)$ for any $x \in X$.

If $X$ is a smooth hypersurface, then $X=R(1)\left(\underline{\operatorname{Max}}\left(t^{3}\right)\right)$ so $f^{3}(x)=$ $((1,0), \infty, \infty) \in I_{3}$ for any $x \in X$.

If $X$ is a smooth curve, then $R(1)\left(\underline{\operatorname{Max}}\left(t^{3}\right)\right)=\emptyset$, and $X=R(1)\left(\underline{\operatorname{Max}}\left(t^{2}\right)\right)$. In this case $f^{3}(x)=((1,0),(1,0), \infty) \in I_{3}$ for any $x \in X$.

Remark 17.8. It turns out that given a subscheme $X \subset W$, the algorithmic resolution of $(W,(\mathcal{I}(X), 1), \emptyset)$ depends, to some extent, only on $X$ (on $\left.\mathcal{O}_{W} / I(X)\right)$. In fact, if $J \subset \mathcal{O}_{W}$ and $J^{\prime} \subset \mathcal{O}_{W^{\prime}}$ are such that $\mathcal{O}_{W} / J$ and $\mathcal{O}_{W^{\prime}} / J^{\prime}$ are isomorphic, and if

$$
R_{\mathcal{B}}:(W,(J, 1), \emptyset) \longleftarrow\left(W_{1},\left(J_{1}, 1\right), E_{1}\right) \longleftarrow \ldots \longleftarrow\left(W_{M},\left(J_{M}, 1\right), E_{M}\right)
$$

and

$$
R_{\mathcal{B}^{\prime}}:\left(W^{\prime},\left(J^{\prime}, 1\right), \emptyset\right) \longleftarrow\left(W_{1}^{\prime},\left(J_{1}^{\prime}, 1\right), E_{1}^{\prime}\right) \longleftarrow \ldots \longleftarrow\left(W_{M^{\prime}}^{\prime},\left(J_{M^{\prime}}^{\prime}, 1\right), E_{M^{\prime}}\right)
$$

are the resolutions defined by Theorem 14.8, then $M=M^{\prime}$ and, for each index $i$, there is an identification of $V\left(J_{i}\right)\left(\subset W_{i}\right)$ with $V\left(J_{i}^{\prime}\right)\left(\subset W_{i}^{\prime}\right)$. To check this, note first that if $\operatorname{dim} W=d>\operatorname{dim} W^{\prime}=d^{\prime}$, then $R_{\mathcal{B}}:(W,(J, 1), \emptyset)$ has a structure of $d-d^{\prime}$ general basic object. Hence the first $d-d^{\prime}$ coordinates of $f_{i}$ are $(1,0)$ at any point of $V\left(J_{i}\right)$. Use this argument to reduce to the case in which $d=d^{\prime}$, and then check, by induction on $i$, that in that setting, there is an identification of the set $V\left(J_{i}\right)\left(\subset W_{i}\right)$ with $V\left(J_{i}^{\prime}\right)\left(\subset W_{i}^{\prime}\right)$ such that the functions $f_{i}$ and $f_{i}^{\prime}$ coincide. See Section 9 for more details.

\section{Bodnár-Schicho's program for resolution of singu- larities}

In [10], G. Bodnár and J. Schicho presented a computer program that produces resolution of basic objects. Full details can be found in [11]. Given a basic object, $(W,(J, b), E)$ the program provides its resolution,

$$
(W,(J, b), E) \longleftarrow\left(W_{1},\left(J_{1}, b\right), E_{1}\right) \longleftarrow \ldots \longleftarrow\left(W_{r},\left(J_{r}, b\right), E_{r}\right),
$$

defined in terms of functions $f_{i}^{d}$ (see Definition 5.5).

Among the various problems to solve, the first one is how to encode the variety $W$. This is not too difficult in case $W$ is of finite type over $\mathbf{k}$. They cover $W$ by open subsets $U_{\alpha}$, such that each $U_{\alpha}$ is a closed subscheme of the affine space $\mathbb{A}_{\mathbf{k}}^{m}$, for some $m$. In fact the program presents the resolution of 
the basic object as a tree of charts. By looking at the tree one can follow the affine charts of the sequence of blowing-ups, and the top level of the tree are the charts of $W_{r}$.

From Definition 17.5 we see that the functions $f_{i}^{d}$ are defined in terms of the function ord (introduced in 6.1), and $n$ (6.2) of several auxiliary basic objects. Given an ideal $J$, the function $\operatorname{ord}(J)$ is computed in terms of the operator $\Delta(13.7)$. Finally to compute $\Delta(J)$, we need partial derivatives (see 13.6); and to compute derivatives we need regular systems of parameters at every point.

A global section $f$ of $\mathcal{O}_{U_{\alpha}}$ is a restriction of a polynomial $f \in \mathbf{k}\left[X_{1}, \ldots, X_{m}\right]$ modulo the ideal defining $U_{\alpha}$. If $f_{1}, \ldots, f_{n}$ are global sections of $\mathcal{O}_{U_{\alpha}}$ such that suitable $d \times d$ minors of the jacobian matrix do not vanish at any point of $U_{\alpha}$, then these global sections give rise to regular systems of parameters, locally at any point of $U_{\alpha}$.

18.1. In the computer program given in [11] the variety $W$ is covered by open charts, say $U_{\alpha}$, where each chart is presented as follows:

- $U_{\alpha}$ is a closed smooth variety of $\mathbb{A}_{\mathbf{k}}^{m}$ for some $m$ (depending on $U_{\alpha}$ ). So that $U_{\alpha}$ is defined by polynomials, called dependencies, $D_{1}, \ldots, D_{s} \in$ $\mathbf{k}\left[X_{1}, \ldots, X_{m}\right]$.

- Polynomials $P_{1}, \ldots, P_{d} \in \mathbf{k}\left[X_{1}, \ldots, X_{m}\right]$ which give rise to regular system of parameters for every point of $U_{\alpha}$.

- A $m \times d$ matrix with entries in $\mathbf{k}\left[X_{1}, \ldots, X_{m}\right]$, such that the restriction of each coefficient to $U_{\alpha}$ is the partial derivative of $\frac{\partial X_{i}}{\partial P_{j}}$.

With this structure they are able to compute derivatives in the chart $U_{\alpha}$. In particular, given generators of an ideal $J \subset \mathcal{O}_{U_{\alpha}}$ they compute generators of $\Delta(J)$. To compute the maximal order of $J$ in the chart $U_{\alpha}$, one has to find the smallest index $b$ such that $\Delta^{b}(J)=\mathcal{O}_{U_{\alpha}}$, which is accomplished by Gröbner basis computations.

Remark 18.2. Let $U_{\alpha}$ be a chart in 18.1 and let $C_{\alpha}=\underline{\operatorname{Max}} f_{i}^{d}$ be the center defined by the algorithm described in Definition 5.5 (i.e. by the functions $f_{i}^{d}$ ).

The center $C_{\alpha} \subset U_{\alpha}$ is defined by some equations of $\mathbf{k}\left[X_{1}, \ldots, X_{m}\right]$, say $\mathcal{I}\left(C_{\alpha}\right)=\left\langle f_{1}, \ldots, f_{r}\right\rangle$. The program will arrange matters so that $\mathcal{I}\left(C_{\alpha}\right)=$ $\left\langle P_{1}, \ldots, P_{r}\right\rangle$ for some $r<d$. Using these equations one may compute the blowing-up with center $C_{\alpha},\left(U_{\alpha}\right)_{1} \longrightarrow U_{\alpha}$, and cover $\left(U_{\alpha}\right)_{1}$ by $r$ charts as in 18.1 . 
18.3. Computing $\mathcal{I}\left(C_{\alpha}\right)$. We follow the proof of Theorem 14.8 for basic objects: If the basic object is within Case 1 of Section 15, then, and as in Proposition 13.3, at every chart $U_{\alpha}$ they define a smooth hypersurface $\widetilde{U}_{\alpha} \subset U_{\alpha}$ such that

$$
\left(U_{\alpha},\left(J_{\alpha}, b\right), E_{\alpha}\right) \subset\left(U_{\alpha},\left(\mathcal{I}\left(\widetilde{U}_{\alpha}\right), 1\right), E_{\alpha}\right) .
$$

The above inclusion asserts that $C_{\alpha} \subset \widetilde{U}_{\alpha}$, and hence, if $f \in \mathbf{k}\left[X_{1}, \ldots, X_{m}\right]$ is the equation defining the hypersurface $\widetilde{U}_{\alpha}$, then $f$ is one of the equations defining $C_{\alpha}$.

Note that the hypersurface $\widetilde{U}_{\alpha}$ may not be irreducible. If

$$
R(1)(\operatorname{Sing}(J, b)) \neq \emptyset
$$

then $R(1)(\operatorname{Sing}(J, b))$ is a smooth hypersurface and a union of some connected components of $\widetilde{U}_{\alpha}$. The program of [11] covers $U_{\alpha}$ by open sets, as in 18.1, of two types:

1. Charts where $R(1)(\operatorname{Sing}(J, b))=\widetilde{U}_{\alpha}$.

2. Charts where $R(1)(\operatorname{Sing}(J, b))=\emptyset$.

For charts of the first type the center is $C_{\alpha}=\widetilde{U}_{\alpha}$.

For charts in as in $(2)$, they provide the computations of the $(d-1)$ dimensional basic object

$$
\left(\widetilde{U}_{\alpha},\left(\operatorname{Coeff}_{\widetilde{U}_{\alpha}}(J), b !\right), E_{\alpha} \cap \widetilde{U}_{\alpha}\right),
$$

which requires an expression of $\widetilde{U}_{\alpha}$ as in 18.1. If $f=P_{j}$ for some index $j$, then $\widetilde{U}_{\alpha}$ fulfills 18.1 by adding $P_{j}$ to dependencies, and we have $d-1$ parameters.

In general $f$ will not be a parameter, and the program given in [11] uses an operation called cover and exchange: it covers $U_{\alpha}$ by charts as in 18.1 such that $f=P_{j}$ for some $j$ at each new chart. Following this procedure one reduces the computation of the center for a $d$-dimensional basic object to the computation for a $(d-1)$-dimensional basic object.

Assume now that the basic object is within Case 2 or 3 of Section 15 . In these cases new basic objects were defined, always following the general pattern of Remark 15.9. In both cases the basic objects are defined in terms of intersections of other basic objects, and this is a computation (see 12.7) which the program can also handle. 


\section{Proof of Proposition 13.3}

The objective of this section is to prove Proposition 13.3, which is a key step in the inductive proof of Theorem 14.8:

Proposition 13.3 Let $\mathcal{B}=(W,(J, b), E)$ be a basic object, assume that $E=\emptyset$ and that $\operatorname{ord}_{B} \cong 1$. Then:

a) There is an open covering $\left\{U_{\alpha}\right\}_{\alpha \in \Lambda}$, and for each index $\alpha \in \Lambda$ a closed and smooth hypersurface $\widetilde{W_{\alpha}} \subset U_{\alpha}$, such that if $\left(U_{\alpha},\left(J_{\alpha}, b\right), \emptyset\right)$ is the restriction of $(W,(J, b), \emptyset)$ to $U_{\alpha}$ then

$$
\left(U_{\alpha},\left(J_{\alpha}, b\right), \emptyset\right) \subset\left(U_{\alpha},\left(\mathcal{I}\left(\widetilde{W}_{\alpha}\right), 1\right), \emptyset\right) .
$$

b) If $U_{\alpha} \cap R(1)(\operatorname{Sing}(J, b))=\emptyset$ then $\left(U_{\alpha},\left(J_{\alpha}, b\right), \emptyset\right)$ has structure of $d-1$ dimensional basic object, i.e. there is a $d-1$ basic object $(\widetilde{W},(\mathcal{A}, e), \emptyset)$ such that

$$
\left(U_{\alpha},\left(J_{\alpha}, b\right), \emptyset\right) \cong(\widetilde{W},(\mathcal{A}, e), \emptyset) .
$$

To prove this proposition we will need some auxiliary results. Some of them clearly express the role of the derivatives in our statement, while the others are related to the study of the restriction of basic objects to smooth hypersurfaces. The proof of Proposition 13.3 will be detailed in 19.8.

\section{- The role of the derivatives (an idea of J. Giraud)}

Lemma 19.1. Let $J \subset \mathcal{O}_{W}$ be an invertible sheaf of ideals and let $\delta$ be a globally defined derivation. Then

$$
(W,(J, b), E) \cap(W,(\delta(J), b-1), E) \cong(W,(J, b), E),
$$

or equivalently,

$$
(W,(\delta(J), b-1), E) \subset(W,(J, b), E) .
$$

Proof: From the properties stated in 13.7, we have that

$$
\operatorname{Sing}(J, b) \cap \operatorname{Sing}(\delta(J), b-1)=\operatorname{Sing}(J, b) .
$$

We only have to prove that this equality is stable (or preserved) after a transformation,

$$
(W,(J, b), E) \longleftarrow\left(W_{1},\left(J_{1}, b\right), E_{1}\right) .
$$

It is simple to check this when the transformations is as in 3.12. We discuss here the case when the transformation is as in 2.3 . 
Let $H$ be the exceptional divisor, and let $\mathcal{I}(H) \subset \mathcal{O}_{W_{1}}$ be the corresponding invertible sheaf of ideals. We claim that $\mathcal{I}(H) \cdot \delta$ is an invertible sheaf of derivations on $W_{1}$. To see this we argue locally: Let $\xi \in W$ be a closed point and choose a regular system of parameters $\left\{x_{1}, \ldots, x_{n}\right\} \subset \mathcal{O}_{W, \xi}$ so that the center of the monoidal transformation is locally defined by $\left\langle x_{1}, \ldots, x_{s}\right\rangle$. Now consider an affine neighborhood $U$ of $\xi$ such that $x_{1}, \ldots, x_{s}$ are global sections of $\mathcal{O}_{U}$ and such that $J$ is generated by a global section, say $f$. For simplicity we may assume that $U=W$. The scheme $W_{1}$ is defined by patching the affine rings

$$
A_{i}=\mathcal{O}_{W}\left[x_{1} / x_{i}, \ldots, x_{s} / x_{i}\right], \quad i \in\{1, \ldots, s\},
$$

and $\mathcal{I}(H)=\left\langle x_{i}\right\rangle$ at $A_{i}$. Note also that

$$
\delta\left(\frac{x_{j}}{x_{i}}\right)=\frac{\delta\left(x_{j}\right)}{x_{i}}-\frac{x_{j}}{x_{i}} \frac{\delta\left(x_{i}\right)}{x_{i}},
$$

and that

$$
\left.\mathcal{I}(H) \delta\right|_{\operatorname{Spec}\left(A_{i}\right)}=x_{i} \delta: A_{i} \rightarrow A_{i},
$$

and hence $\mathcal{I}(H) \cdot \delta$ is an invertible sheaf of derivations on $W_{1}$.

Now in $A_{i}$ consider the factorization $f=x_{i}^{b} g_{i}$, so that $\left(\operatorname{Spec}\left(A_{i}\right),\left(\left\langle g_{i}\right\rangle, b\right)\right.$, $\left.E_{i, 1}\right)$ is the restriction of $\left(W_{1},\left(J_{1}, b\right), E_{1}\right)$ to $\operatorname{Spec}\left(A_{i}\right)$. Then by 13.7 , the transformation (19.1.1) induces a transformation

$$
(W,(\delta(\langle f\rangle), b-1), E) \longleftarrow\left(W_{1},\left(\delta(\langle f\rangle)_{1}, b-1\right), E_{1}\right) .
$$

Note here that $\langle f\rangle_{1}=\left\langle g_{i}\right\rangle \subset A_{i}$, and that $x_{i} \cdot \delta$ is a derivation on $A_{i}$. Finally check that

$$
\left.\frac{\delta(f)}{x_{i}^{b-1}}=\frac{x_{i} \delta\left(x_{i}^{b} \cdot g_{i}\right)}{x_{i}^{b}}=\frac{x_{i} \delta\left(x_{i}^{b}\right)}{x_{i}^{b}} g_{i}+x_{i}^{b} \frac{\left(x_{i} \delta\right)\left(g_{i}\right)}{x_{i}^{b}}\right)=b \cdot \delta\left(x_{i}\right) \cdot g_{i}+\left(x_{i} \delta\right)\left(g_{i}\right) .
$$

Using this formula, and the fact that $\delta: \mathcal{O}_{W} \rightarrow \mathcal{O}_{W}$ is a derivation, we also conclude that

$$
(\delta(\langle f\rangle))_{1} \subset\left\langle g_{i}, \delta(f) / x_{i}^{b-1}\right\rangle .
$$

The formula also shows that $\left\langle g_{i}, \delta(f) / x_{i}^{b-1}\right\rangle=\left\langle g_{i}, x_{i} \delta\left(g_{i}\right)\right\rangle$; and hence, that globally:

$$
(\delta(\langle f\rangle))_{1} \subset \Delta\left(\langle f\rangle_{1}\right),
$$

and now by 13.7 again we have that

$$
\operatorname{Sing}\left(\langle f\rangle_{1}, b\right) \cap \operatorname{Sing}\left((\delta(\langle f\rangle))_{1}, b-1\right)=\operatorname{Sing}\left(\langle f\rangle_{1}, b\right) .
$$

Our argument also shows that this equality is stable by any sequence of transformations. 
Corollary 19.2. For any basic object $(W,(J, b), E)$ we have that:

1) $(W,(J, b), E) \subset(W,(\Delta(J), b-1), E)$ (i.e. $(W,(J, b), E) \cap(W,(\Delta(J)$, $b-1), E)=(W,(J, b), E))$.

2) Whenever

$$
(W,(\Delta(J), b-1), E) \longleftarrow\left(W_{1},\left(\Delta(J)_{1}, b-1\right), E_{1}\right)
$$

is defined in terms of

$$
(W,(J, b), E) \longleftarrow\left(W_{1},\left(J_{1}, b\right), E_{1}\right)
$$

(in the sense of (1)), then:

$$
\Delta(J)_{1} \subset \Delta\left(J_{1}\right) .
$$

Proof: By 13.7 we have that $\operatorname{Sing}(J, b) \subset \operatorname{Sing}(\Delta(J), b-1)$, which proves (1). To prove (2), note that if

$$
(W,(J, b), E) \longleftarrow\left(W_{1},\left(J_{1}, b\right), E_{1}\right)
$$

is a projection (as in 3.12), then it is simple to check that $\Delta(J)_{1}=\Delta\left(J_{1}\right)$. Now assume that

$$
(W,(J, b), E) \longleftarrow\left(W_{1},\left(J_{1}, b\right), E_{1}\right)
$$

is a permissible transformation (as in 2.3). If $J$ is a principal sheaf of ideals, then the proof follows directly from the proof of Lemma 19.1. If $J$ is not principal, there is an open covering of $W,\left\{U_{i}\right\}_{i=1, \ldots, k}$, such that $\left.J\right|_{U_{i}}=$ $\left\langle f_{i, 1}, \ldots, f_{i, s_{i}}\right\rangle$. Then

$$
\left.(W,(J, b), E)\right|_{U_{i}}=\cap\left(W,\left(\left\langle f_{i_{j}}\right\rangle, b\right), E\right),
$$

and the statement reduces to the principal case.

Corollary 19.3. Let $(W,(J, b), E)$ be a basic object, and assume that there is a closed smooth hypersurface $\widetilde{W} \subset W$ such that

$$
\mathcal{I}(\widetilde{W}) \subset \Delta^{b-1}(J) .
$$

Then

$$
(W,(J, b), E) \subset(W,(\mathcal{I}(\widetilde{W}), 1), E) .
$$

Proof: By applying Corollary 19.2 several times, we have that

$$
(W,(J, b), E) \subset\left(W,\left(\Delta^{b-1}(J), 1\right), E\right) .
$$

Now the proof follows from the fact that $\mathcal{I}(\widetilde{W}) \subset \Delta^{b-1}(J)$ and the observation in Example 12.3. 
Corollary 19.4. Let $\mathcal{B}=(W,(J, b), E)$ be a basic object which is within the case $\operatorname{ord}_{B} \cong 1$. Then locally at any point $\xi \in \operatorname{Sing}(J, b)$, there is a smooth hypersurface $\widetilde{W}$, such that, identifying $W$ with a suitable neighborhood, $\widetilde{W}$ is closed in $W$, and

$$
(W,(J, b), E) \subset(W,(\mathcal{I}(\widetilde{W}), 1), E) .
$$

Proof: Since $\mathcal{B}$ is within the case $\cong 1$, by Remark 13.9 (d) the order of $\Delta^{b-1}(J)$ is at most one at points of $W$. So, locally at each point $\xi \in$ $V\left(\Delta^{b-1}(J)\right)=\operatorname{Sing}(J, b)$, there is an element $h_{\xi} \in \Delta^{b-1}(J)_{\xi}$ which defines a smooth hypersurface $\widetilde{W}$ at a suitable open neighborhood of $\xi$. Now the result follows from Corollary 19.3.

\section{- The restriction of basic objects to smooth hypersurfaces}

Lemma 19.5. Let $(W,(J, b), E)$ be a basic object, and let $Z \subset W$ be a closed smooth subscheme. Assume that $Z$ and $E$ are as in Definition 13.10. If $\left(\mathrm{JO}_{Z}\right)_{\xi} \neq 0$ for any $\xi \in Z$, then there is an inclusion of basic objects:

$$
(W,(J, b), E) \cap(W,(\mathcal{I}(Z), 1), E) \subset\left(Z,\left(J \mathcal{O}_{Z}, b\right), E \cap Z\right) .
$$

Proof: Set $(W,(J, b), E)=\left(W_{0},\left(J_{0}, b\right), E_{0}\right)$ and $Z_{0}=Z$. The inclusion

$$
\operatorname{Sing}\left(J_{0}, b\right) \cap \operatorname{Sing}\left(\mathcal{I}\left(Z_{0}\right), 1\right) \subset \operatorname{Sing}\left(\left(J_{0} \mathcal{O}_{Z_{0}}\right), b\right)
$$

is clear, and it is clear also that such relation will hold after a transformation as in 3.12. So we consider a sequence of transformation of pairs,

$$
\left(W_{0}, E_{0}\right) \longleftarrow\left(W_{1}, E_{1}\right) \longleftarrow \ldots \longleftarrow\left(W_{k}, E_{k}\right),
$$

with centers $C_{i} \subset W_{i}, i=0, \ldots, k-1$. Assume that this sequence defines sequences of transformations of basic objects,

$$
\begin{gathered}
\left(W_{0},\left(J_{0}, b\right), E_{0}\right) \longleftarrow\left(W_{1},\left(J_{1}, b\right), E_{1}\right) \longleftarrow \ldots \longleftarrow\left(W_{k},\left(J_{k}, b\right), E_{k}\right), \\
\left(W_{0},\left(\mathcal{I}\left(Z_{0}\right), 1\right), E_{0}\right) \longleftarrow\left(W_{1},\left(\mathcal{I}\left(Z_{1}\right), 1\right), E_{1}\right) \longleftarrow \ldots \\
\ldots \longleftarrow\left(W_{k},\left(\mathcal{I}\left(Z_{k}\right), 1\right), E_{k}\right),
\end{gathered}
$$

$$
\begin{aligned}
\left(Z_{0},\left(J_{0} \mathcal{O}_{Z_{0}}, b\right), E_{0} \cap Z_{0}\right) \longleftarrow & \left(Z_{1},\left(\left(J_{0} \mathcal{O}_{Z_{0}}\right)_{1}, b\right), E_{1} \cap Z_{1}\right) \longleftarrow \ldots \\
& \ldots \longleftarrow\left(Z_{k},\left(\left(J_{0} \mathcal{O}_{Z_{0}}\right)_{k}, b\right), E_{k} \cap Z_{k}\right),
\end{aligned}
$$


and assume, by induction, that for $i=0, \ldots, k-1$,

$$
\left(C_{i} \subset\right) \operatorname{Sing}\left(J_{i}, b\right) \cap \operatorname{Sing}\left(\mathcal{I}\left(Z_{i}\right), 1\right) \subset \operatorname{Sing}\left(\left(J_{0} \mathcal{O}_{Z_{0}}\right)_{i}, b\right) .
$$

We want to prove that

$$
\operatorname{Sing}\left(J_{k}, b\right) \cap \operatorname{Sing}\left(\mathcal{I}\left(Z_{k}\right), 1\right) \subset \operatorname{Sing}\left(\left(J_{0} \mathcal{O}_{Z_{0}}\right)_{k}, b\right) .
$$

Note that the first transformation of basic objects of sequence (19.5.3) is

$$
\left(Z_{0},\left(J_{0} \mathcal{O}_{Z_{0}}, b\right), E_{0} \cap Z_{0}\right) \longleftarrow\left(Z_{1},\left(J_{1} \mathcal{O}_{Z_{1}}, b\right), E_{1} \cap Z_{1}\right),
$$

since $\left(J_{0} \mathcal{O}_{Z_{0}}\right)_{1}=J_{1} \mathcal{O}_{Z_{1}}$. Now we have

$$
\operatorname{Sing}\left(J_{1}, b\right) \cap \operatorname{Sing}\left(\mathcal{I}\left(Z_{1}\right), 1\right) \subset \operatorname{Sing}\left(J_{1} \mathcal{O}_{Z_{1}}, b\right)=\operatorname{Sing}\left(\left(J_{0} \mathcal{O}_{Z_{0}}\right)_{1}, b\right),
$$

where the first inclusion is clear, and hence inclusion (19.5.4), and the lemma follow by induction on $k$.

Remark 19.6. Let $Z \subset W$ be a smooth closed subscheme, let $\xi \in Z$ be a closed point, and let

$$
\left\{z_{1}, \ldots, z_{r}, x_{1}, \ldots, x_{n}\right\}
$$

be a regular system of parameters in $\mathcal{O}_{W, \xi}$ such that $\mathcal{I}(Z)_{\xi}=\left(z_{1}, \ldots, z_{r}\right)$. Consider the isomorphisms

$$
\hat{\mathcal{O}}_{W, \xi} \cong k(\xi)\left[\left[z_{1}, \ldots, z_{r}, x_{1}, \ldots, x_{n}\right]\right], \quad \hat{\mathcal{O}}_{Z, \xi} \cong k(\xi)\left[\left[x_{1}, \ldots, x_{n}\right]\right],
$$

where the right sides are the rings of formal series. Given $f \in \mathcal{O}_{W, \xi}$, let $\hat{f}$ denote the image in $\hat{\mathcal{O}}_{W, \xi}$. Assume that

$$
\hat{f}=\sum_{i_{1}, \ldots, i_{r}=0}^{\infty} a_{i_{1}, \ldots, i_{r}} z_{1}^{i_{1}} \cdots z_{r}^{i_{r}},
$$

where each $a_{i_{1}, \ldots, i_{r}} \in k(\xi)\left[\left[x_{1}, \ldots, x_{n}\right]\right]$. Note that

$$
\left(i_{1} ! \cdots i_{r} !\right) a_{i_{1}, \ldots, i_{r}}=\varphi\left(\frac{\partial^{i_{1}+\cdots+i_{r}} f}{\partial z_{1}^{i_{1}} \cdots \partial z_{r}^{i_{r}}}\right)
$$

where $\varphi: k(\xi)\left[\left[z_{1}, \ldots, z_{r}, x_{1}, \ldots, x_{n}\right]\right] \rightarrow k(\xi)\left[\left[x_{1}, \ldots, x_{n}\right]\right]$ is the quotient map induced by the inclusion $Z \subset W$ at $\xi$. Note also that, for a fixed integer $b$,

$$
\nu_{\xi}(f) \geq b \Longleftrightarrow \nu_{\xi}\left(a_{i_{1}, \ldots, i_{r}}\right) \geq b-\left(i_{1}+\cdots+i_{r}\right),
$$

for all $i_{1}, \ldots, i_{r}$ with $0 \leq i_{1}+\cdots+i_{r}<b$ (here the left hand side is the order at $\mathcal{O}_{W, \xi}$, and the right hand side is the order at $\left.\mathcal{O}_{Z, \xi}\right)$. 
Proposition 19.7. Let $Z \subset W$ be a closed and smooth subscheme, and let $(W,(J, b), E)$ be a basic object. Assume that $\{E, Z\}$ have normal crossings. Then with the same notation as in Definition 13.10, if $\operatorname{Coeff}_{Z}(J)_{\xi} \neq 0$ for any $\xi \in Z$, there is an equivalence of basic objects,

$$
\left(Z,\left(\operatorname{Coeff}_{Z}(J), b !\right), E \cap Z\right) \cong(W,(J, b), E) \cap(W,(\mathcal{I}(Z), 1), E) .
$$

Proof: Set $\left(W_{0},\left(J_{0}, b\right), E_{0}\right)=(W,(J, b), E)$ and let $Z_{0}=Z$. We will restrict attention to transformations as in 3.6 and leave the case of 3.12 to the reader. Consider a sequence of transformation of pairs:

$$
\left(W_{0}, E_{0}\right) \longleftarrow\left(W_{1}, E_{1}\right) \longleftarrow \ldots \longleftarrow\left(W_{k}, E_{k}\right)
$$

with centers $C_{i} \subset W_{i}, i=0, \ldots, k-1$. Assume that this sequence defines the following three sequences of transformations:

$$
\begin{gathered}
\left(W_{0},\left(J_{0}, b\right), E_{0}\right) \longleftarrow\left(W_{1},\left(J_{1}, b\right), E_{1}\right) \longleftarrow \ldots \longleftarrow\left(W_{k},\left(J_{k}, b\right), E_{k}\right), \\
\left(W_{0},\left(\mathcal{I}\left(Z_{0}\right), 1\right), E_{0}\right) \longleftarrow\left(W_{1},\left(\mathcal{I}\left(\left(Z_{0}\right)_{1}\right), 1\right), E_{1}\right) \longleftarrow \ldots \\
\ldots \longleftarrow\left(W_{k},\left(\mathcal{I}\left(\left(Z_{0}\right)_{k}\right), 1\right), E_{k}\right), \\
\left(Z_{0},\left(\operatorname{Coeff}_{Z_{0}}\left(J_{0}\right), b !\right), E_{0} \cap Z_{0}\right) \longleftarrow\left(Z_{1},\left(\left(\operatorname{Coeff}_{Z_{0}}\left(J_{0}\right)\right)_{1}, b !\right), E_{1} \cap Z_{1}\right) \longleftarrow \ldots \\
\ldots \longleftarrow\left(Z_{k},\left(\left(\operatorname{Coeff}_{Z_{0}}\left(J_{0}\right)\right)_{k}, b !\right), E_{k} \cap Z_{k}\right) .
\end{gathered}
$$

Assume now, by induction on $k$, that for $i=0, \ldots, k-1$,

$$
\left(C_{i} \subset\right) \operatorname{Sing}\left(J_{i}, b\right) \cap Z_{i}=\operatorname{Sing}\left(\left(\operatorname{Coeff}_{Z_{0}}\left(J_{0}\right)\right)_{i}, b !\right),
$$

and recall that $\mathcal{I}\left(\left(Z_{0}\right)_{k}\right)=\mathcal{I}\left(Z_{k}\right)$, where $Z_{k}$ is the strict transform of $Z_{0}$ (see Remark $3.9(2))$, and of course $\operatorname{Sing}\left(\mathcal{I}\left(Z_{k}\right), 1\right)=Z_{k}$. We will prove that

$$
\operatorname{Sing}\left(J_{k}, b\right) \cap Z_{k}=\operatorname{Sing}\left(\left(\operatorname{Coeff}_{Z_{0}}\left(J_{0}\right)\right)_{k}, b !\right) .
$$

Consider the basic objects $\left(W_{0},\left(\Delta^{j}\left(J_{0}\right), b-j\right), E_{0}\right)$ and $\left(Z_{0},\left(\Delta^{j}\left(J_{0}\right) \mathcal{O}_{Z_{0}}, b-j\right)\right.$, $\left.E_{0} \cap Z_{0}\right)$, for $j=0, \ldots, b-1$. From Definition 13.10 it follows that

$$
\left(Z_{0},\left(\operatorname{Coeff}_{Z_{0}}\left(J_{0}\right), b !\right), E_{0} \cap Z_{0}\right)=\bigcap_{j=0}^{b-1}\left(Z_{0},\left(\Delta^{j}\left(J_{0}\right) \mathcal{O}_{Z_{0}}, b-j\right), E_{0} \cap Z_{0}\right),
$$

(see also 12.7); and by Corollary 19.2,

$$
\left.\left.\left(W_{0},\left(J_{0}, b\right), E_{0}\right)\right)=\bigcap_{j=0}^{b-1}\left(W_{0},\left(\Delta^{j}\left(J_{0}\right), b-j\right), E_{0}\right)\right) .
$$


Finally, Lemma 19.5 asserts that

$$
\left(W_{0},\left(J_{0}, b\right), E_{0}\right) \cap\left(W_{0},\left(\mathcal{I}\left(Z_{0}\right), 1\right), E_{0}\right) \subset\left(Z_{0},\left(\operatorname{Coeff}\left(J_{0}\right), b !\right), E_{0} \cap Z_{0}\right),
$$

and hence,

$$
\operatorname{Sing}\left(J_{k}, b\right) \cap Z_{k} \subset \operatorname{Sing}\left(\left(\operatorname{Coeff}_{Z_{0}}\left(J_{0}\right)\right)_{k}, b !\right) .
$$

To prove the reverse inclusion we first prove the following claim:

Claim (k): For any closed point $\xi_{k} \in \operatorname{Sing}\left(\left(\operatorname{Coeff}_{Z_{0}}\left(J_{0}\right)\right)_{k}, b !\right) \subset Z_{k}$, there is a regular system of parameters at $O_{W_{k}, \xi_{k}},\left\{z_{k, 1}, \ldots, z_{k, r}, x_{k, 1}, \ldots, x_{k, n}\right\}$, such that

(a) $\mathcal{I}\left(Z_{k}\right)_{\xi_{k}}=\left\langle z_{k, 1}, \ldots, z_{k, r}\right\rangle$.

(b) Given an isomorphism of complete rings as in Remark 19.6,

$$
\begin{gathered}
\hat{\mathcal{O}}_{W_{k}, \xi_{r}}=R_{k} \cong k\left(\xi_{k}\right)\left[\left[z_{k, 1}, \ldots, z_{k, r}, x_{k, 1}, \ldots, x_{k, n}\right]\right], \\
\hat{\mathcal{O}}_{Z_{k}, \xi_{k}}=\bar{R}_{k} \cong k\left(\xi_{k}\right)\left[\left[x_{k, 1}, \ldots, x_{k, n}\right]\right]
\end{gathered}
$$

there is a set of generators $\left\{\hat{f}_{k}^{(\lambda)}\right\}$ of $J_{k} R_{k}$ such that

$$
\hat{f}_{k}^{(\lambda)}=\sum_{i_{1}, \ldots, i_{r}=0}^{\infty} a_{k, i_{1}, \ldots, i_{r}}^{(\lambda)} z_{k, 1}^{i_{1}} \cdots z_{k, r}^{i_{r}},
$$

as in Remark 19.6, and

$$
\left(a_{k, i_{1}, \ldots, i_{r}}^{(\lambda)}\right)^{\frac{b !}{b-\left(i_{1}+\cdots+i_{r}\right)}} \in\left(\operatorname{Coeff}_{Z_{0}}\left(J_{0}\right)\right)_{k} \bar{R}_{k}
$$

Note that Claim (k) asserts that

$$
\operatorname{Sing}\left(\left(\operatorname{Coeff}_{Z_{0}}\left(J_{0}\right)\right)_{k}, b !\right) \subset \operatorname{Sing}\left(J_{k}, b\right) \cap Z_{k} .
$$

In fact, if $\xi_{k} \in \operatorname{Sing}\left(\left(\operatorname{Coeff}_{Z_{0}}\left(J_{0}\right)\right)_{k}, b\right.$ !), then the order of $\left(\operatorname{Coeff}_{Z_{0}}\left(J_{0}\right)\right)_{k} \bar{R}_{k}$ is $b$ ! at $\bar{R}_{k}$, and hence by $19.6 .2 \xi_{k} \in \operatorname{Sing}\left(J_{k}, b\right)$.

Proof of Claim (k): Claim (0) follows from (19.6.1). Assume that Claim $(k-1)$ holds for some $k \geq 1$. Let $\xi_{k-1}$ be the image of $\xi_{k}$ in $W_{k-1}$, and let

$$
\left\{z_{k-1,1}, \ldots, z_{k-1, r}, x_{k-1,1}, \ldots, x_{k-1, n}\right\}
$$

be the regular system of parameters, at $\xi_{k-1}$, provided by Claim $(k-1)$. After a finite extension of the base field, and a linear change involving only 
the variables $x_{k-1,1}, \ldots, x_{k-1, n}$ in $R_{k-1}$, we may define a regular system of parameters

$$
\left\{z_{k, 1}, \ldots, z_{k, r}, x_{k, 1}, \ldots, x_{k, n}\right\}
$$

with

$$
z_{k, i}=\frac{z_{k-1, i}}{x_{k-1,1}}, i=1, \ldots, r, \quad x_{k, 1}=x_{k-1,1}, x_{k, i}=\frac{x_{k-1, i}}{x_{k-1,1}}, i=1, \ldots, n,
$$

and $\mathcal{I}\left(Z_{k}\right)_{\xi_{k}}=\left\langle z_{k, 1}, \ldots, z_{k, r}\right\rangle$. Set

$$
\hat{f}_{k}^{(\lambda)}=\frac{\hat{f}_{k-1}^{(\lambda)}}{x_{k-1,1}^{b}}=\sum_{i_{1}, \ldots, i_{r}=0}^{\infty} a_{k, i_{1}, \ldots, i_{r}}^{(\lambda)} z_{k, 1}^{i_{1}} \cdots z_{k, r}^{i_{r}}, \quad \text { and } \quad a_{k, i_{1}, \ldots, i_{r}}^{(\lambda)}=\frac{a_{k-1, i_{1}, \ldots, i_{r}}^{(\lambda)}}{x_{k-1,1}^{b-\left(i_{1}+\cdots+i_{r}\right)}}
$$

This settles the first part of (b). To prove that

$$
\left(a_{k, i_{1}, \ldots, i_{r}}^{(\lambda)}\right)^{\frac{b !}{b-\left(i_{1}+\cdots+i_{r}\right)}} \in\left(\operatorname{Coeff}_{Z_{0}}\left(J_{0}\right)\right)_{k} \bar{R}_{k}
$$

use first (19.6.1) to show that

$$
a_{k, i_{1}, \ldots, i_{r}}^{(\lambda)} \in \Delta^{i_{1}+\cdots+i_{r}}\left(J_{k}\right) \mathcal{O}_{Z_{k}, \xi_{k}},
$$

and then Definition 13.10.1.

19.8. Proof of Proposition 13.3: It follows from Propositions 19.4 and 19.7.

\section{The monomial case}

In this section we will consider basic objects with the property that the function w-ord is equal to zero at every point. For this special case we define a resolution quite easily by means of an upper-semi-continuous function, without making any induction on the dimension of the basic object. We will see that the resolution, in this case, is purely combinatorial.

Definition 20.1. Let $(W,(J, b), E)$ be a basic object with $E=\left\{H_{1}, \ldots, H_{r}\right\}$. We say the the basic object is monomial if for any point $\xi \in \operatorname{Sing}(J, b)$ we have that

$$
J_{\xi}=\mathcal{I}\left(H_{1}\right)_{\xi}^{a_{1}(\xi)} \mathcal{I}\left(H_{2}\right)_{\xi}^{a_{2}(\xi)} \cdots \mathcal{I}\left(H_{r}\right)_{\xi}^{a_{r}(\xi)},
$$

where $a_{i}: H_{i} \cap \operatorname{Sing}(J, b) \longrightarrow \mathbb{Z}$ is a locally constant function.

Note that, for a monomial basic object, the closed set $\operatorname{Sing}(J, b)$ is the union of some of the irreducible components of intersections of the hypersurfaces $H_{i}$. In fact, consider the intersection $H_{i_{1}} \cap \cdots \cap H_{i_{p}}$ and let $C$ be an irreducible component. Then the functions $a_{i_{1}}, \ldots, a_{i_{p}}$ are constant on $C$ and $C$ is included in $\operatorname{Sing}(J, b)$ if and only $a_{i_{1}}+\cdots+a_{i_{p}} \geq b$ along $C$. 
Definition 20.2. Let $(W,(J, b), E)$ be a monomial basic object. With the notation of Definition 20.1 define the function:

$$
\begin{gathered}
h: \operatorname{Sing}(J, b) \longrightarrow \Gamma=\mathbb{Z} \times \mathbb{Q} \times \mathbb{Z}^{\mathbb{N}} \\
h(\xi)=(-p(\xi), \omega(\xi), \ell(\xi)) .
\end{gathered}
$$

If $\xi \in \operatorname{Sing}(J, b)$ define,

$$
\begin{gathered}
p(\xi)=\min \left\{q \mid \exists i_{1}, \ldots, i_{q}, \begin{array}{l}
a_{i_{1}}(\xi)+\cdots+a_{i_{q}}(\xi) \geq b \\
\xi \in H_{i_{1}} \cap \cdots \cap H_{i_{q}},
\end{array}\right\} \\
\omega(\xi)=\max \left\{\frac{a_{i_{1}}(\xi)+\cdots+a_{i_{q}}(\xi)}{b} \mid\right. \\
\left.q=p(\xi), a_{i_{1}}(\xi)+\cdots+a_{i_{q}}(\xi) \geq b, \xi \in H_{i_{1}} \cap \cdots \cap H_{i_{q}}\right\}
\end{gathered}
$$

and

$$
\begin{aligned}
\ell(\xi) & =\max \left\{\left(i_{1}, \ldots, i_{q}, 0, \ldots\right) \mid\right. \\
q & \left.=p(\xi), \frac{a_{i_{1}}(\xi)+\cdots+a_{i_{q}}(\xi)}{b}=\omega(\xi), \xi \in H_{i_{1}} \cap \cdots \cap H_{i_{q}}\right\} .
\end{aligned}
$$

In the last formula we consider the lexicographical order in $\mathbb{Z}^{\mathbb{N}}$, and the convention that $i_{1}<i_{2}<\cdots<i_{q}$.

Fix a point $\xi \in \operatorname{Sing}(J, b)$ and let $C_{1}, \ldots, C_{s}$ be the irreducible components of $\operatorname{Sing}(J, b)$ at $\xi$. The first coordinate of $h(\xi),-p(\xi)$, will indicate the minimal codimension of $C_{1}, \ldots, C_{s}$. Denote by $C_{1}^{\prime}, \ldots, C_{s^{\prime}}^{\prime}$ the components with minimum codimension $p(\xi)$. The second coordinate of $h(\xi)$ is $\omega(\xi)=b^{\prime} / b$ where $b^{\prime}$ is the maximum of the order of $J$ along the sets $C_{1}^{\prime}, \ldots, C_{s^{\prime}}^{\prime}$. Denote by $C_{1}^{\prime \prime}, \ldots, C_{s^{\prime \prime}}^{\prime \prime}$ the components with maximum order. Now the last coordinate of $h(\xi), \ell(\xi)$, corresponds to $C_{j}^{\prime \prime}$ for some index $j$.

So far, fixed a point $\xi$, with $p(\xi)$ we have selected the irreducible components of $\operatorname{Sing}(J, b)$ at $\xi$ of highest dimension. With $\omega(\xi)$ we have select, among the previous components, those where the order of $J$ is maximum. Finally with $\ell(\xi)$ we select a unique component containing $\xi$.

20.3. Now one can check that the function $h$ is upper-semi-continuous and that the set Max $h$ is regular and a union of connected components of the regular scheme $H_{i_{1}} \cap \cdots \cap H_{i_{p_{0}}}$ if $\max h=\left(-p_{0}, w_{0},\left(i_{1}, \ldots, i_{p_{0}}, 0, \ldots\right)\right)$. It is clear that $\underline{\operatorname{Max}} h$ is a permissible center for the basic object $(W,(J, b), E)$. Let

$$
(W,(J, b), E) \stackrel{\Pi}{\longleftarrow}\left(W_{1},\left(J_{1}, b\right), E_{1}\right)
$$


be the transformation with center $\underline{\operatorname{Max}} h$, and let $E_{1}=\left\{H_{1}, \ldots, H_{r}, H_{r+1}\right\}$, where, by abuse of notation, $H_{i}$ is the strict transform of $H_{i}$, for $i=1, \ldots, r$, and $H_{r+1}$ is the exceptional divisor of the transformation $\Pi$. The basic object $\left(W_{1},\left(J_{1}, b\right), E_{1}\right)$ is also monomial, in fact for $\xi \in \operatorname{Sing}\left(J_{1}, b\right)$ we have

$$
J_{\xi}=\mathcal{I}\left(H_{1}\right)_{\xi}^{a_{1}^{\prime}(\xi)} \cdots \mathcal{I}\left(H_{r}\right)_{\xi}^{a_{r}^{\prime}(\xi)} \mathcal{I}\left(H_{r+1}\right)_{\xi}^{a_{r+1}^{\prime}(\xi)}
$$

where the functions $a_{i}^{\prime}$ are given by:

$$
\begin{aligned}
& a_{i}^{\prime}(\xi) \quad=a_{i}(\Pi(\xi)) \quad \forall \xi \in H_{i} \text { and } i=1, \ldots, r \\
& a_{r+1}^{\prime}(\xi)=a_{i_{1}}(\Pi(\xi))+\cdots+a_{i_{p_{0}}}(\Pi(\xi))-b \quad \forall \xi \in H_{r+1} .
\end{aligned}
$$

As in Definition 20.2, a function $h_{1}$ has been associated to the basic object $\left(W_{1},\left(J_{1}, b\right), E_{1}\right)$, and one can check that the maximum value has dropped:

$$
\max h>\max h_{1} .
$$

In fact, for any point $\xi \in \operatorname{Sing}\left(J_{1}, b\right)$ :

$$
\begin{array}{lll}
h_{1}(\xi)=h(\Pi(\xi)) & \text { if } \quad \Pi(\xi) \notin \underline{\operatorname{Max}} h \\
h_{1}(\xi)<h(\Pi(\xi)) & \text { if } \quad \Pi(\xi) \in \underline{\operatorname{Max}} h .
\end{array}
$$

It is not hard to check that this function $h$ defines a resolution of the basic object. To illustrate this fact and the statement in (20.3.3), consider the following example:

Example 20.4. Let $(W,(J, b), E)$ where $W$ now denotes the real analytic space $\mathbb{R}^{4}, H_{1}, \ldots, H_{4}$ are the coordinate hyperplanes, and

$$
E=\left\{H_{1}, H_{2}, H_{3}, H_{4}\right\}, \quad b=9, \quad J=\mathcal{I}\left(H_{1}\right)^{6} \mathcal{I}\left(H_{2}\right)^{4} \mathcal{I}\left(H_{3}\right)^{2} \mathcal{I}\left(H_{4}\right)^{2} .
$$

Note that the intersection $H_{1} \cap H_{2} \cap H_{3} \cap H_{4}$ is a closed point. The singular locus of the basic object is:

$$
\operatorname{Sing}(J, 9)=\left(H_{1} \cap H_{2}\right) \cup\left(H_{1} \cap H_{3} \cap H_{4}\right),
$$

and the function $h$ is given by

$$
h(\xi)= \begin{cases}\left(-2, \frac{10}{9},(1,2,0,0)\right) & \text { if } \quad \xi \in H_{1} \cap H_{2} \\ \left(-3, \frac{10}{9},(1,3,4,0)\right) & \text { if } \xi \in\left(H_{1} \cap H_{3} \cap H_{4}\right) \backslash\left(H_{1} \cap H_{2}\right),\end{cases}
$$

so that $\max h=\left(-2, \frac{10}{9},(1,2,0,0)\right)$ and $\underline{\operatorname{Max}} h=H_{1} \cap H_{2}$. Note that the two irreducible components of $\operatorname{Sing}(J, 9)$ are of different dimension and that the function $p$ takes different values along each component. Consider the transformation with center $H_{1} \cap H_{2}$ :

$$
(W,(J, 9), E) \longleftarrow\left(W_{1},\left(J_{1}, 9\right), E_{1}\right),
$$

where $E_{1}=\left\{H_{1}, H_{2}, H_{3}, H_{4}, H_{5}\right\}$ and

$$
J_{1}=\mathcal{I}\left(H_{1}\right)^{6} \mathcal{I}\left(H_{2}\right)^{4} \mathcal{I}\left(H_{3}\right)^{2} \mathcal{I}\left(H_{4}\right)^{2} \mathcal{I}\left(H_{5}\right) .
$$


452 A. Bravo, S. Encinas and O. Villamayor U.

The singular locus of the new basic object is

$$
\begin{gathered}
\operatorname{Sing}\left(J_{1}, 9\right)=\left(H_{1} \cap H_{3} \cap H_{4}\right) \cup\left(H_{1} \cap H_{3} \cap H_{5}\right) \cup\left(H_{1} \cap H_{4} \cap H_{5}\right) \cup \\
\cup\left(H_{2} \cap H_{3} \cap H_{4} \cap H_{5}\right) .
\end{gathered}
$$

The function $h_{1}$ corresponding to the basic object $\left(W_{1},\left(J_{1}, 9\right), E_{1}\right)$ is given as follows:

- If $\xi \in H_{1} \cap H_{3} \cap H_{4}$ then

$$
h_{1}(\xi)=\left(-3, \frac{10}{9},(1,3,4,0)\right) .
$$

- If $\xi \in H_{1} \cap H_{4} \cap H_{5}$ and $\xi \notin H_{1} \cap H_{3} \cap H_{4}$ then

$$
h_{1}(\xi)=\left(-3, \frac{9}{9},(1,4,5,0)\right)
$$

- If $\xi \in H_{1} \cap H_{3} \cap H_{5}$ and $\xi \notin\left(H_{1} \cap H_{3} \cap H_{4}\right) \cup\left(H_{1} \cap H_{4} \cap H_{5}\right)$ then

$$
h_{1}(\xi)=\left(-3, \frac{9}{9},(1,3,5,0)\right)
$$

- If $\xi \in H_{2} \cap H_{3} \cap H_{4} \cap H_{5}$ then

$$
h_{1}(\xi)=\left(-4, \frac{9}{9},(2,3,4,5)\right)
$$

Here $\operatorname{Sing}\left(J_{1}, 9\right)$ has four irreducible components and the function $h_{1}$ takes different values along each component. Then $\max h_{1}=\left(-3, \frac{10}{9},(1,3,4,0)\right)$ and $\underline{\operatorname{Max}} h_{1}=H_{1} \cap H_{3} \cap H_{4}$. Note that $\max h>\max h_{1}$. We construct the transformation of basic objects with center $\underline{\operatorname{Max}} h_{1}$ :

$$
\left(W_{1},\left(J_{1}, 9\right), E_{1}\right) \longleftarrow\left(W_{2},\left(J_{2}, 9\right), E_{2}\right) .
$$

Now $E_{2}=\left\{H_{1}, H_{2}, H_{3}, H_{4}, H_{5}, H_{6}\right\}$ and

$$
J_{2}=\mathcal{I}\left(H_{1}\right)^{6} \mathcal{I}\left(H_{2}\right)^{4} \mathcal{I}\left(H_{3}\right)^{2} \mathcal{I}\left(H_{4}\right)^{2} \mathcal{I}\left(H_{5}\right) \mathcal{I}\left(H_{6}\right) .
$$

The singular locus of $\left(W_{2},\left(J_{2}, 9\right), E_{2}\right)$ is

$$
\begin{aligned}
\operatorname{Sing}\left(J_{2}, 9\right)= & \left(H_{1} \cap H_{3} \cap H_{5}\right) \cup\left(H_{1} \cap H_{4} \cap H_{5}\right) \cup \\
& \cup\left(H_{1} \cap H_{3} \cap H_{6}\right) \cup\left(H_{1} \cap H_{4} \cap H_{6}\right) \cup \\
& \cup\left(H_{2} \cap H_{3} \cap H_{4} \cap H_{5}\right) \cup\left(H_{2} \cap H_{3} \cap H_{4} \cap H_{6}\right) .
\end{aligned}
$$

There are four irreducible components of dimension one and two components of dimension zero. The function $h_{2}$ takes different values along each 
component and one concludes that $\max h_{2}=\left(-3, \frac{9}{9},(1,4,6,0)\right)$ and that $\underline{\operatorname{Max}} h_{2}=H_{1} \cap H_{4} \cap H_{6}$. Consider the transformation with center $\underline{\operatorname{Max}} h_{2}$

$$
\left(W_{2},\left(J_{2}, 9\right), E_{2}\right) \longleftarrow\left(W_{3},\left(J_{3}, 9\right), E_{3}\right),
$$

where $E_{3}=\left\{H_{1}, H_{2}, H_{3}, H_{4}, H_{5}, H_{6}, H_{7}\right\}$ and

$$
J_{3}=\mathcal{I}\left(H_{1}\right)^{6} \mathcal{I}\left(H_{2}\right)^{4} \mathcal{I}\left(H_{3}\right)^{2} \mathcal{I}\left(H_{4}\right)^{2} \mathcal{I}\left(H_{5}\right) \mathcal{I}\left(H_{6}\right) .
$$

Now the singular locus is

$$
\begin{gathered}
\operatorname{Sing}\left(J_{3}, 9\right)=\left(H_{1} \cap H_{3} \cap H_{5}\right) \cup\left(H_{1} \cap H_{4} \cap H_{5}\right) \cup \\
\cup\left(H_{1} \cap H_{3} \cap H_{6}\right) \cup\left(H_{2} \cap H_{3} \cap H_{4} \cap H_{5}\right) \cup\left(H_{2} \cap H_{3} \cap H_{4} \cap H_{6}\right),
\end{gathered}
$$

and we have that $\max h_{3}=\left(-3, \frac{9}{9},(1,4,5,0)\right)$ and $\underline{\operatorname{Max}} h_{3}=H_{1} \cap H_{4} \cap H_{5}$. After five transformations of basic objects we achieve a resolution.

\section{On Hironaka's trick}

The purpose of this section is to state and prove Proposition 21.1. A consequence of this result is that the function ord introduced in 6.1 is well defined for general basic objects, and moreover, equivariant (see Remark 21.2).

Proposition 21.1. [4] Let $(\mathcal{F},(W, E))$ be a d-dimensional general basic object. Assume that there is an open covering $\left\{U_{\alpha}\right\}_{\alpha \in \Lambda}$ of $W$ as in Definition 14.1 consisting of the single open set $W$. Assume also that we have two $d$-dimensional basic objects $\left(W^{\prime},\left(B^{\prime}, d^{\prime}\right), E^{\prime}\right)$ and $\left(W^{\prime \prime},\left(B^{\prime \prime}, d^{\prime \prime}\right), E^{\prime \prime}\right)$ defining $(\mathcal{F},(W, E))$, (i.e. both basic objects describe the closed sets associated to $(\mathcal{F},(W, E)))$. If $x \in \operatorname{Sing}\left(B^{\prime}, d^{\prime}\right)=\operatorname{Sing}\left(B^{\prime \prime}, d^{\prime \prime}\right)$ then

$$
\frac{\boldsymbol{\nu}_{B^{\prime}}(x)}{d^{\prime}}=\frac{\boldsymbol{\nu}_{B^{\prime \prime}}(x)}{d^{\prime \prime}}
$$

Proof: Set $\omega^{\prime}=\boldsymbol{\nu}_{B^{\prime}}(x)$ and $\omega^{\prime \prime}=\boldsymbol{\nu}_{B^{\prime \prime}}(x)$. We shall prove the proposition by constructing a number of $x$-extendable sequences of transformations of general basic objects with suitable properties. Let

$$
(\mathcal{F},(W, E)) \stackrel{\Pi_{0}}{\longleftarrow}\left(\mathcal{F}_{0},\left(W_{0}, E_{0}\right)\right) \stackrel{\Pi_{1}}{\longleftarrow}\left(\mathcal{F}_{1},\left(W_{1}, E_{1}\right)\right) \stackrel{\Pi_{2}}{\longleftarrow} \cdots \stackrel{\Pi_{k}}{\longleftarrow}\left(\mathcal{F}_{k},\left(W_{k}, E_{k}\right)\right)
$$

be a sequence of transformations of general basic objects. Note that sequence (21.1.1) defines sequences of transformations of basic objects

$$
\begin{aligned}
\left(W^{\prime},\left(B^{\prime}, d^{\prime}\right), E^{\prime}\right) \stackrel{\Pi_{0}^{\prime}}{\longleftarrow}\left(W_{0}^{\prime},\left(B_{0}^{\prime}, d^{\prime}\right), E_{0}^{\prime}\right) \stackrel{\Pi_{1}^{\prime}}{\longleftarrow}\left(W_{1}^{\prime},\left(B_{1}^{\prime}, d^{\prime}\right), E_{1}^{\prime}\right) \stackrel{\Pi_{2}^{\prime}}{\longleftarrow} \ldots \\
\ldots \stackrel{\Pi_{k}^{\prime}}{\longleftarrow}\left(W_{k}^{\prime},\left(B_{k}^{\prime}, d^{\prime}\right), E_{k}^{\prime}\right),
\end{aligned}
$$


and

$$
\begin{array}{r}
\left(W^{\prime \prime},\left(B^{\prime \prime}, d^{\prime \prime}\right), E^{\prime \prime}\right) \stackrel{\Pi_{0}^{\prime \prime}}{\longleftarrow}\left(W_{0}^{\prime \prime},\left(B_{0}^{\prime \prime}, d^{\prime \prime}\right), E_{0}^{\prime \prime}\right) \stackrel{\Pi_{1}^{\prime \prime}}{\longleftarrow}\left(W_{1}^{\prime \prime},\left(B_{1}^{\prime \prime}, d^{\prime \prime}\right), E_{1}^{\prime \prime}\right) \stackrel{\Pi_{2}^{\prime \prime}}{\longleftarrow} \ldots \\
\\
\cdots \stackrel{\Pi_{k}^{\prime \prime}}{\longleftarrow}\left(W_{k}^{\prime \prime},\left(B_{k}^{\prime \prime}, d^{\prime \prime}\right), E_{k}^{\prime \prime}\right) .
\end{array}
$$

The first transformation $\Pi_{0}$ of (21.1.1) is a projection (as allowed in 14.1), so the first transformations of (21.1.2) and (21.1.3) are projections too. All the other transformation will be permissible transformations (as in 3.6). For each index $k$, sequence (21.1.1) is defined as follows:

1. Identify $L_{0}=\Pi_{0}^{-1}(x)$ with $\mathbb{A}_{k}^{1}$ and set $x_{0}=0 \in L_{0}$. Note that $L_{0} \subset F_{0}$, the singular locus of $\left(\mathcal{F}_{0},\left(W_{0}, E_{0}\right)\right)$.

2. Given an index $s \geq 0$, a line $L_{s} \subset F_{s}$ and a point $x_{s} \in L_{s}$, define the transformation $\Pi_{s+1}$ with center $x_{s}$. Now set:

i $L_{s+1} \subset F_{s+1}$ as the strict transform of $L_{s}$.

ii $H_{s+1}\left(\in E_{s+1}\right)$ as the exceptional locus of $\Pi_{s+1}$;

iii $x_{s+1}=H_{s+1} \cap L_{s+1}$.

Note that (1) together with (2) provides us with a rule for constructing a sequence as (21.1.1) with length $s$ for any $s \geq 1$. By construction the sequence (21.1.1) is $x$-extendable (see 6.3). Note that $L_{s} \subset F_{s}$ for any $s$, so in particular $x_{s} \in F_{s}$, and by assumption:

$$
x_{s} \in \operatorname{Sing}\left(B_{s}^{\prime}, d^{\prime}\right)=\operatorname{Sing}\left(B_{s}^{\prime \prime}, d^{\prime \prime}\right) \quad \forall s \geq 0 .
$$

There are expressions as in (15.20.2),

$$
\left(B_{s}^{\prime}\right)_{x_{s}}=\mathcal{I}\left(H_{s}^{\prime}\right)_{x_{s}}^{a_{s}^{\prime}}\left(\overline{B^{\prime}}{ }_{s}\right)_{x_{s}} \quad\left(B_{s}^{\prime \prime}\right)_{x_{s}}=\mathcal{I}\left(H_{s}^{\prime \prime}\right)_{x_{s}}^{a_{s}^{\prime \prime}}\left(\overline{B^{\prime \prime}}{ }_{s}\right)_{x_{s}} .
$$

Note that here $H_{s}^{\prime}=H_{s} \cap W_{s}^{\prime}$ and $H_{s}^{\prime \prime}=H_{s} \cap W_{s}^{\prime \prime}$. On may check, by induction on $s$, that

$$
a_{s}^{\prime}=s\left(\omega^{\prime}-d^{\prime}\right) \quad a_{s}^{\prime \prime}=s\left(\omega^{\prime \prime}-d^{\prime \prime}\right) .
$$

Since the first term of all sequences is a projection, then for $s \geq 1, \operatorname{dim}\left(W_{s}^{\prime}\right)=$ $\operatorname{dim}\left(W_{s}^{\prime \prime}\right)=d+1$. It follows that

$$
\begin{aligned}
\operatorname{dim}\left(F_{s} \cap H_{s}\right)=d & \Longleftrightarrow a_{s}^{\prime}=s\left(\omega^{\prime}-d^{\prime}\right) \geq d^{\prime} \\
& \Longleftrightarrow a_{s}^{\prime \prime}=s\left(\omega^{\prime \prime}-d^{\prime \prime}\right) \geq d^{\prime \prime} .
\end{aligned}
$$

Note that $\operatorname{dim} H_{s}^{\prime}=\operatorname{dim} H_{s}^{\prime \prime}=d$, so if $\operatorname{dim}\left(F_{s} \cap H_{s}\right)=d$ then $F_{s} \cap H_{s}=$ $H_{s}^{\prime}=H_{s}^{\prime \prime}$. Furthermore if $\operatorname{dim}\left(F_{s} \cap H_{s}\right)=d$, then $F_{s} \cap H_{s}$ is a permissible center for the general basic object. 
At this point we start a new stage of the process by choosing $F_{s} \cap H_{s}$ as a center of a transformation $\Pi_{s+1}$. It turns out that in the expressions of (21.1.4),

$$
a_{s}^{\prime}=s\left(\omega^{\prime}-d^{\prime}\right)-d^{\prime}, \quad a_{s}^{\prime \prime}=s\left(\omega^{\prime \prime}-d^{\prime \prime}\right)-d^{\prime \prime} .
$$

Fix the index $s$ and set, if possible, the center of transformations $\Pi_{s+j}$ to be $F_{s+j} \cap H_{s+j}$, for $j \geq 0$. Note that

$$
\begin{aligned}
\operatorname{dim}\left(F_{s+j} \cap H_{s+j}\right)=d & \Longleftrightarrow a_{s+j}^{\prime}=s\left(\omega^{\prime}-d^{\prime}\right)-j d^{\prime} \geq d^{\prime} \\
& \Longleftrightarrow a_{s+j}^{\prime \prime}=s\left(\omega^{\prime \prime}-d^{\prime \prime}\right)-j d^{\prime \prime} \geq d^{\prime \prime} .
\end{aligned}
$$

And we conclude that

$$
\begin{aligned}
\operatorname{dim}\left(F_{s+j} \cap H_{s+j}\right)=d \text { (in which case is a permissible center) } & \Leftrightarrow j \leq \ell_{s}^{\prime} \\
& \Leftrightarrow j \leq \ell_{s}^{\prime \prime},
\end{aligned}
$$

where

$$
\ell_{s}^{\prime}=\left\lfloor\frac{s\left(\omega^{\prime}-d^{\prime}\right)}{d^{\prime}}\right\rfloor \quad \ell_{s}^{\prime \prime}=\left\lfloor\frac{s\left(\omega^{\prime \prime}-d^{\prime \prime}\right)}{d^{\prime \prime}}\right\rfloor
$$

and $\lfloor\cdot\rfloor$ denotes the integer part.

Finally note that

$$
\begin{aligned}
& \frac{\boldsymbol{\nu}_{B^{\prime}}(x)}{d^{\prime}}=\frac{w^{\prime}}{d^{\prime}}=\lim _{s \rightarrow \infty} \frac{1}{s} \ell_{s}^{\prime}+1, \\
& \frac{\boldsymbol{\nu}_{B^{\prime \prime}}(x)}{d^{\prime \prime}}=\frac{w^{\prime \prime}}{d^{\prime \prime}}=\lim _{s \rightarrow \infty} \frac{1}{s} \ell_{s}^{\prime \prime}+1,
\end{aligned}
$$

and we have expressed the rational numbers

$$
\frac{\boldsymbol{\nu}_{B^{\prime}}(x)}{d^{\prime}} \quad \text { and } \quad \frac{\boldsymbol{\nu}_{B^{\prime \prime}}(x)}{d^{\prime \prime}}
$$

in terms of permissible sequences of the general basic object $(\mathcal{F},(W, E))$, and all sequences are $x$-extendable. Hence $\boldsymbol{\nu}_{B^{\prime}}(x) / d^{\prime}=\boldsymbol{\nu}_{B^{\prime \prime}}(x) / d^{\prime \prime}$.

Remark 21.2. It follows from Proposition 21.1 that the function ord is well defined for general basic objects, and note also that the proof of Proposition 21.1 implies the equivariance of this function: Given an isomorphism of $d$-dimensional general basic objects $\Theta:(\mathcal{F},(W, E)) \rightarrow\left(\mathcal{F}^{\prime},\left(W^{\prime}, E^{\prime}\right)\right.$ ) (see Definition 14.5) and a point $x \in \operatorname{Sing}(\mathcal{F})$, set $x^{\prime}=\Theta(x) \in \operatorname{Sing}\left(\mathcal{F}^{\prime}\right)$. By Definition 14.5 (ii), the isomorphism $\Theta$ defines a bijection between $x$-extendable sequences and $x^{\prime}$-extendable sequences, say $\alpha_{\Theta}: C_{x}(\mathcal{F}) \rightarrow C_{x^{\prime}}\left(\mathcal{F}^{\prime}\right)$. Note that the dimension $n$ is fixed and the rational number $\operatorname{ord}_{\mathcal{F}}(x)\left(\operatorname{resp} . \operatorname{ord}_{\mathcal{F}^{\prime}}\left(x^{\prime}\right)\right)$ is expressed in terms of sequences of $C_{x}(\mathcal{F})$ (resp. $C_{x^{\prime}}\left(\mathcal{F}^{\prime}\right)$ ). We conclude that $\operatorname{ord}_{\mathcal{F}}(x)=\operatorname{ord}_{\mathcal{F}^{\prime}}\left(x^{\prime}\right)$. 


\section{References}

[1] Abhyankar, S. S.: Good points of a hypersurface. Adv. in Math. 68 (1988), 87-256.

[2] Abramovich, D. and De Jong, A. J. : Smoothness, semistability and toroidal geometry. J. Algebraic Geom. 6 (1997), 789-801.

[3] Abramovich, D. And Wang, J.: Equivariant resolution of singularities in characteristic 0. Math. Res. Lett. 4 (1997), 427-433.

[4] Aroca, J. M., Hironaka, H. and Vicente, J. L.: The theory of the maximal contact. Memorias de Matemática del Instituto "Jorge Juan" 29. Instituto "Jorge Juan" de Matemáticas, Consejo Superior de Investigaciones Cientificas, Madrid, 1975.

[5] Artin, M.: Algebraic approximation of structures over complete local rings. Inst. Hautes Études Sci. Publ. Math. 36 (1969), 23-58.

[6] Bierstone, E. And Milman, P.: A simple constructive proof of canonical resolution of singularities. In Effective methods in algebraic geometry (Castiglioncello, 1990), 11-30. Progr. Math. 94. Birkhäuser, Boston, MA, 1991.

[7] Bierstone, E. And Milman, P.: Canonical desingularization in characteristic zero by blowing up the maximum strata of a local invariant. Invent. Math. 128 (1997), 207-302.

[8] Bierstone, E. And Milman, P.: Desingularization algorithms I. Role of exceptional divisors. Mosc. Math. J. 3 (2003), no. 3, 751-805.

[9] Bodnár, G., Encinas, S. And Schicho, J.: A local presentation of Varieties. Preprint.

[10] Bodnár, G. And Schicho, J.: A Computer Program for the Resolution of Singularities. In Resolution of singularities, 231-238. Progr. Math. 181. Birkhäuser, Basel, 2000.

[11] Bodnár, G. And Schicho, J.: Automated resolution of singularities for hypersurfaces. J. Symbolic Comput. 30 (2000), no. 4, 401-428.

[12] Bogomolov, F. And Pantev, T.: Weak Hironaka Theorem. Math. Res. Lett. 3 (1996), no. 3, 299-307.

[13] Bravo, A. and Villamayor U., O.: Strengthening the theorem of embedded desingularization. Math. Res. Lett. 8 (2001), 79-89.

[14] Bravo, A. and Villamayor U., O.: A Strengthening of resolution of singularities in characteristic zero. Proc. London Math. Soc. (3) 86 (2003), no. $2,327-357$.

[15] Encinas, S. And Hauser, H.: Strong Resolution of Singularities in characteristic zero. Comment. Math. Helv. 77 (2002), no. 4, 821-845.

[16] Encinas, S., Nobile, A. and Villamayor U., O.: On algorithmic equi-resolution and stratification of Hilbert schemes. Proc. London Math. Soc. (3) 86 (2003), no. 3, 607-648. 
[17] Encinas, S. and Villamayor U., O.: Good points and constructive resolution of singularities. Acta Math. 181 (1998), no. 1, 109-158.

[18] Encinas, S. and Villamayor U., O.: A course on constructive desingularization and equivariance. In Resolution of singularities, 147-227. Progr. Math. 181. Birkhäuser, Basel, 2000.

[19] Encinas, S. and Villamayor U., O.: A new Proof of Desingularization over fields of characteristic zero. Rev. Mat. Iberoamericana 19 (2003), 339-353.

[20] Giraud, J.: Sur la théorie du contact maximal. Math. Z. 137 (1974), 285-310.

[21] Grothendieck, A. And Raynaud, M.: Revêtements étales et groupe fondamental. Séminaire de Géométrie Algébrique du Bois Marie 19601961. Lecture Notes in Mathematics 224. Springer-Verlag, Berlin-New York, 1971.

[22] Hauser, H.: The Hironaka theorem on resolution of singularities. Bull. Amer. Math. Soc. (N.S.) 40 (2003), no. 3, 323-403.

[23] Hironaka, H.: Resolution of singularities of an algebraic variety over a field of characteristic zero I, II. Ann. of Math. (2) 79 (1964), 109-203 and 205-326.

[24] Hironaka, H.: Idealistic exponent of a singularity. In Algebraic geometry (J. J. Sylvester Sympos., Johns Hopkins Univ., Baltimore 1976), 52-125. Johns Hopkins Univ. Press, Baltimore, 1977.

[25] Lazarsfeld, R.: Positivity in algebraic geometry. I. Classical setting: line bundles and linear series. Ergeb. Math. Grenzgeb. (3) 48. Springer-Verlag, Berlin, 2004. Positivity in algebraic geometry. II. Positivity for vector bundles, and multiplier ideals. Ergeb. Math. Grenzgeb. (3) 49. Springer-Verlag, Berlin, 2004.

[26] Lipman, J.: Introduction to resolution of singularities. In Algebraic geometry, Humboldt State Univ., Arcata 1974), 187-230. Proc. Sympos. Pure Math. 29. Amer. Math. Soc., Providence, R.I., 1975.

[27] Lipman, J.: Equisingularity and simultaneous resolution of singularities. In Resolution of singularities (Obergurgl, 1997), 485-505. Progr. Math. 181. Birkhäuser, Basel, 2000.

[28] Matsuki, K.: Notes on the inductive algorithm of resolution of singularities by S. Encinas and O. Villamayor. Preprint. arXiv:math.math. AG/0103120.

[29] Matsumura, H.: Commutative algebra. Mathematics Lecture Note Series 56. Benjamin/Cummings Publishing Company, Inc., 2nd edition, 1980.

[30] МoH, T. T.: Quasi-canonical uniformization of hypersurface singularities of characteristic zero. Comm. Algebra 20 (1992), 3207-3249.

[31] Mumford, D.: Geometric invariant theory. Ergebnisse der Mathematik und ihrer Grenzgebiete 34. Springer-Verlag, Berlin-New York, 1965. 
[32] ODA, T.: Infinitely very near singular points. In Complex analytic singularities, 363-404. Adv. Stud. Pure Math. 8. North-Holland, Amsterdam, 1987.

[33] Stromme, S. A.: Elementary introduction to representable functors and Hilbert Schemes. In Parameter Spaces (Warsaw, 1994), 179-198. Banach Center Publ. 36. Polish Acad. Sci., Warsaw, 1996.

[34] Villamayor U., O.: Constructiveness of Hironaka's resolution. Ann. Sci. École Norm. Sup. (4) 22 (1989), no. 1, 1-32.

[35] Villamayor U., O.: Patching local uniformizations. Ann. Sci. École Norm. Sup. (4) 25 (1992), 629-677.

[36] Villamayor U., O.: Introduction to the algorithm of resolution. In Algebraic geometry and singularities (La Rábida, 1991), 123-154. Progr. Math. 134. Birkhäuser, Basel, 1996.

Recibido: 24 de abril de 2002

Revisado: 27 de marzo de 2003

Ana María Bravo

Departamento de Matemáticas

Facultad de Ciencias

Universidad Autónoma de Madrid

Canto Blanco 28049 Madrid, Spain

ana.bravo@uam.es

Santiago Encinas

Departamento de Matemática Aplicada

ETS Arquitectura

Universidad de Valladolid

Avda. Salamanca s/n

47014-Valladolid, Spain

sencinas@maf.uva.es

Orlando Villamayor U.

Departamento de Matemáticas

Facultad de Ciencias

Universidad Autónoma de Madrid

Canto Blanco 28049 Madrid, Spain

villamayor@uam.es

All the authors were partially supported by PB96-0065. The first author was also partially supported by a Rackham Fellowship during the summer in 2001. 